TRANSACTIONS OF THE

AMERICAN MATHEMATICAL SOCIETY

Volume 358, Number 9, September 2006, Pages 3883-3936

S 0002-9947(06)04056-6

Article electronically published on April 17, 2006

\title{
CALCULUS ON THE SIERPINSKI GASKET II: POINT SINGULARITIES, EIGENFUNCTIONS, AND NORMAL DERIVATIVES OF THE HEAT KERNEL
}

\author{
NITSAN BEN-GAL, ABBY SHAW-KRAUSS, ROBERT S. STRICHARTZ, \\ AND CLINT YOUNG
}

\begin{abstract}
This paper continues the study of fundamental properties of elementary functions on the Sierpinski gasket (SG) related to the Laplacian defined by Kigami: harmonic functions, multiharmonic functions, and eigenfunctions of the Laplacian. We describe the possible point singularities of such functions, and we use the description at certain periodic points to motivate the definition of local derivatives at these points. We study the global behavior of eigenfunctions on all generic infinite blow-ups of SG, and construct eigenfunctions that decay at infinity in certain directions. We study the asymptotic behavior of normal derivatives of Dirichlet eigenfunctions at boundary points, and give experimental evidence for the behavior of the normal derivatives of the heat kernel at boundary points.
\end{abstract}

\section{INTRODUCTION}

In this paper we continue the study of fundamental properties of elementary functions on the Sierpinski gasket (SG). In [NSTY], which we will refer to as part I, the growth properties of monomials, analogous to $x^{n} / n$ ! on the unit interval, were described, and this information was used to give a theory of power series and analytic functions on SG. Recall that SG is the unique nonempty compact set in the plane satisfying

$$
K=\bigcup_{i=0}^{2} F_{i} K
$$

where $F_{i} x=\frac{1}{2} x+\frac{1}{2} q_{i}$ and $q_{0}, q_{1}, q_{2}$ are the vertices of an equilateral triangle considered the boundary of $K$ with a self-similar and symmetric energy form $\mathcal{E}(u, v)$, measure $\mu$ and Laplacian $\Delta$ as described in [Ki1, Ki2]. (See also [S2], S7].)

Received by the editors June 8, 2004.

2000 Mathematics Subject Classification. Primary 28A80.

The first, second and fourth authors' research was supported by the National Science Foundation through the Research Experiences for Undergraduates (REU) Program at Cornell University.

The third author's research was supported in part by the National Science Foundation, grant DMS-0140194. 
The self-similar properties are

$$
\begin{gathered}
\mathcal{E}(u, v)=\frac{5}{3} \sum_{i=0}^{2} \mathcal{E}\left(u \circ F_{i}, v \circ F_{i}\right), \\
\mu=\frac{1}{3} \sum_{i=0}^{2} \mu \circ F_{i}^{-1},
\end{gathered}
$$

and

$$
\Delta\left(u \circ F_{i}\right)=\frac{1}{5}(\Delta u) \circ F_{i},
$$

or more generally

$$
\begin{gathered}
\mathcal{E}(u, v)=\left(\frac{5}{3}\right)^{m} \sum_{|w|=m} \mathcal{E}\left(u \circ F_{w}, v \circ F_{w}\right), \\
\mu=\frac{1}{3^{m}} \sum_{|w|=m} \mu \circ F_{w}^{-1},
\end{gathered}
$$

and

$$
\Delta\left(u \circ F_{w}\right)=\frac{1}{5^{m}}(\Delta u) \circ F_{w},
$$

where $w=\left(w_{1}, w_{2}, \ldots, w_{m}\right)$ denotes a word of length $|w|=m$ in the digits $(0,1,2)$ and $F_{w}=F_{w_{1}} F_{w_{2}} \cdots F_{w_{m}}$. The Laplacian may be defined by the weak formulation $u \in \operatorname{dom} \Delta$ and $\Delta u=f$ if $u \in \operatorname{dom} \mathcal{E}, f$ is continuous, and

$$
-\mathcal{E}(u, v)=\int f v d \mu \text { for all } v \in \operatorname{dom}_{0} \mathcal{E},
$$

where the subscript 0 indicates that the function vanishes on the boundary. There are also local derivatives defined at the boundary points. At $q_{0}$, the normal derivative is defined by

$$
\partial_{n} u\left(q_{0}\right)=\lim _{m \rightarrow \infty}\left(\frac{5}{3}\right)^{m}\left[2 u\left(q_{0}\right)-u\left(F_{0}^{m} q_{1}\right)-u\left(F_{0}^{m} q_{2}\right)\right]
$$

and the tangential derivative by

$$
\partial_{T} u\left(q_{0}\right)=\lim _{m \rightarrow \infty} 5^{m}\left[u\left(F_{0}^{m} q_{1}\right)-u\left(F_{0}^{m} q_{2}\right)\right],
$$

with similar definitions at $q_{1}$ and $q_{2}$ by cyclic permutation of the indices. These local derivatives exist if $u \in \operatorname{dom} \Delta$. Then (1.8) extends to the Gauss-Green formula

$$
\int v \Delta u d \mu=-\mathcal{E}(u, v)+\sum_{i=0}^{2} v\left(q_{i}\right) \partial_{n} u\left(q_{i}\right)
$$

for all $v \in \operatorname{dom} \mathcal{E}, u \in \operatorname{dom} \Delta$.

The space of harmonic functions on $K$, solutions of $\Delta u=0$, is 3-dimensional, each harmonic function being determined by its 3 boundary values. More generally, a function is called multiharmonic if $\Delta^{k+1} u=0$ for some $k \geq 0$. These are the analogs of polynomials on the unit interval. In part I, a family of multiharmonic functions, analogous to the monomials $x^{n} / n$ ! on the unit interval, was described and 
studied. Choose a boundary point, say $q_{0}$. Then the functions $P_{j k}^{(0)}$ for $k=1,2,3$ and $j \geq 0$ satisfy $\Delta^{j+1} P_{j k}^{(0)}=0$ and are characterized by the conditions

$$
\left\{\begin{array}{l}
\Delta^{m} P_{j k}^{(0)}\left(q_{0}\right)=\delta_{m j} \delta_{k 1}, \\
\partial_{n} \Delta^{m} P_{j k}^{(0)}\left(q_{0}\right)=\delta_{m j} \delta_{k 2}, \\
\partial_{T} \Delta^{m} P_{j k}^{(0)}\left(q_{0}\right)=\delta_{m j} \delta_{k 3} .
\end{array}\right.
$$

These functions are related by the identity

$$
\Delta P_{(j+1) k}^{(0)}=P_{j k}^{(0)} .
$$

If $R_{0}$ denotes the reflection symmetry of $K$ that fixes $q_{0}$ and interchanges $q_{1}$ and $q_{2}$, then $P_{j 1}^{(0)}$ and $P_{j 2}^{(0)}$ are symmetric while $P_{j 3}^{(0)}$ is skew-symmetric with respect to $R_{0}$. The decay properties of these functions near $q_{0}$ are described by the following scaling identities:

$$
\left\{\begin{array}{l}
P_{j 1}^{(0)}\left(F_{0}^{m} x\right)=5^{-j m} P_{j 1}^{(0)}(x), \\
P_{j 2}^{(0)}\left(F_{0}^{m} x\right)=\left(\frac{3}{5}\right)^{m} 5^{-j m} P_{j 2}^{(0)}(x), \\
P_{j 3}^{(0)}\left(F_{0}^{m} x\right)=5^{-(j+1) m} P_{j 3}^{(0)}(x) .
\end{array}\right.
$$

In section 4 of this paper, we will see that if we consider solutions of $\Delta^{j+1} u=0$ in $K \backslash q_{0}$ with possible singularity at $q_{0}$, then we add another family $P_{j 4}^{(0)}$. These functions are skew-symmetric with respect to $R_{0}$ and satisfy (1.13) and the scaling identity

$$
P_{j 4}^{(0)}\left(F_{0}^{m} x\right)=3^{m} \cdot 5^{-j m} P_{j 4}^{(0)}(x) .
$$

We observe from (1.15) that while $P_{04}^{(0)}$ has a pole at $q_{0}$, all the other $P_{j 4}^{(0)}$ functions are continuous, and in fact vanish at $q_{0}$. This is reminiscent of the terms in expansions that hold for solutions of ordinary differential equations in a neighborhood of a regular singular point. We also have experimental evidence for the asymptotic behavior of $P_{j 4}^{(0)}$ as $j \rightarrow \infty$, namely that $\lim _{j \rightarrow \infty}\left(-5 \lambda_{0}^{(5)}\right)^{j} P_{j 4}^{(0)}$ exists and equals an eigenfunction with eigenvalue $5 \lambda_{0}^{(5)} \approx 279.4291373 \ldots$. (The notation is from GRS].) This is similar to the asymptotic behavior of $P_{j 2}^{(0)}$ established in part I, while $P_{j 1}^{(0)}$ and $P_{j 3}^{(0)}$ have superexponential decay in $j$.

We are also interested in functions with point singularities at other types of points. We begin by considering just harmonic functions. In section 2 , we show that if the singularity occurs at a junction point the space of harmonic functions is 7-dimensional, while at a generic point it is 6 -dimensional. We are also able to localize singularities and describe the space of harmonic functions with a finite number of singularities.

In section 3, we specialize to the case of harmonic functions with a singularity at a periodic point $z$, meaning that $z$ is the fixed-point of $F_{w}$ for some finite word $w$ of length $m$. (So $z$ has the address $w, w, w, \ldots$ ) We describe a specific basis $\left\{P_{0 k}^{(z)}\right\}_{k=1,2, \ldots, 6}$ for this space, characterized by the scaling identities

$$
P_{0 k}^{(z)} \circ\left(F_{w}\right)^{n}=\sigma_{k}^{n} P_{0 k}^{(z)}
$$

for certain constants $\sigma_{k}$. When $k=1,2,3$, these functions are globally harmonic (have removable singularities) with typically $1=\sigma_{1}>\sigma_{2}>\sigma_{3}$. (There is at least 
one exception, when $w=(1,2,3)$, where $\sigma_{2}$ and $\sigma_{3}$ are complex conjugate pairs with $\left|\sigma_{2}\right|=\left|\sigma_{3}\right|<1$.) The remaining functions $P_{0 k}^{(z)}$ for $k=4,5,6$ have true singularities at $z$, although $P_{04}^{(z)}$ is continuous since $\sigma_{4}=\left(\frac{3}{5}\right)^{m}<1$, while $P_{05}^{(z)}$ and $P_{06}^{(z)}$ have poles since $1<\sigma_{5}<\sigma_{6}$. In fact $P_{04}^{(z)}$ is closely related to the Green's function at the point $z$. Using information derived from these functions we define local derivatives $\partial_{2} f(z)$ and $\partial_{3} f(z)$ analogous to the normal and tangential derivatives (1.9) and (1.10) at a boundary point, and show that these derivatives exist if $f \in \operatorname{dom} \Delta$. We also identify $\Delta P_{0 k}^{(z)}$ for $k=4,5,6$ as "distributions supported at the point $z$," with $\Delta P_{04}^{(z)}$ a multiple of $\delta_{z}, \Delta P_{05}^{(z)}$ a multiple of $\partial_{2} \delta_{z}$, and $\Delta P_{06}^{(z)}$ a multiple of $\partial_{3} \delta_{z}$.

It is natural to try to extend the family $\left\{P_{0 k}^{(z)}\right\}$ of harmonic functions to a family $\left\{P_{j k}^{(z)}\right\}$ of monomials with

$$
\Delta P_{j k}^{(z)}=P_{(j-1) k}^{(z)} \quad \text { on } K \backslash\{z\}
$$

and

$$
P_{j k}^{(z)} \circ\left(F_{w}\right)^{n}=\left(5^{-j m} \sigma_{k}\right)^{n} P_{j k}^{(z)} .
$$

To keep the complexity manageable we restrict our attention to the case $w=(0,1)$ in section 5. The idea is to construct $P_{j k}^{(z)}$ first on the complement of the level $m$ cell $C$ containing $z$. We split $K \backslash C$ into a finite union of cells and use the bases of monomials from part I on each cell. We then use (1.18) to extend $P_{j k}^{(z)}$ to $K \backslash\{z\}$. In the process we require certain matching conditions, and this leads to a complicated set of linear equations with the number of equations equal to the number of free parameters in the construction. It is not clear how to obtain a proof of existence and uniqueness for these equations, but in the case $w=(0,1)$ we were able to write a program to solve these equations numerically.

Another important class of elementary functions are the eigenfunctions of the Laplacian,

$$
-\Delta u=\lambda u \text {. }
$$

Global solutions to (1.19) are described by the method of spectral decimation FS. (See also [T1 and [DSV].) To each $\lambda$ there exists a sequence $\left\{\lambda_{m}\right\}$ for $m \geq m_{0}$ (called the "generation of birth") such that

$$
\lambda=\lim _{m \rightarrow \infty} \frac{3}{2} 5^{m} \lambda_{m}
$$

and

$$
\lambda_{m}=\lambda_{m+1}\left(5-\lambda_{m+1}\right) \text { for } m \geq m_{0} .
$$

Then $\left.u\right|_{V_{m}}$ is a discrete eigenfunction on $\Gamma_{m}$ with eigenvalue $\lambda_{m}$ for $m \geq m_{0}$. Moreover $\lambda_{m}$ does not equal any of the exceptional values 2, 5,6 for $m>m_{0}$, while $\lambda_{m_{0}}$ is one of the exceptional values unless $m_{0}=1$. The case $m_{0}=1$ is called generic. Note that we can solve (1.21),

$$
\lambda_{m+1}=\frac{5+\varepsilon_{m} \sqrt{25-4 \lambda_{m}}}{2}
$$

for $\varepsilon_{m}= \pm 1$, and we must have $\varepsilon_{m}=-1$ for all but a finite number of $m$ 's in order for the limit in (1.20) to exist. We can also solve the discrete eigenvalue equation 
to extend the values of $u$ from $V_{m-1}$ to $V_{m}$. This is a local extension algorithm, so that if $|w|=m-1$ we have

$u\left(F_{w} F_{0} q_{1}\right)=\frac{\left(4-\lambda_{m}\right)}{\left(2-\lambda_{m}\right)\left(5-\lambda_{m}\right)}\left[u\left(F_{w} q_{0}\right)+u\left(F_{w} q_{1}\right)\right]+\frac{2}{\left(2-\lambda_{m}\right)\left(5-\lambda_{m}\right)} u\left(F_{w} q_{2}\right)$

and similar identities at $F_{w} F_{1} q_{2}$ and $F_{w} F_{2} q_{0}$, so the values of $u$ on the boundary of a cell of level $m$ are obtained from the values of $u$ on the boundary of the cell of level $m-1$ containing it by multiplying by a certain $3 \times 3$ matrix. (The matrix depends on $\lambda_{m}$ and the position of the cells.) By inverting the matrix we can similarly expand outward from the small cell to the large cell. Note that $\lambda_{m} \neq 2,5$ is required for (1.23) to be meaningful, and $\lambda_{m} \neq 6$ is required for the matrix to be invertible.

In section 6 , we show how to modify the method of spectral decimation to describe all solutions of the eigenvalue equation (1.19) allowing a singularity at a boundary point, for generic eigenvalues. Allowing the singularity increases the dimension of solutions from 3 to 4 , so we just need to describe one additional eigenfunction. We show that it is skew-symmetric with respect to $R_{0}$ and has a pole with the same rate of growth as $P_{04}^{(0)}$. In principle there should be a 6 -dimensional space of solutions to (1.19) with a singularity at a nonjunction point. For the periodic point $z$ discussed in section 5 , we can use power series

$$
\sum_{j=0}^{\infty}(-\lambda)^{j} P_{j k}^{(z)} \quad \text { for } k=1,2, \ldots, 6
$$

to give a basis, provided $|\lambda|$ is small enough and the growth rates suggested in section 5 are valid. Nevertheless, it would still be desirable to give a spectral decimation description in this case.

In part I there is a description of the solutions to the eigenvalue equation (1.19) on the infinite blow-up $\bigcup_{n=0}^{\infty} F_{0}^{-n} K$ of SG. However, this is only one of uncountably many distinct infinite blow-ups of SG. For any infinite word $w$, one can form the blow-up

$$
\mathrm{SG}_{w}=\bigcup_{n=0}^{\infty} F_{w_{1}}^{-1} F_{w_{2}}^{-1} \cdots F_{w_{n}}^{-1} K \quad \text { (increasing union), }
$$

and words that differ for all but a finite number of places yield distinct blow-ups. The one studied in part I is characterized as having a boundary point. If the word $w$ omits one digit, say 2, then the blow-up has a bottom line. It is easy to extend the method of spectral decimation to all infinite blow-ups, allowing the index $m$ to range over all integers, and allowing $m_{0}=-\infty$. (These will be the generic ones.) As in part I, we will be interested in picking out specific eigenfunctions with different boundedness and asymptotic behaviors "at infinity."

In section 7 , we study the case of generic eigenvalues. We show that in the case that the blow-up has a bottom line, there exists an eigenfunction that is constant along this line. This is closely related to the observation in [DSV] that certain Dirichlet eigenfunctions are constant along the "inner triangle" in SG, and may be explained by symmetry considerations as mentioned in [ASST]. For a blow-up in which all digits occur in any large enough segment, we are able to construct a basis of eigenfunctions where each eigenfunction goes to infinity in one "direction," and goes to zero in the other two directions. For this we need to assume not only that 
the eigenvalue is generic, but also that it is non-Julia, meaning that the discrete eigenvalues $\lambda_{j}$ do not belong to the Julia set $\mathcal{J}$ of the polynomial $z(5-z)$. This consideration is equivalent to saying $\left|\lambda_{j}\right|$ goes to infinity as $j \rightarrow-\infty$.

In section 8 , we study some of the eigenvalues omitted in section 7 . For nongeneric $\lambda$, many cases are described in [T1] since they contribute to the $L^{2}$ spectrum of the blow-up. We are able to give a complete description, including the case when some $\lambda_{m}=2$ not considered in T1. When $\lambda_{m} \in \mathcal{J}$ the situation is more subtle. The fixed point $4 \in \mathcal{J}$ seems to be special. If $\lambda_{m}=4$, then all eigenfunctions are bounded. We conjecture that if $\lambda_{m} \in \mathcal{J}$ but $\lambda_{m} \neq 4$ (for all $m \leq m_{0}$ ), then there are no bounded eigenfunctions (for a blow-up with no boundary point). We are able to prove that there are no bounded eigenfunctions if $\lambda_{m}=3 \pm \sqrt{3}$. (This is the two-cycle in $\mathcal{J}$.)

To put these matters in context, we should consider what happens in the theory of Riemannian manifolds without boundary. The $L^{2}$ spectral resolution of the Laplacian typically gives rise to many bounded eigenfunctions, and we can obtain more by taking a suitable closure. Are there any more bounded eigenfunctions? The answer depends a lot on the particular manifold. (In general, the answer is unknown.) For Euclidean space, the answer is no. For hyperbolic space, the answer is yes, and in fact there is a whole analytic family of them. So the case of $\mathbf{S G}_{w}$ seems to fall in between these extremes.

In section 9, we study normal derivatives at the boundary for Dirichlet eigenfunctions and heat kernels. For comparison, note that on the unit interval the functions $\left\{\frac{1}{\sqrt{2}} \sin \pi j x\right\}$ form an orthonormal basis of Dirichlet eigenfunctions with eigenvalue $(\pi j)^{2}$ and normal derivatives $-\pi j / \sqrt{2}$ and $(-1)^{j+1} \pi j / \sqrt{2}$ at 0 and 1 , so that $\left|\partial_{n} u\right|=c \sqrt{\lambda}$ exactly. On SG we are able to compute the normal derivatives of all normalized Dirichlet eigenfunctions using spectral decimation and some results about inner products from OSS. The answer is given in terms of infinite products involving the sequence $\left\{\lambda_{m}\right\}$ given by (1.20) and (1.21). Using this we are able to prove the upper bound

$$
\left|\partial_{n} u\right| \leq c \sqrt{\lambda},
$$

but this is far from the entire story. For most eigenvalues, the normal derivatives are actually much smaller than the bound in (1.24). By looking at graphs of the ratio $\partial_{n} u / \sqrt{\lambda}$ versus $\lambda$ we see that there is a very rich self-similar structure to the data. Also, there is a large space of joint Dirichlet-Neumann eigenfunctions for which the normal derivatives are zero.

We are also interested in normal derivatives of the Dirichlet heat kernel

$$
p_{t}(x, y)=\sum e^{-\lambda_{j} t} u_{j}(x) u_{j}(y),
$$

where $\left\{u_{j}\right\}$ denotes an orthonormal basis of Dirichlet eigenfunctions with eigenvalues $\left\{\lambda_{j}\right\}$. In particular, we want to take normal derivatives with respect to both variables at the same boundary point (say $q_{0}$ ), so

$$
\partial_{n} \partial_{n} p_{t}\left(q_{0}, q_{0}\right)=\sum e^{-\lambda_{j} t}\left(\partial_{n} u_{j}\left(q_{0}\right)\right)^{2} .
$$

This quantity plays an important role in analysis on the product space $\mathrm{SG} \times \mathrm{SG}$ developed in [S6]. Sharp estimates for the heat kernel and its time derivatives are known (see [Ba, Ki2] and the references there), but nothing is known about (1.26). 
Certain estimates were conjectured in [S6], namely

$$
\partial_{n} \partial_{n} p_{t}\left(q_{0}, q_{0}\right) \leq c t^{-\log (25 / 3) / \log 5} \text { for } 0<t \leq 1 .
$$

Here we give strong experimental evidence that (1.27) holds. It is important to note that the estimate (1.24) is not adequate to prove (1.27). In fact, our evidence suggests that $t^{\log (25 / 3) / \log 5} \partial_{n} \partial_{n} p_{t}\left(q_{0}, q_{0}\right)$ behaves asymptotically like a periodic function of $\log 1 / t$ as $t \rightarrow 0$. This suggests that a similar statement will hold for the on-diagonal heat kernel $p_{t}(x, x)$ (with a different exponent of $t$ ). This is shown in $\mathrm{AS}$.

Taken together, the results of this paper and part I give us a reasonably detailed understanding of some elementary special functions on SG (see also A-LS). On the other hand, this is not enough. Because of the negative results in [BST], we cannot compose any of these functions with a nonlinear real-valued function and stay within $\operatorname{dom} \Delta$. For example, here is a challenging problem that apparently cannot be solved using just these elementary functions: find the analog on SG to the famous theorem of Borel that there exist $C^{\infty}$ functions on the line with any prescribed Taylor expansion. A solution to this problem might pave the way for a full-fledged theory of distributions on SG.

This paper reports the results of certain numerical computations. For the actual programs used to generate these computations and further numerical results, the reader should consult the following web sites:

(1) www.math.cornell.edu/Bengal/ for monomials with boundary point singularity, and normal derivatives of eigenfunctions and heat kernels;

(2) www.math.cornell.edu/abbysk/ for monomials with periodic point singularity;

(3) www.math.cornell.edu/bj94192/ for eigenfunctions on blowups.

\section{HARMONIC FUNCTIONS WITH POINT SINGULARITY}

A harmonic function on $K$ is defined to be a continuous function $h$ satfisfying the discrete harmonic condition

$$
\Delta_{m} h(x)=0 \quad \text { for all } x \in V_{k} \backslash V_{0} \quad \text { for all } k \text { and } m \text { with } m \geq k .
$$

The boundary points are treated exceptionally, since if we tried to impose (2.1) at boundary points (this is equivalent to imposing Neumann boundary conditions) the only harmonic functions would be the constants. On the other hand, the requirement that $h$ be continuous at boundary points is a condition of a different nature than the continuity on $K \backslash V_{0}$. Indeed, the discrete harmonic condition (2.1) already implies that $h$ is continuous on $V_{*} \backslash V_{0}$ and extends continuously to $K \backslash V_{0}$. (One should delete the cases of (2.1) that involve values on $V_{0}$.) But it has been known since [DSV] that there exist functions that are unbounded in a neighborhood of points in $V_{0}$ that are otherwise harmonic. These are the prototypes of the functions we study in this section.

We begin with a more general situation. Let $\Omega$ be any open subset of $K$, and let $S=K \backslash \Omega$ be its closed complement (the singularity set).

Definition 2.1. A function $h$ on $\Omega$ is said to be harmonic on $\Omega$ if $h$ is continuous on $\Omega$ and $h$ satisfies (2.1) at every point $x \in V_{k} \cap \Omega \backslash V_{0}$ for all $m$ large enough that $U_{m}(x) \subseteq \Omega$. Recall that $U_{m}(x)$ is the standard neighborhood consisting of the two 
cells of level $m$ having $x$ as boundary point. Since $\Omega$ is open, we have $U_{m}(x) \subseteq \Omega$ for all large enough $m$.

The results of [DSV] as elaborated in [KSS] says that if $\Omega=K \backslash\left\{q_{0}\right\}$, then the space of harmonic functions on $\Omega$ is 4-dimensional, with a basis $h_{1}, h_{2}, h_{3}, h_{4}$, and $h_{1}, h_{2}, h_{3}$ span the 3 -dimensional space of harmonic functions of $K$. The functions $h_{1}$ and $h_{2}$ are even with respect to $R_{0}$, with $h_{1} \equiv 1$ and $h_{2}$ having boundary values $h_{2}\left(q_{0}\right)=0, h_{2}\left(q_{1}\right)=h_{2}\left(q_{2}\right)=1$. The functions $h_{3}$ and $h_{4}$ are odd with respect to $R_{0}$, with $h_{3}$ having boundary values $h_{3}\left(q_{0}\right)=0, h_{3}\left(q_{1}\right)=-h_{3}\left(q_{2}\right)=1$, while $h_{4}$ satisfies

$$
h_{4}\left(F_{0}^{m} q_{1}\right)=-h_{4}\left(F_{0}^{m} q_{2}\right)=3^{m} .
$$

Note that $h_{4}$ just barely fails to be integrable on $K$. The functions $h_{j}$ are all harmonic on $F_{1} K \cup F_{2} K$ and are uniquely determined by their boundary values at the four boundary points $\left(q_{1}, q_{2}, F_{0} q_{1}, F_{0} q_{2}\right)$. The most important property of these functions is the scaling identity

$$
h_{j}\left(F_{0}^{m} x\right)=\sigma_{j}^{m} h_{j}(x) \text { for } \sigma_{1}=1, \sigma_{2}=\frac{3}{5}, \sigma_{3}=\frac{1}{5}, \sigma_{4}=3 .
$$

It is useful to be able to localize the definition. Suppose $\Omega$ is an open subset of any cell $F_{w} K$. Then a continuous function $h$ on $\Omega$ is harmonic on $\Omega$ in $F_{w} K$ if (2.1) holds for $x \in V_{k} \cap \Omega \backslash F_{w} V_{0}$ provided $U_{m}(x) \subseteq \Omega$. It is easy to see that this is equivalent to saying that $h \circ F_{w}^{-1}$ is harmonic on $F_{w}^{-1} \Omega$ in $K$.

Our first result is a simple extension theorem.

Theorem 2.2. Suppose $S$ is closed in $F_{w} K$ and $S$ is disjoint from the boundary $F_{w} V_{0}$. Then any harmonic function on $F_{w} K \backslash S$ in $F_{w} K$ extends uniquely to a harmonic function on $K \backslash S$.

Proof. It clearly suffices to prove this for $F_{0} K$. So let $h$ be harmonic on $F_{0} K \backslash$ $S$. By hypothesis $F_{0} q_{1}$ and $F_{0} q_{2}$ are disjoint from $S$, so the values $h\left(F_{0} q_{1}\right)=$ $c$ and $h\left(F_{0} q_{2}\right)=d$ are specified. If we choose values $h\left(q_{1}\right)=a$ and $h\left(q_{2}\right)=$ $b$, then this will determine a unique harmonic function on $F_{1} K \cup F_{2} K$, and the extension will be harmonic on $K \backslash S$ if and only if the normal derivatives satisfy the matching conditions at $F_{0} q_{1}$ and $F_{0} q_{2}$. (See Figure 2.1.) This gives us two linear equations in $a$ and $b$, so we can reasonably expect a unique solution. Note that $h\left(F_{1} q_{2}\right)=\frac{1}{4}(a+b+c+d)$, so the normal derivative at $F_{0} q_{1}$ with respect to the $F_{1} K$ cell is $\frac{5}{3}\left(2 c-a-\frac{a+b+c+d}{4}\right)$, and similarly the normal derivative at $F_{0} q_{2}$ with respect to the $F_{2} K$ cell is $\frac{5}{3}\left(2 d-b-\frac{a+b+c+d}{4}\right)$, and by inspection these are linearly independent in the $(a, b)$ variables.

It is not difficult to see that even if $S$ intersects $F_{w} V_{0}$, the extension is still possible, but the uniqueness fails.

We may use this extension theorem to localize the description of harmonic functions with singularities. The following is a typical application.

Corollary 2.3. Let $\left\{x_{1}, x_{2}, \ldots, x_{N}\right\}$ be any finite set of distinct nonjunction points in $K$. Then any harmonic function in $K \backslash\left\{x_{1}, x_{2}, \ldots, x_{N}\right\}$ may be written $\sum_{n=1}^{N} u_{n}$ for $u_{n}$ harmonic in $K \backslash\left\{x_{n}\right\}$, and moreover $u_{n}$ is unique modulo global harmonic functions.

Proof. Since the points $x_{j}$ are not junction points, we can find cells $F_{w(j)} K$ containing $x_{j}$ but no other $x_{k}(k \neq j)$. Let $h$ be the harmonic function on 


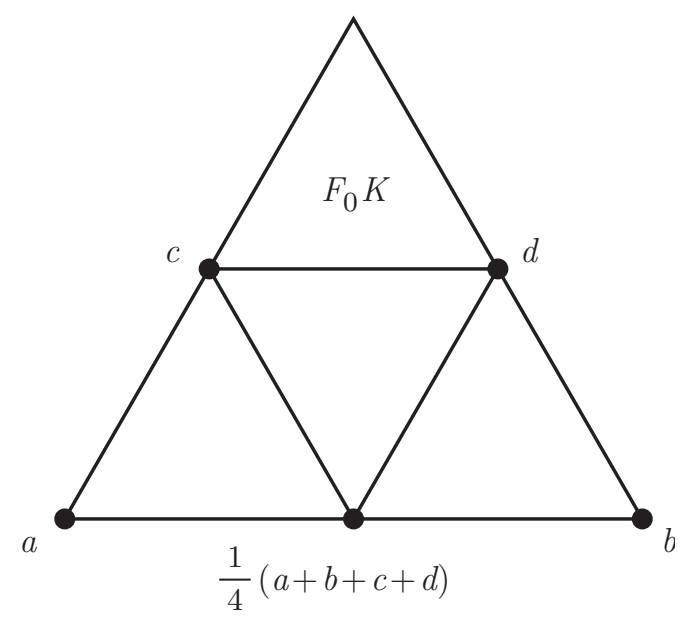

Figure 2.1.

$K \backslash\left\{x_{1}, x_{2}, \ldots, x_{n}\right\}$. Then $h$ restricted to $F_{w(1)} K$ is harmonic on $F_{w(1)} K \backslash\left\{x_{1}\right\}$. We take $u_{1}$ to be the extension to a harmonic function on $K \backslash\left\{x_{1}\right\}$. Then $h-u_{1}$ vanishes on $F_{w(1)} K$, so it is harmonic on $K \backslash\left\{x_{2}, x_{3}, \ldots, x_{N}\right\}$. Then for $u_{2}$ we take the harmonic extension of $h-u_{1}$ from $F_{w(2)} K \backslash\left\{x_{2}\right\}$ to $K \backslash\left\{x_{2}\right\}$. Thus $h-u_{1}-u_{2}$ is harmonic on $K \backslash\left\{x_{3}, x_{4}, \ldots, x_{N}\right\}$. Continuing in this way we obtain existence. To show uniqueness assume $\sum u_{n}$ and $\sum u_{n}^{\prime}$ are two such representations, with $u_{n}$ and $u_{n}^{\prime}$ both harmonic in $K \backslash\left\{x_{n}\right\}$. Then $u_{k}-u_{k^{\prime}}=\sum_{n \neq k}\left(u_{n}^{\prime}-u_{n}\right)$ is harmonic in both $K \backslash\left\{x_{k}\right\}$ and $K \backslash\left\{x_{1}, x_{2}, \ldots, \hat{x}_{k}, \ldots, x_{N}\right\}$, hence is a global harmonic function.

This localization is also valid if we allow junction point singularities, as we will see later.

Theorem 2.4. Let $x_{0}$ be any nonboundary junction point. Then the space of harmonic functions on $K \backslash\left\{x_{0}\right\}$ is 7-dimensional.

Proof. By Theorem 2.2 it suffices to prove this for a level 1 junction point, say $x_{0}=F_{1} q_{2}=F_{2} q_{1}$. Let $H$ denote the space of harmonic functions on $K \backslash\left\{F_{1} q_{2}\right\}$. We also consider the space $F_{0} K \cup F_{1} F_{0} K \cup F_{1} F_{1} K \cup F_{2} F_{0} K \cup F_{2} F_{2} K$ (that is $K$ with the interior of $F_{1} F_{2} K \cup F_{2} F_{1} K$ deleted) as a fractafold with boundary $\left\{q_{0}, q_{1}, q_{2}, F_{1} F_{2} q_{0}, F_{1} F_{2} q_{1}, F_{2} F_{1} q_{0}, F_{2} F_{1} q_{2}\right\}$. See Figure 2.2. Let $H_{1}$ denote the 7-dimensional space of harmonic functions on this fractafold. Clearly, restriction maps $H$ to $H_{1}$. To complete the proof we need to show that this is invertible, and we do this by exhibiting an explicit, unique extension from $H_{1}$ to $H$. Let $h \in H_{1}$. Because the functions in $H$ are unrestricted at $F_{1} q_{2}$, we simply have to extend the function $h$ restricted to $F_{1} F_{0} K \cup F_{1} F_{1} K$ to a function harmonic on $F_{1} K \backslash\left\{F_{1} q_{2}\right\}$, and also the function $h$ restricted to $F_{2} F_{0} K \cup F_{2} F_{2} K$ to a function harmonic on $F_{2} K \backslash\left\{F_{2} q_{1}\right\}$, with no compatibility requirement on the two extensions. Of course, both extension problems are localized versions of the extension from $F_{1} K \cup F_{2} K$ to $K \backslash\left\{q_{0}\right\}$, and this has a unique solution that can be given explicitly by the basis $h_{1}, h_{2}, h_{3}, h_{4}$ described above. 


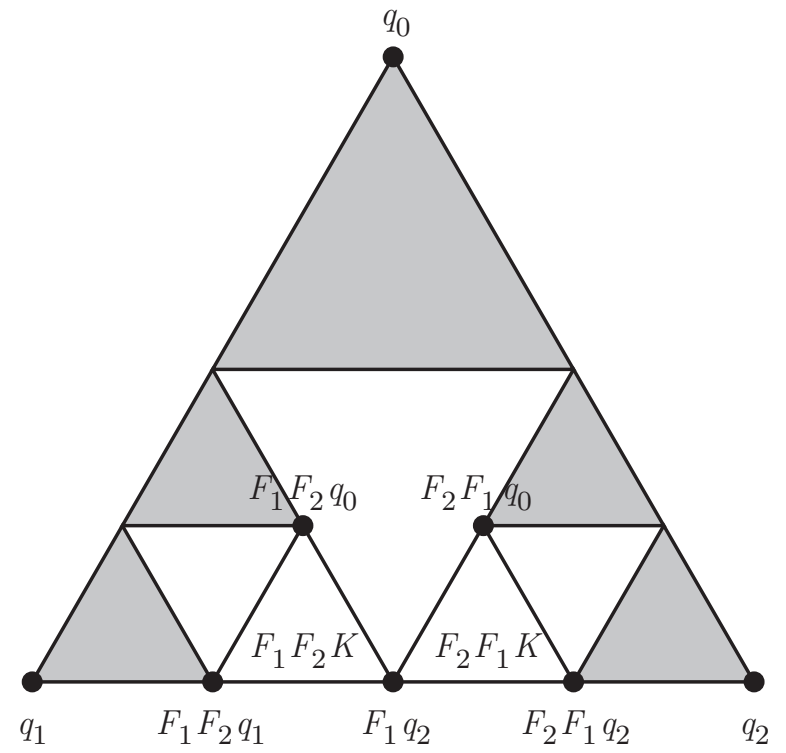

FIGURE 2.2.
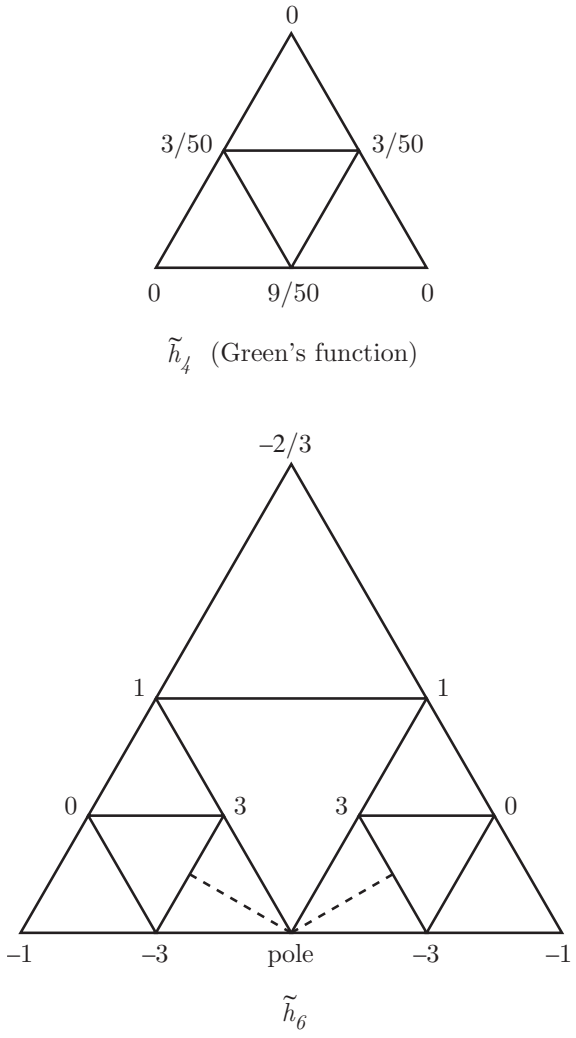
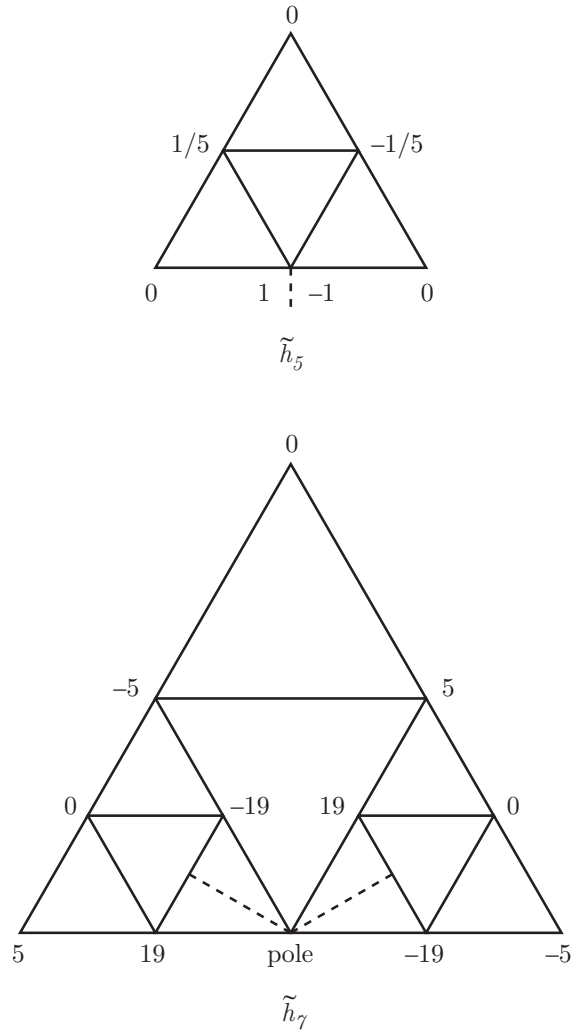

Figure 2.3. 
The 7-dimensional space for $K \backslash\left\{F_{1} q_{2}\right\}$ splits into a 4-dimensional space symmetric with respect to $R_{0}$ and a 3 -dimensional skew-symmetric space. In addition to $h_{1}, h_{2}$ (symmetric) and $h_{3}$ (skew-symmetric) which generate global harmonic functions, we can complete a basis with $\widetilde{h}_{4}$ (continuous symmetric), $\widetilde{h}_{5}$ (bounded, discontinuous at $F_{1} q_{2}$, skew-symmetric), $\widetilde{h}_{6}$ (unbounded, symmetric) and $\widetilde{h}_{7}$ (unbounded, skew-symmetric) as shown in Figure 2.3.

Another way to describe $H$ is in terms of restrictions to $F_{1} K \backslash\left\{F_{1} q_{2}\right\}$ and $F_{2} K \backslash\left\{F_{2} q_{1}\right\}$. Each of these restriction spaces is 4-dimensional, which means there must be a 1-dimensional space of obstructions to extending to $F_{0} K$. But in fact we know that $h_{4}$ does not extend to a neighborhood of $q_{0}$, and in fact the 8-dimensional space generated by $h_{1}, h_{2}, h_{3}, h_{4}, \widetilde{h}_{4}, \widetilde{h}_{5}, \widetilde{h}_{6}, \widetilde{h}_{7}$ gives the space of harmonic functions on $K \backslash\left\{q_{0}, F_{1} q_{2}\right\}$.

Theorem 2.5. Let $x_{0}$ be any nonjunction point. Then the space of harmonic functions on $K \backslash\left\{x_{0}\right\}$ is 6 -dimensional, with each such function determined uniquely by the values of $h\left(q_{j}\right)$ and $\partial_{n} h\left(q_{j}\right), j=0,1,2$.

Proof. Let $\left(w_{1}, w_{2}, \ldots\right)$ denote the infinite word uniquely representing $x_{0}$, so $x_{0} \in$ $F_{w_{1}} F_{w_{2}} \cdots F_{w_{m}} K$ for all $m$. Let $\mathcal{F}_{m}$ denote the fractafold obtained by deleting the interior of $F_{w_{1}} F_{w_{2}} \cdots F_{w_{m}} K$ from $K$, where $m$ is large enough that the sequence $\left(w_{1}, w_{2}, \ldots, w_{m}\right)$ is not constant. Then $\mathcal{F}_{m}$ has 6 boundary points, $q_{j}$ and $F_{w_{1}} F_{w_{2}} \cdots F_{w_{m}} q_{j}$ for $j=0,1,2$. Let $H_{m}$ denote the 6-dimensional space of harmonic functions on $\mathcal{F}_{m}$. We claim that the data $\left\{h\left(q_{j}\right), \partial_{n} h\left(q_{j}\right)\right\}=\left\{a_{j}, n_{j}\right\}$ for $j=0,1,2$ uniquely determine $h \in H_{m}$.

First we show that $\left\{a_{j}, n_{j}\right\}$ uniquely determines

$$
\left\{h\left(F_{w_{1}} q_{j}\right), \partial_{n} h\left(F_{w_{1}} q_{j}\right)\right\}=\left\{a_{j}^{\prime}, n_{j}^{\prime}\right\} .
$$

For simplicity assume $w_{1}=0$. Then $\left\{a_{0}, n_{0}\right\}=\left\{a_{0}^{\prime}, n_{0}^{\prime}\right\}$. For $h$ to be harmonic at $F_{1} q_{2}=F_{2} q_{1}$ we must have

$$
h\left(F_{1} q_{2}\right)=\frac{1}{4}\left(a_{1}+a_{2}+a_{1}^{\prime}+a_{2}^{\prime}\right)
$$

(see Figure 2.4). By the definition of normal derivative we have

$$
\left\{\begin{array}{l}
\frac{3}{5} n_{1}=2 a_{1}-a_{1}^{\prime}-\frac{1}{4}\left(a_{1}+a_{2}+a_{1}^{\prime}+a_{2}^{\prime}\right) \\
\frac{3}{5} n_{2}=2 a_{2}-a_{2}^{\prime}-\frac{1}{4}\left(a_{1}+a_{2}+a_{1}^{\prime}+a_{2}^{\prime}\right)
\end{array}\right.
$$

and the matching conditions for normal derivatives at $F_{0} q_{1}$ and $F_{0} q_{2}$ yield

$$
\left\{\begin{array}{l}
-\frac{3}{5} n_{1}^{\prime}=2 a_{1}^{\prime}-a_{1}-\frac{1}{4}\left(a_{1}+a_{2}+a_{1}^{\prime}+a_{2}^{\prime}\right), \\
-\frac{3}{5} n_{2}^{\prime}=2 a_{2}^{\prime}-a_{2}-\frac{1}{4}\left(a_{1}+a_{2}+a_{1}^{\prime}+a_{2}^{\prime}\right) .
\end{array}\right.
$$

More precisely, any $h \in H_{m}$ satisfies (2.4) and (2.5), and conversely, if (2.4) and (2.5) hold, there exists a harmonic function on $F_{1} K \cup F_{2} K$ satisfying $\left\{h\left(q_{j}\right), \partial_{n} h\left(q_{j}\right)\right\}$ $=\left\{a_{j}, n_{j}\right\}$ for $j=1,2$ and $\left\{h\left(F_{j} q_{0}\right), \partial_{n} h\left(F_{j} q_{0}\right)\right\}=\left\{a_{j}^{\prime}, n_{j}^{\prime}\right\}$. But it is easy to see that the equations (2.4) and (2.5) allow a unique solution for primed variables in terms of unprimed variables, and vice versa.

By iterating the above argument we see that for $f \in H_{m}$ the initial data uniquely determines the data $\left\{h\left(F_{w_{1}} F_{w_{2}} \cdots F_{w_{m}} q_{j}\right), \partial_{n} h\left(F_{w_{1}} F_{w_{2}} \cdots F_{w_{m}} q_{j}\right)\right\}$, and vice versa, and in fact there exists $f \in H_{m}$ with any initial data. Now if $h \in H$, then its restriction to $\mathcal{F}_{m}$ is in $H_{m}$, and conversely, if the restriction of $h$ to $\mathcal{F}_{m}$ is in $H_{m}$ 


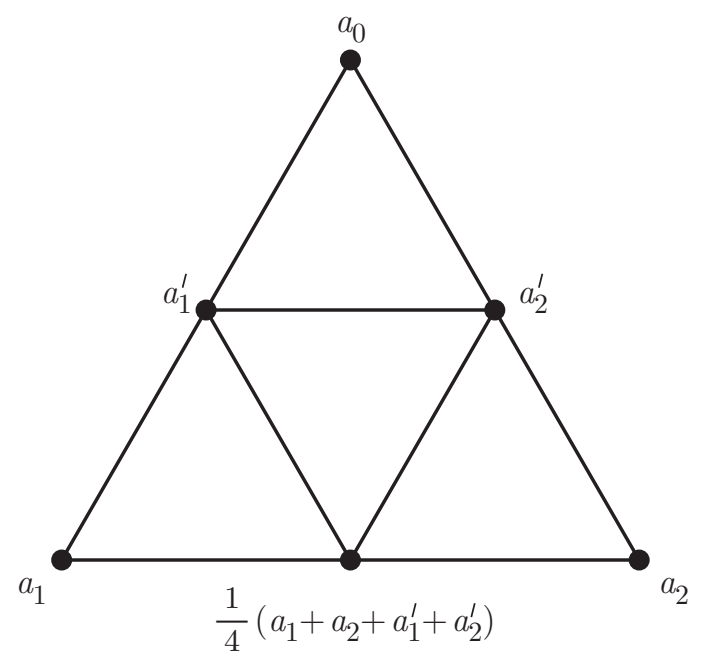

FIGURE 2.4.

for all $m$, then $h \in H$. This allows us to construct a function in $H$ from any initial data by successively defining it on $\mathcal{F}_{m}$, so $H$ is at least 6 -dimensional. But if the initial data is zero, then $h$ must vanish on all $\mathcal{F}_{m}$, hence $h$ is zero, so $H$ is exactly 6-dimensional.

Theorem 2.6. Given distinct points $\left\{x_{1}, x_{2}, \ldots, x_{N}\right\}$, let $N=N_{1}+N_{2}+N_{3}$, where $N_{1}$ is the number of boundary points $\left(N_{1} \leq 3\right), N_{2}$ is the number of nonboundary junction points, and $N_{3}$ is the number of nonjunction points. The space of harmonic functions on $K \backslash\left\{x_{1}, x_{2}, \ldots, x_{N}\right\}$ has dimension $3+N_{1}+4 N_{2}+3 N_{3}$.

Proof. For each sufficiently large $m$, let $\mathcal{F}_{m}$ denote the fractafold obtained from $K$ by removing a level $m$ neighborhood of each $x_{j}$ as follows: (i) if $x_{j}$ is a boundary point (say $x_{j}=q_{j}$ ), remove the interior of $F_{j}^{m} K$ and also the point $q_{j}$; (ii) if $x_{j}$ is a nonboundary junction point, remove $U_{m}\left(x_{j}\right)$; (iii) if $x_{j}$ is a nonjunction point determined by the infinite word $\left(w_{1}, w_{2}, \ldots\right)$, remove the interior of $F_{w_{1}} F_{w_{2}} \cdots F_{w_{m}} K$.

Let $H_{m}$ denote the space of harmonic functions on $\mathcal{F}_{m}$. Note that once $m$ is large enough that the deleted neighborhoods have disjoint closures (not containing any boundary points in cases (ii) and (iii)), the boundary of $\mathcal{F}_{m}$ consists of exactly $3+N_{1}+4 N_{2}+3 N_{3}$ points, so this is the dimension of $H_{m}$. Reasoning as before, each function in $H_{m}$ has a unique extension to a function in $H_{m+1}$, and any function in $H$ restricted to $\mathcal{F}_{m}$ yields a function in $H_{m}$. The rest of the argument is the same as before.

\section{HARMONIC FUnCTIONS WITH SINGULARITY AT A PERIODIC POINT}

Let $w$ denote a word of finite length $m$. A periodic point $z$ is a point with address $(w, w, w, \ldots)$, or equivalently, the fixed-point of $F_{w}$. We would like to describe in detail the 6-dimensional space of functions harmonic on $K \backslash\{z\}$. We want to find a basis $\left\{P_{0 k}^{(z)}\right\}, k=1,2, \ldots, 6$, characterized by the scaling identity

$$
P_{0 k}^{(z)} \circ\left(F_{w}\right)^{n}=\sigma_{k}^{n} P_{0 k}^{(z)}
$$


for specific factors $\sigma_{k}$. For $k=1,2,3$, these functions will be globally harmonic, while for $k=4,5,6$ they will have nonremovable singularities. According to Theorem 2.5 , the values $\left\{h\left(q_{j}\right)\right\}$ and $\left\{\partial_{n} h\left(q_{j}\right)\right\}$ uniquely determine a harmonic function on $K \backslash\{z\}$. We need to understand the linear transformation that describes the mapping $h \rightarrow h \circ F_{w}$ in these coordinates.

We begin by considering just the 3 -dimensional space $\mathcal{H}_{0}$ of global harmonic functions. Then we know

$$
n=D a, \quad \text { where } n=\left(\begin{array}{l}
\partial_{n} h\left(q_{0}\right) \\
\partial_{n} h\left(q_{1}\right) \\
\partial_{n} h\left(q_{2}\right)
\end{array}\right), a=\left(\begin{array}{l}
h\left(q_{0}\right) \\
h\left(q_{1}\right) \\
h\left(q_{2}\right)
\end{array}\right) \text { and } D=\left(\begin{array}{ccc}
2 & -1 & -1 \\
-1 & 2 & -1 \\
-1 & -1 & 2
\end{array}\right) \text {. }
$$

Let $a_{j}^{\prime}=h\left(F_{w_{1}} q_{j}\right)$ and $n_{j}^{\prime}=\partial_{n} h\left(F_{w_{1}} q_{j}\right)=\frac{5}{3} \partial_{n}\left(h \circ F_{w_{1}}\right)\left(q_{j}\right)$. Then

$$
\left\{\begin{array}{l}
a^{\prime}=A_{w_{1}} a \\
n^{\prime}=\frac{5}{3} D A_{w_{1}} a
\end{array}\right.
$$

where $A_{i}$ are the harmonic extension matrices,

$$
A_{0}=\frac{1}{5}\left(\begin{array}{lll}
5 & 0 & 0 \\
2 & 2 & 1 \\
2 & 1 & 2
\end{array}\right)
$$

etc. More generally, by iteration

$$
\left\{\begin{array}{l}
\widetilde{a}=\left(A_{w_{m}} A_{w_{m-1}} \cdots A_{w_{1}}\right) a \\
\widetilde{n}=\left(\frac{5}{3}\right)^{m} D\left(A_{w_{m}} A_{w_{m-1}} \cdots A_{w_{1}}\right) a
\end{array}\right.
$$

where $\widetilde{a}_{j}=h\left(F_{w} q_{j}\right)$ and $\tilde{n}=\partial_{n} h\left(F_{w} q_{j}\right)$.

If $a^{(k)}$ is an eigenvector for the matrix $A_{w_{m}} A_{w_{m-1}} \cdots A_{w_{1}}$ with eigenvalue $\sigma_{k}$, then $h$ will satisfy (3.1). Let $\sigma_{1}=1, \sigma_{2}, \sigma_{3}$ denote the eigenvalues of this matrix. $\left(\sigma_{1}\right.$ corresponds to the eigenvector $\left(\begin{array}{l}1 \\ 1 \\ 1\end{array}\right)$.) Since $\operatorname{det} A_{w_{m}} A_{w_{m-1}} \cdots A_{w_{1}}=\left(\frac{3}{25}\right)^{m}$, we have $\sigma_{2} \sigma_{3}=\left(\frac{3}{25}\right)^{m}$, and also

$$
\left(\frac{1}{5}\right)^{m}<\left|\sigma_{k}\right|<\left(\frac{3}{5}\right)^{m} \quad \text { for } k=2,3 .
$$

The strict inequalities in (3.5) of course require that not all $w_{j}$ are equal. Typically, these will be two distinct real eigenvalues, so we may order them, $\sigma_{1}>\sigma_{2}>\sigma_{3}$. There is at least one case $w=(0,1,2)$, when $\sigma_{2}$ and $\sigma_{3}$ are complex conjugate pairs, with $\left|\sigma_{2}\right|=\left(\frac{\sqrt{3}}{5}\right)^{m}$. (See A-LS for a detailed discussion of the case when $\sigma_{2}$ and $\sigma_{3}$ are complex conjugate pairs.) It does not appear that a multiplicity 2 eigenspace (or even generalized eigenspace) ever occurs. In the typical case, we will define $P_{0 k}^{(z)}$ for $k=1,2,3$ by taking initial values $a=a^{(k)}$. Note that $P_{01}^{(z)} \equiv 1$, while $P_{02}^{(z)}(z)=P_{03}^{(z)}(z)=0$, but the rate of vanishing is faster for $P_{03}^{(z)}$.

Next we examine what happens when we allow a singularity at $z$. This means (3.2) is no longer valid. It is convenient to define a perturbation vector $N$ to measure the deviation of $n$ from $D a$ :

$$
n=D a+N
$$

Similarly, $n^{\prime}=\frac{5}{3} D a^{\prime}+N^{\prime}$ and $\widetilde{n}=\left(\frac{5}{3}\right)^{m} D \widetilde{a}+\widetilde{N}$. We need to find the analog of (3.3). For simplicity set $w_{1}=0$. That means $z \in F_{0} K$, so $h$ is harmonic in 
$F_{1} K \cup F_{2} K$, so $h\left(F_{1} q_{2}\right)=\frac{1}{4}\left(a_{1}+a_{2}+a_{1}^{\prime}+a_{2}^{\prime}\right)$. Computing normal derivatives at $q_{1}$ and $q_{2}$ using the cells $F_{1} K$ and $F_{2} K$ yields

$$
\begin{aligned}
& n_{1}=\frac{5}{3}\left[2 a_{1}-a_{1}^{\prime}-\frac{1}{4}\left(a_{1}+a_{2}+a_{1}^{\prime}+a_{2}^{\prime}\right)\right], \\
& n_{2}=\frac{5}{3}\left[2 a_{2}-a_{2}^{\prime}-\frac{1}{4}\left(a_{1}+a_{2}+a_{2}^{\prime}+a_{2}^{\prime}\right)\right] .
\end{aligned}
$$

This allows us to solve for $a_{1}^{\prime}$ and $a_{2}^{\prime}$ in terms of $a_{1}, a_{2}, N_{1}, N_{2}$ (using (3.6)). Of course, $a_{0}^{\prime}=a_{0}$. We may write the solution in matrix notation

$$
a^{\prime}=A_{0} a+\frac{3}{5} B_{0} N
$$

for

$$
B_{0}=\left(\begin{array}{ccc}
0 & 0 & 0 \\
0 & -\frac{5}{6} & \frac{1}{6} \\
0 & \frac{1}{6} & -\frac{5}{6}
\end{array}\right) .
$$

We may also compute the normal derivatives $n_{1}^{\prime}$ and $n_{2}^{\prime}$ using the cells $F_{1} K$ and $F_{2} K$ (and taking the negative):

$$
\begin{aligned}
& n_{1}^{\prime}=-\frac{5}{3}\left[2 a_{1}^{\prime}-a_{1}-\frac{1}{4}\left(a_{1}+a_{2}+a_{1}^{\prime}+a_{2}^{\prime}\right)\right], \\
& n_{2}^{\prime}=-\frac{5}{3}\left[2 a_{2}^{\prime}-a_{2}-\frac{1}{4}\left(a_{1}+a_{2}+a_{2}^{\prime}+a_{2}^{\prime}\right)\right] .
\end{aligned}
$$

Using (3.7) to eliminate $a_{1}^{\prime}$ and $a_{2}^{\prime}$ and expressing the answer in terms of $N_{1}^{\prime}$ and $N_{2}^{\prime}$ we obtain

$$
\begin{aligned}
& N_{1}^{\prime}=\frac{10}{3} N_{1}-\frac{5}{3} N_{2}, \\
& N_{2}^{\prime}=-\frac{5}{3} N_{1}+\frac{10}{3} N_{2} .
\end{aligned}
$$

Also, since $n_{0}^{\prime}=n_{0}$ we have $N_{0}^{\prime}=N_{0}-\frac{2}{3} N_{1}-\frac{2}{3} N_{2}$. In matrix form this is simply

$$
N^{\prime}=\left(A_{0}^{\text {tr }}\right)^{-1} N \text {. }
$$

Iterating this argument, we obtain

$$
\begin{aligned}
& \widetilde{a}=\left(A_{w_{m}} A_{w_{m-1}} \cdots A_{w_{1}}\right) a+\widetilde{B} N, \\
& \widetilde{N}=\left(\left(A_{w_{m}} A_{w_{m-1}} \cdots A_{w_{1}}\right)^{\operatorname{tr}}\right)^{-1} N
\end{aligned}
$$

for a certain matrix $\widetilde{B}$. In order to have (3.1) hold we need $\widetilde{a}=\sigma_{k} a$ and $\widetilde{N}=$ $\left(\frac{5}{3}\right)^{m} \sigma_{k} N$. Of course, the eigenvalues of $\left(\left(A_{w_{m}} A_{w_{m-1}} \cdots A_{w_{1}}\right)^{\operatorname{tr}}\right)^{-1}$ are $1, \sigma_{1}^{-1}$, $\sigma_{2}^{-1}$. Write $N^{(4)}, N^{(5)}, N^{(6)}$ for corresponding eigenvectors. Then for $\sigma_{4}=\left(\frac{3}{5}\right)^{m}$, $\sigma_{5}=\left(\frac{3}{5}\right)^{m} \sigma_{2}^{-1}, \sigma_{6}=\left(\frac{3}{5}\right)^{m} \sigma_{3}^{-1}$, we take $N=N^{(k)}$ and determine $a^{(k)}$ by solving $\widetilde{a}=\sigma_{k} a$ and $(3.10)$ :

$$
\left(\sigma_{k} I-A_{w_{m}} A_{w_{m-1}} \cdots A_{w_{1}}\right) a=\widetilde{B} N
$$


We can check (using (3.5)) that $\sigma_{i} \sigma_{j} \neq\left(\frac{3}{5}\right)^{m}$ for $i, j \leq 3$, so (3.12) is solvable. We thus obtain $P_{0 k}^{(z)}$ for $k=4,5,6$ satisfying (3.1). Note that $\sigma_{4}<1$ while $\sigma_{5}>1$ and $\sigma_{6}>1$, so $P_{04}^{(z)}$ has a bounded singularity (in fact $\lim _{x \rightarrow z} P_{04}^{(z)}(x)=0$ ) while $P_{05}^{(z)}$ and $P_{06}^{(z)}$ have poles.

Next we define local derivatives $\partial_{2}$ and $\partial_{3}$ at the point $z$. We will be motivated by the requirements

$$
\begin{cases}\partial_{2} P_{01}^{(z)}(z)=\partial_{2} P_{03}^{(z)}(z)=0, & \partial_{2} P_{02}^{(z)}(z)=1, \\ \partial_{3} P_{01}^{(z)}(z)=\partial_{3} P_{02}^{(z)}(z)=0, & \partial_{3} P_{03}^{(z)}(z)=1 .\end{cases}
$$

Note that $N^{(5)}$ is orthogonal to $a^{(1)}$ and $a^{(3)}$, while $N^{(6)}$ is orthogonal to $a^{(1)}$ and $a^{(2)}$, since they are left and right eigenvectors for the same matrix. We normalize them so that $N^{(5)} \cdot a^{(2)}=N^{(6)} \cdot a^{(3)}=1$.

Definition 3.1. $\partial_{2}$ and $\partial_{3}$ are defined by

$$
\left\{\begin{array}{l}
\partial_{2} f(z)=\lim _{n \rightarrow \infty} \sigma_{2}^{-n} \sum_{i=0}^{2} N_{i}^{(5)} f\left(F_{w}^{n} q_{i}\right) \\
\partial_{3} f(z)=\lim _{n \rightarrow \infty} \sigma_{3}^{-n} \sum_{i=0}^{2} N_{i}^{(6)} f\left(F_{w}^{n} q_{i}\right)
\end{array}\right.
$$

provided the limits exist.

It is clear that (3.13) holds, and in fact it is not necessary to take the limit.

Theorem 3.2. Let $f \in \operatorname{dom} \Delta$. Then $\partial_{2} f(z)$ and $\partial_{3} f(z)$ exist.

Proof. We may write

$$
f(x)=h(x)+\int G(x, y) g(y) d \mu(y)
$$

for $h$ harmonic and $g$ continuous. Since $\partial_{2} h(z)$ and $\partial_{3} h(z)$ exist, it suffices to show that the local derivatives exist for the integral term in (3.15). At least formally, we expect

$$
\partial_{k} \int G(z, y) g(y) d \mu(y)=\int\left(\partial_{k} G(z, y)\right) g(y) d \mu(y),
$$

for $k=2,3$. For every $y \neq z, G(\cdot, y)$ is harmonic in a neighborhood of $z$, so $\partial_{k} G(z, y)$ is well defined. Moreover, it is a harmonic function of $y$ in $K \backslash\{z\}$, so it must be a linear combination of $P_{01}^{(z)}, P_{02}^{(z)}, \ldots, P_{06}^{(z)}$. Each of these functions is integrable, because the lower bound in (3.5) is strict, so the right side of (3.16) is well defined. For the left side, we observe that

$$
\begin{aligned}
& \sigma_{2}^{-n} \sum_{i=0}^{2} N_{i}^{(5)} \int G\left(F_{w}^{n} q_{i}, y\right) g(y) d \mu(y) \\
& =\sigma_{2}^{-n} \sum_{i=0}^{2} N_{i}^{(5)} \int_{K \backslash F_{w}^{n} K} G\left(F_{w}^{n} q_{i}, y\right) g(y) d \mu(y) \\
& \quad+\sigma_{2}^{-n} \sum_{i=0}^{2} N_{i}^{(5)} \int_{F_{w}^{n} K} G\left(F_{w}^{n} q_{i}, y\right) g(y) d \mu(y) \\
& =\int_{K \backslash F_{w}^{n} K} \partial_{2} G(z, y) g(y) d \mu(y)+\sigma_{2}^{-n} \sum_{i=0}^{2} N_{i}^{(5)} \int_{F_{w}^{n} K} G\left(F_{w}^{n} q_{i}, y\right) g(y) d \mu(y)
\end{aligned}
$$


because $G(\cdot, y)$ is harmonic in $F_{w}^{n} K$ for $y \in K \backslash F_{w}^{n} K$. So to prove (3.16) for $k=2$, we need

$$
\lim _{n \rightarrow \infty} \sigma_{2}^{-n} \sum_{i=0}^{2} N_{i}^{(5)} \int_{F_{w}^{n} K} G\left(F_{w}^{n} q_{i}, y\right) g(y) d \mu(y)=0 .
$$

This is easy, since $\sigma_{2}^{-n} \leq(5 / \sqrt{3})^{m n}$ and $\mu\left(F_{w}^{n} K\right)=\left(\frac{1}{3}\right)^{m n}$, with $\frac{5}{3 \sqrt{3}}<1$. But the same estimate will not work for $\sigma_{3}$ in place of $\sigma_{2}$. Instead we use a Hölder estimate on the Green's function, since $\sum_{i=1}^{3} N_{i}^{(6)}=0$. Note that $G\left(F_{w}^{n} q_{i}, y\right)-G\left(F_{w}^{n} q_{j}, y\right)$ is a sum of only $n m$ terms, and each is bounded by a constant times $\left(\frac{3}{5}\right)^{n m}$. So

$$
\begin{aligned}
\sigma_{3}^{-n} \sum_{i=0}^{2} N_{i}^{(6)} & \int_{F_{w} K} G\left(F_{w}^{n} q_{i}, y\right) g(y) d \mu(y) \\
& =\sigma_{3}^{-n} \sum_{i=0}^{2} N_{i}^{(6)} \int_{F_{w} K}\left(G\left(F_{w}^{n} q_{i}, y\right)-G\left(F_{w}^{n} q_{1}, y\right)\right) g(y) d \mu(y)
\end{aligned}
$$

is bounded by a multiple of

$$
\sigma_{3}^{-n} \cdot n m\left(\frac{3}{5}\right)^{n m} \cdot\left(\frac{1}{3}\right)^{n m}
$$

and this goes to zero as $n \rightarrow \infty$ by the strict lower bound in (3.5). So this proves (3.16) for $k=3$ also.

We can define "distributions" $\delta^{(z)}, \partial_{2} \delta^{(z)}$ and $\partial_{3} \delta^{(z)}$ supported at the point $z$ as bounded linear functionals on the test space $(\operatorname{dom} \Delta)_{00}$, the space of functions in $\operatorname{dom} \Delta$ vanishing in a neighborhood of the boundary by

$$
\left\langle\delta^{(z)}, \varphi\right\rangle=\varphi(z),\left\langle\partial_{k} \delta^{(z)}, \varphi\right\rangle=-\partial_{k} \varphi(z) \quad \text { for } k=2,3 .
$$

We can also define $\Delta P_{0 k}^{(z)}$ for $k=4,5,6$ in the "distribution sense" by

$$
\left\langle\Delta P_{0 k}^{(z)}, \varphi\right\rangle=\int P_{0 k}^{(z)} \Delta \varphi d \mu
$$

for $\varphi \in(\operatorname{dom} \Delta)_{00}$, since $P_{0 k}^{(z)}$ is integrable. Since $\Delta P_{0 k}^{(z)}=0$ in $K \backslash\{z\}$, the distributions $\Delta P_{0 k}^{(z)}$ will be supported at $z$, so we expect to be able to identify them with linear combinations of $\delta^{(z)}, \partial_{2} \delta^{(z)}$ and $\partial_{3} \delta^{(z)}$.

Theorem 3.3. There exist nonzero constants $c_{4}, c_{5}, c_{6}$ such that

$$
\Delta P_{04}^{(z)}=c_{4} \delta^{(z)}, \quad \Delta P_{05}^{(z)}=c_{5} \partial_{2} \delta^{(z)}, \quad \Delta P_{06}^{(z)}=c_{6} \partial_{3} \delta^{(z)} .
$$

Proof. Because $P_{0 k}^{(z)}$ is integrable, we have

$$
\int_{K} P_{0 k}^{(z)} \Delta \varphi d \mu=\lim _{n \rightarrow \infty} \int_{K \backslash F_{w}^{n} K} P_{0 k}^{(z)} \Delta \varphi d \mu .
$$

Because $\Delta P_{0 k}^{(z)}=0$ on $K \backslash F_{w}^{n} K$ we have

$$
\begin{aligned}
\int_{K \backslash F_{w}^{n} K} P_{0 k}^{(z)} \Delta \varphi d \mu & =\int_{K \backslash F_{w}^{n} K}\left(P_{0 k}^{(z)} \Delta \varphi-\varphi \Delta P_{0 k}^{(z)}\right) d \mu \\
& =\sum_{\partial F_{w}^{n} K}\left(\varphi \partial_{n} P_{0 k}^{(z)}-P_{0 k}^{(z)} \partial_{n} \varphi\right) .
\end{aligned}
$$


Here we have used the Gauss-Green formula (the sign change is due to the fact that the outward normals on $K \backslash F_{w}^{n} K$ become inward normals on $F_{w}^{n} K$ ) and the fact that $\varphi$ and $\partial_{n} \varphi$ vanish on $\partial K$. Thus

$$
\begin{aligned}
\int_{K} P_{0 k}^{(z)} \varphi d \mu=\lim _{n \rightarrow \infty}[ & \sigma_{k}^{n}\left(\frac{5}{3}\right)^{m n} N^{(k)} \cdot\left(\left.\varphi\right|_{\partial F_{w}^{n} K}\right) \\
& \left.+\sigma_{k}^{n}\left(\left(\frac{5}{3}\right)^{m n} D a^{(k)} \cdot\left(\left.\varphi\right|_{\partial F_{w}^{n} K}\right)-a^{(k)}\left(\left.\partial_{n} \varphi\right|_{\partial F_{w}^{n} K}\right)\right)\right] .
\end{aligned}
$$

Next we claim that the second term on the right side of (3.21) vanishes. Consider the difference $\varphi-\widetilde{h}$, where $\widetilde{h}$ is harmonic on $F_{w}^{n} K$ with $\left.\widetilde{h}\right|_{\partial F_{w}^{n} K}=\left.\varphi\right|_{\partial F_{w}^{n} K}$. We have $\Delta(\varphi-\widetilde{h})=\Delta \varphi$ on $F_{w}^{n} K$ and $\left.(\varphi-\widetilde{h})\right|_{\partial F_{w}^{n} K}=0$. Therefore

$$
\left|\sum_{\partial F_{w}^{n} K} h \partial_{n}(\varphi-\widetilde{h})\right|=\left|\int_{F_{w}^{n} K} h \Delta \varphi d \mu\right| \leq M\left(\frac{1}{3}\right)^{n m}
$$

for any harmonic function $h$ on $F_{w}^{n} K$. Of course, $\left.\partial_{n} \widetilde{h}\right|_{\partial F_{w}^{n} K}=\left.D \widetilde{h}\right|_{\partial F_{w}^{n} K}=\left.D \varphi\right|_{\partial F_{w}^{n} K}$. Thus

$$
\left(\frac{5}{3}\right)^{m n} \sigma_{k}^{n} a^{(k)} \cdot\left(\left.D \varphi\right|_{\partial F_{w}^{n} K}\right)-\sigma_{k}^{n} a^{(k)} \cdot\left(\left.\partial_{n} \varphi\right|_{\partial F_{w}^{n} K}\right)=O\left[\sigma_{k}^{n}\left(\frac{1}{3}\right)^{n m}\right],
$$

and this goes to zero by (3.5).

So in place of (3.21) we have

$$
\int_{K} P_{0 k}^{(z)} \varphi d \mu=\lim _{n \rightarrow \infty} \sigma_{k}^{n}\left(\frac{5}{3}\right)^{m n} N^{(k)} \cdot\left(\left.\varphi\right|_{\partial F_{w}^{n} K}\right) .
$$

When $k=5$ or 6 this gives (3.20) with $c_{5}=c_{6}=1$ in view of (3.14). When $k=4$, $\sigma_{k}^{n}\left(\frac{5}{3}\right)^{m n}$, so we have (3.20) with $c_{4}=N^{(4)} \cdot\left(\begin{array}{l}1 \\ 1 \\ 1\end{array}\right)$. We know this is not zero because $N^{(4)}$ is not zero and is orthogonal to $a^{(2)}$ and $a^{(3)}$.

Table 3.1 shows the values of $\sigma_{j}$ for some choices of $w$. Note that for $w=(0,1,2)$, $\sigma_{2}$ and $\sigma_{3}$ are complex conjugate pairs. Table 3.2 shows the values of $a^{(j)}$ for $j=1,2, \ldots, 6$ and $N^{(j)}$ for $j=4,5,6$ for the choice $w=(0,1)$.

We also observe that we can compute the distributional Laplacian of the harmonic functions with singularity at the junction point $F_{1} q_{2}$ shown in Figure 2.3. Since $\widetilde{h}_{4}$ is the Green's function,

$$
\Delta \widetilde{h}_{4}=\delta_{F_{1} q_{2}} .
$$

Next

$$
\begin{aligned}
\left\langle\Delta \widetilde{h}_{5}, v\right\rangle & =\int_{K} \widetilde{h}_{5} \Delta v d \mu \\
& =\sum_{i=0}^{2} \int_{F_{i} K} \widetilde{h}_{5} \Delta v d \mu \\
& =\sum_{i=0}^{2} \sum_{j=0}^{2} \widetilde{h}_{5}\left(F_{i} q_{j}\right) \partial_{n} v\left(F_{i} q_{j}\right)-\partial_{n} \widetilde{h}_{5}\left(F_{i} q_{j}\right) v\left(F_{i} q_{j}\right)
\end{aligned}
$$


since $\widetilde{h}_{5}$ is harmonic on each $F_{i} K$. But $v$ and $\partial_{n} v$ vanish at $q_{i}$ and the terms at $F_{0} q_{1}=F_{1} q_{0}$ and $F_{0} q_{2}=F_{2} q_{0}$ cancel, so

$$
\begin{aligned}
\left\langle\Delta \widetilde{h}_{5}, v\right\rangle= & \widetilde{h}_{5}\left(F_{1} q_{2}\right) \partial_{n} v\left(F_{1} q_{2}\right)+\widetilde{h}_{5}\left(F_{2} q_{1}\right) \partial_{n} v\left(F_{2} q_{1}\right) \\
& -\partial_{n} \widetilde{h}_{5}\left(F_{1} q_{2}\right) v\left(F_{1} q_{2}\right)-\partial_{n} \widetilde{h}_{5}\left(F_{2} q_{1}\right) v\left(F_{2} q_{1}\right) .
\end{aligned}
$$

Now $v\left(F_{2} q_{1}\right)=v\left(F_{1} q_{2}\right), \partial_{n} v\left(F_{2} q_{1}\right)=-\partial_{n} v\left(F_{1} q_{2}\right), \widetilde{h}_{5}\left(F_{1} q_{2}\right)=1, \widetilde{h}_{5}\left(F_{2} q_{1}\right)=-1$, $\partial_{n} \widetilde{h}_{5}\left(F_{1} q_{2}\right)=3$ and $\partial_{n} \widetilde{h}_{5}\left(F_{2} q_{1}\right)=-3$, so

$$
\left\langle\Delta \widetilde{h}_{5}, v\right\rangle=6 \partial_{n} v\left(F_{1} q_{2}\right)
$$

which we may write as

$$
\Delta \widetilde{h}_{5}=-6 \partial_{n} \delta_{F_{1} q_{2}} .
$$

For $\widetilde{h}_{6}$ and $\widetilde{h}_{7}$, we must interpret them as Cauchy principal integrals, since they are not integrable functions, but do possess cancellation near the pole. Let $U_{m}=F_{2} F_{1}^{m-1} K \cup F_{1} F_{2}^{m-1} K$ denote the standard neighborhood system around $F_{1} q_{2}$. Then

$$
\left\langle\widetilde{h}_{j}, v\right\rangle=\lim _{m \rightarrow \infty} \int_{K \backslash U_{m}} \widetilde{h}_{j} v d \mu, \quad j=6,7,
$$

is well defined for $v \in(\operatorname{dom} \Delta)_{00}$. We want to compute

$$
\left\langle\Delta \widetilde{h}_{j}, v\right\rangle=\left\langle\widetilde{h}_{j}, \Delta v\right\rangle=\lim _{m \rightarrow \infty} \int_{K \backslash U_{m}} \widetilde{h}_{j} \Delta v d \mu .
$$

Since $\widetilde{h}_{j}$ is harmonic on $K \backslash U_{m}$ we have

$$
\int_{K \backslash U_{m}} \widetilde{h}_{j} \Delta v d \mu=\sum_{\partial U_{m}}\left(v \partial_{n} \widetilde{h}_{j}-\widetilde{h}_{j} \partial_{n} v\right) .
$$

(The sign change occurs because the normal derivatives on the boundary of $U_{m}$ are the negatives of the normal derivatives on the boundary of $K \backslash U_{m}$.) Note that $\partial U_{m}$ consists of 4 points that we group into 2 pairs, $\left(F_{1} F_{2}^{m-1} q_{0}, F_{1} F_{2}^{m-1} q_{1}\right)$ and $\left(F_{2} F_{1}^{m-1} q_{0}, F_{2} F_{1}^{m-1} q_{2}\right)$, that we treat separately. The contribution to the right side of (3.27) coming from the first pair is

$$
\begin{aligned}
& v\left(F_{1} F_{2}^{m-1} q_{0}\right) \partial_{n} \widetilde{h}_{j}\left(F_{1} F_{2}^{m-1} q_{0}\right)+v\left(F_{1} F_{2}^{m-1} q_{1}\right) \partial_{n} \widetilde{h}_{j}\left(F_{1} F_{2}^{m-1} q_{1}\right) \\
- & \partial_{n} v\left(F_{1} F_{2}^{m-1} q_{0}\right) \widetilde{h}_{j}\left(F_{1} F_{2}^{m-1} q_{0}\right)-\partial_{n} v\left(F_{1} F_{2}^{m-1} q_{1}\right) \widetilde{h}_{j}\left(F_{1} F_{2}^{m-1} q_{1}\right) .
\end{aligned}
$$

When $j=6$, we have

$$
\widetilde{h}_{6}\left(F_{1} F_{2}^{m-1} q_{0}\right)=-\widetilde{h}_{6}\left(F_{1} F_{2}^{m-1} q_{1}\right)=3^{m-1}
$$

and

$$
\partial_{n} \widetilde{h}_{6}\left(F_{1} F_{2}^{m-1} q_{0}\right)=-\partial_{n} \widetilde{h}_{6}\left(F_{1} F_{2}^{m-1} q_{1}\right)=-\left(\frac{5}{9}\right) 5^{m},
$$

so (3.28) becomes

$$
\begin{aligned}
-\left(\frac{5}{9}\right) 5^{m}\left[v\left(F_{1} F_{2}^{m-1} q_{0}\right)-v\left(F_{1} F_{2}^{m-1} q_{1}\right)\right] & \\
& -\frac{1}{3} 3^{m}\left[\partial_{n} v\left(F_{1} F_{2}^{m-1} q_{0}\right)-\partial_{n} v\left(F_{1} F_{2}^{m-1} q_{1}\right)\right] .
\end{aligned}
$$


TABle 3.1.

\begin{tabular}{|c|c|c|c|c|c|c|}
\hline$w$ & $\sigma_{1}$ & $\sigma_{2}$ & $\sigma_{3}$ & $\sigma_{4}$ & $\sigma_{5}$ & $\sigma_{6}$ \\
\hline$(0,1)$ & 1 & $\begin{array}{l}\frac{7+\sqrt{13}}{50} \\
0.212111\end{array}$ & $\begin{array}{l}\frac{7-\sqrt{13}}{50} \\
0.0678889\end{array}$ & $\begin{array}{c}\frac{9}{25} \\
0.36\end{array}$ & $\begin{array}{l}\frac{7-\sqrt{13}}{2} \\
1.697244\end{array}$ & $\begin{array}{l}\frac{7+\sqrt{13}}{2} \\
5.3027756\end{array}$ \\
\hline$(0,1,1)$ & 1 & $\begin{array}{l}\frac{8+\sqrt{37}}{125} \\
0.112664\end{array}$ & $\begin{array}{l}\frac{8-\sqrt{37}}{125} \\
0.0153379\end{array}$ & $\begin{array}{c}\frac{27}{125} \\
0.216\end{array}$ & $\begin{array}{c}8-\sqrt{37} \\
1.9172375\end{array}$ & $\begin{array}{c}8+\sqrt{37} \\
14.082763\end{array}$ \\
\hline$(0,1,2)$ & 1 & $\begin{array}{r}\frac{5+\sqrt{2} i}{125} \\
\left|\sigma_{2}\right|= \\
0.0\end{array}$ & $\begin{array}{r}\frac{5-\sqrt{2} i}{125} \\
J_{3} \mid=\frac{\sqrt{27}}{125} \\
15692\end{array}$ & $\frac{27}{125}$ & $\begin{array}{r}5+\sqrt{2} i \\
\left|\sigma_{5}\right|=\mid c \\
5.19\end{array}$ & $\begin{array}{l}5-\sqrt{2} i \\
6 \mid=\sqrt{27} \\
1524\end{array}$ \\
\hline$(0,1,2,1)$ & 1 & $\begin{array}{l}\frac{11+2 \sqrt{10}}{625} \\
0.0277192\end{array}$ & $\begin{array}{c}\frac{11-2 \sqrt{10}}{625} \\
0.0074807115\end{array}$ & $\begin{array}{c}\frac{81}{625} \\
0.1296\end{array}$ & $\begin{array}{l}11-2 \sqrt{10} \\
4.6754447\end{array}$ & $\begin{array}{l}11+2 \sqrt{10} \\
17.3245553\end{array}$ \\
\hline$(0,0,1,1)$ & 1 & $\begin{array}{l}\frac{17+4 \sqrt{13}}{625} \\
0.0502755\end{array}$ & $\begin{array}{c}\frac{17-4 \sqrt{13}}{625} \\
0.0041244718\end{array}$ & $\begin{array}{c}\frac{81}{625} \\
0.1296\end{array}$ & $\begin{array}{l}17-4 \sqrt{13} \\
2.5777949\end{array}$ & $\begin{array}{l}17+4 \sqrt{13} \\
31.422205\end{array}$ \\
\hline$(0,0,0,1)$ & 1 & $\begin{array}{l}\frac{43+5 \sqrt{61}}{1250} \\
0.0656409\end{array}$ & $\begin{array}{c}\frac{43-5 \sqrt{61}}{1250} \\
0.0031590013\end{array}$ & $\begin{array}{c}\frac{81}{625} \\
0.1296\end{array}$ & $\begin{array}{l}\frac{43-5 \sqrt{61}}{2} \\
1.9743758\end{array}$ & $\begin{array}{l}\frac{43+5 \sqrt{61}}{2} \\
41.025624\end{array}$ \\
\hline
\end{tabular}

TABle 3.2. $w=(0,1)$

$$
\begin{gathered}
A_{1} A_{0}=\frac{1}{25}\left(\begin{array}{ccc}
16 & 5 & 4 \\
10 & 10 & 5 \\
13 & 6 & 6
\end{array}\right) \\
a^{(1)}=\left(\begin{array}{l}
1 \\
1 \\
1
\end{array}\right) \quad a^{(2)}=\left(\begin{array}{c}
25+7 \sqrt{13} \\
-55-15 \sqrt{13} \\
2
\end{array}\right) \quad a^{(3)}=\left(\begin{array}{c}
25-7 \sqrt{13} \\
-55+15 \sqrt{13} \\
2
\end{array}\right) \\
a^{(4)}=\frac{1}{81}\left(\begin{array}{l}
12 \\
20 \\
17
\end{array}\right) \quad a^{(5)}=\frac{1}{1548}\left(\begin{array}{c}
-71+311 \sqrt{13} \\
-662-40 \sqrt{13} \\
1243-361 \sqrt{13}
\end{array}\right) \quad a^{(6)}=\frac{1}{1548}\left(\begin{array}{c}
-71-311 \sqrt{13} \\
-662+40 \sqrt{13} \\
1243+361 \sqrt{13}
\end{array}\right) \\
N^{(4)}=\frac{1}{27}\left(\begin{array}{c}
15 \\
7 \\
5
\end{array}\right) \quad N^{(5)}=\left(\begin{array}{c}
1-\sqrt{13} \\
2 \\
-3+\sqrt{13}
\end{array}\right) \quad N^{(6)}=\left(\begin{array}{c}
1+\sqrt{13} \\
2 \\
-3-\sqrt{13}
\end{array}\right)
\end{gathered}
$$


Of course, $\lim _{m \rightarrow \infty} 5^{m}\left[v\left(F_{1} F_{2}^{m-1} q_{0}\right)-v\left(F_{1} F_{2}^{m-1} q_{1}\right)\right]=\partial_{T} v\left(F_{1} q_{2}\right)$ by definition. But also $\lim _{m \rightarrow \infty} 3^{m}\left[\partial_{n} v\left(F_{1} F_{2}^{m-1} q_{0}\right)-\partial_{n} v\left(F_{1} F_{2}^{m-1} q_{1}\right)\right]=\frac{3}{2} \partial_{T} v\left(F_{1} q_{2}\right)$. A similar analysis of the terms coming from the second pair produces the tangential derivative with respect to the other cell $F_{2} K$. Thus we have

$$
\Delta \widetilde{h}_{6}=c_{6}\left(\partial_{T} \delta_{F_{2} q_{1}}-\partial_{T} \delta_{F_{1} q_{2}}\right) .
$$

(This is actually symmetric with respect to $R_{0}$ because the definition of the two tangential derivatives is skew-symmetric.) A similar analysis yields

$$
\Delta \widetilde{h}_{7}=c_{7}\left(\partial_{T} \delta_{F_{2} q_{1}}+\partial_{T} \delta_{F_{1} q_{2}}\right) .
$$

\section{Monomials With Boundary POINT SINGUlarity}

In part I, we described a monomial basis $\left\{P_{j k}^{(0)}\right\}, j=0,1, \ldots, k=1,2,3$, for the space of polynomials, "centered" at the point $q_{0}$. In this section, we will describe another class of monomials, $\left\{P_{j 4}^{(0)}\right\}, j=0,1, \ldots$, that completes a basis for polynomials with singularity at $q_{0}$. We take $P_{04}^{(0)}=h_{4}$ described in section 2, and then we want

$$
\Delta P_{j 4}^{(0)}=P_{(j-1) 4}^{(0)} \quad \text { on } K \backslash\left\{q_{0}\right\} \text { for } j \geq 1 .
$$

We may interpret (4.1) either as saying

$$
\mathcal{E}\left(P_{j 4}^{(0)}, v\right)=-\int P_{(j-1) 4}^{(0)} v d \mu
$$

for all $v \in \operatorname{dom} \mathcal{E}$ vanishing at $q_{1}, q_{2}$ and in a neighborhood of $q_{0}$, or as

$$
P_{j 4}^{(0)}(x)=-\int G(x, y) P_{(j-1) 4}^{(0)}(y) d \mu(y)+h(x)
$$

for $h$ harmonic on $K \backslash\left\{q_{0}\right\}$. We note that although $P_{04}^{(0)}$ is not integrable, the product $G(x, y) P_{04}^{(0)}(y)$ is integrable because $G(x, y)$ vanishes as $y$ approaches $q_{0}$, so (4.3) exists as an ordinary integral. Of course, (4.1) does not uniquely determine $P_{j 4}^{(0)}$ for $j \geq 1$. To obtain uniqueness, we adjoin the scaling identity

$$
P_{j 4}^{(0)} \circ F_{0}^{m}=\left(\frac{3}{5^{j}}\right)^{m} P_{j 4}^{(0)} .
$$

Note that it suffices to have (4.4) hold for $m=1$, and then the general case follows by iteration.

Theorem 4.1. There exists a unique solution to (4.1) and (4.4).

Proof. We give an argument that allows us to effectively compute $P_{j 4}^{(0)}$. The idea is to find the restriction to $F_{2} K$. Because $P_{j 4}^{(0)}$ is skew-symmetric, this determines $P_{j 4}^{(0)}$ on $F_{1} K$ by odd reflection, and in fact (4.1) will continue to hold on $F_{1} K \cup F_{2} K$ if it holds on $F_{2} K$. Since this is an inductive argument, we begin with

$$
P_{04}^{(0)} \circ F_{2}=b_{0} P_{02}^{(1)}+c_{0} P_{03}^{(1)} .
$$

We know such an identity must hold because $P_{04}^{(0)}\left(F_{2} q_{1}\right)=0$ by the skew-symmetry, and $P_{04}^{(0)} \circ F_{2}$ is harmonic. Of course, we find $b_{0}=-4, c_{0}=-2$, because we know 
$P_{04}^{(0)}$ explicitly. Next, we write

$$
P_{14}^{(0)} \circ F_{2}=\frac{1}{5} b_{0} P_{12}^{(1)}+\frac{1}{5} c_{0} P_{13}^{(1)}+b_{1} P_{02}^{(1)}+c_{1} P_{03}^{(1)},
$$

since this is the most general solution of

$$
\Delta\left(P_{14}^{(0)} \circ F_{2}\right)=\frac{1}{5} P_{04}^{(0)}
$$

that vanishes at $q_{1}$. Iterating this argument, we arrive at the representation

$$
P_{j 4}^{(0)} \circ F_{2}=\sum_{k=0}^{j} 5^{k-j}\left(b_{k} P_{(j-k) 2}^{(1)}+c_{k} P_{(j-k) 3}^{(1)}\right)
$$

as the most general solution to (4.1) in $F_{2} K$ that vanishes at $F_{2} q_{1}$.

Next, we want to see how (4.4) inductively determines the coefficients. Note that (4.4) yields an extension from $F_{1} K \cup F_{2} K$ to $F_{0} F_{1} K \cup F_{0} F_{2} K$. We need to check the matching conditions, both for the value and normal derivatives at $F_{2} q_{0}=F_{0} q_{2}$ (the conditions will be the same at $F_{1} q_{0}=F_{0} q_{1}$ by skew-symmetry), as these will be necessary and sufficient for (4.1) to hold on $F_{1} K \cup F_{2} K \cup F_{0} F_{1} K \cup F_{0} F_{2} K$. Once we have solved this extension problem, we can inductively use (4.4) to extend $P_{j 4}^{(0)}$ to $\left(F_{0}\right)^{n} F_{1} K \cup\left(F_{0}\right)^{n} F_{2} K$, and the matching conditions will be the same.

The matching condition $P_{j 4}^{(0)} \circ F_{2}\left(q_{0}\right)=P_{j 4}^{(0)} \circ F_{0}\left(q_{2}\right)$, using (4.4), says

$$
P_{j 4}^{(0)} \circ F_{2} q_{0}=\frac{3}{5^{j}} P_{j 4}^{(0)} \circ F_{2}\left(q_{2}\right) .
$$

Using the data from part I, this becomes

$$
\sum_{k=0}^{j} 5^{k-j}\left(b_{k} \beta_{j-k}-c_{k} \gamma_{j-k}\right)=3 \sum_{k=0}^{j} 5^{k-2 j}\left(b_{k} \beta_{j-k}+c_{k} \gamma_{j-k}\right),
$$

which may be rewritten as

$$
\begin{aligned}
\frac{1}{2}\left(1-3.5^{-j}\right) b_{j} & +\frac{1}{2}\left(1+3.5^{-j}\right) c_{j} \\
= & \sum_{k=0}^{j-1}\left[\left(5^{k-j}-3.5^{k-2 j}\right) b_{k} \beta_{j-k}-\left(5^{k-j}+3.5^{k-2 j}\right) c_{k} \gamma_{j-k}\right]
\end{aligned}
$$

The matching condition for normal derivatives is

$$
\partial_{n}\left(P_{j 4}^{(0)} \circ F_{2}\right)\left(q_{0}\right)+\partial_{n}\left(P_{j 4}^{(0)} \circ F_{0}\right)\left(q_{2}\right)=0 .
$$

But

$$
\partial_{n}\left(P_{j 4}^{(0)} \circ F_{0}\right)\left(q_{2}\right)=3.5^{-j} \partial_{n} P_{j 4}^{(0)}\left(q_{2}\right)=5^{1-j} \partial_{n}\left(P_{j 4}^{(0)} \circ F_{2}\right)\left(q_{2}\right)
$$

by (4.4). Thus we obtain

$$
\begin{aligned}
\sum_{k=0}^{j} 5^{k-j}\left[\left(b_{k} \partial_{n} P_{(j-k) 2}^{(1)}\left(q_{0}\right)\right.\right. & \left.+c_{k} \partial_{n} P_{(j-k) 3}^{(1)}\left(q_{0}\right)\right) \\
& \left.+5^{1-j}\left(b_{k} \partial_{n} P_{(j-k) 2}^{(1)}\left(q_{2}\right)+c_{k} \partial_{n} P_{(j-k) 3}^{(1)}\left(q_{2}\right)\right)\right]=0 .
\end{aligned}
$$




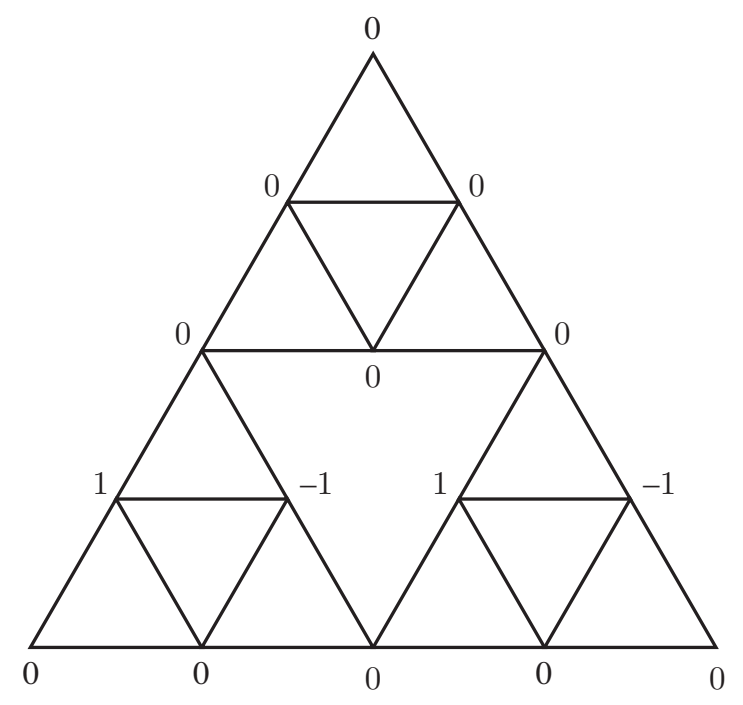

FIgURE 4.1. Values on $V_{2}$ of an eigenfunction with eigenvalue $5 \lambda_{0}^{(5)}$

Using data from part I, this becomes

$$
\begin{aligned}
\frac{1}{2}\left(1+5^{1-j}\right) b_{j} & +\frac{3}{2}\left(1-5^{1-j}\right) c_{j} \\
= & \sum_{k=0}^{j-1} 5^{k-j}\left[-\left(1+5^{1-j}\right) b_{k} \alpha_{j-k}+3\left(1-5^{1-j}\right) c_{k} n_{j-k+1}\right] .
\end{aligned}
$$

It is clear from inspection that (4.9) and (4.11) may be uniquely solved for $b_{j}$ and $c_{j}$ in terms of $b_{k}$ and $c_{k}$ for $k<j$, since

$$
\frac{1}{2}\left(1+5^{1-j}\right) \cdot \frac{1}{2}\left(1+3.5^{-j}\right)-\frac{3}{2}\left(1-5^{1-j}\right) \cdot \frac{1}{2}\left(1-3.5^{-j}\right) \neq 0 .
$$

This shows that (4.1) and (4.4) have a unique solution.

The values of $b_{j}$ and $c_{j}$ for small values of $j$ are displayed in Table 4.1. The data shows that both constants behave asymptotically like $\left(-5 \lambda_{0}^{(5)}\right)^{-j}$, where $5 \lambda_{0}^{(5)} \approx$ 279.4291373 is the eigenvalue generated by $\lambda_{2}=5$ and all $\varepsilon_{m}=-1$. The computations were done in rational arithmetic to avoid the possibility of instability in the algorithm. In Tables 4.2, 4.3 and 4.4, we display the values of $P_{j 4}^{(0)}$ at the points $F_{2} q_{0}, q_{2}, F_{2} F_{0} q_{1}, F_{2} F_{0} q_{2}$ and $F_{2} F_{2} q_{1}$. Again the eigenvalue $5 \lambda_{0}^{(5)}$ shows up in the values at $F_{2} F_{0} q_{1}$ and $F_{2} F_{0} q_{2}$, namely

$$
P_{j 4}^{(0)}\left(F_{2} F_{0} q_{1}\right) \approx-P_{j 4}^{(0)}\left(F_{2} F_{0} q_{2}\right) \approx c\left(-5 \lambda_{0}^{(5)}\right)^{-j} .
$$

The larger eigenvalues $\lambda_{0}^{(6)} \approx 677.8606$ (generated by $\lambda_{2}=6$ and all $\varepsilon_{m}=-1$ ) and $5 \lambda_{0}^{(6)}$ show up at $q_{2}, F_{2} F_{2} q_{1}$ and $F_{2} q_{0}$. We also observe the relationship $P_{j 4}^{(0)}\left(q_{2}\right) \approx$ $-2 P_{j 4}^{(0)}\left(F_{2} F_{2} q_{1}\right)$. At present, we have no explanation for this.

Conjecture 4.2. The limit as $j \rightarrow \infty$ of $\left(-5 \lambda_{0}^{(5)}\right)^{j} P_{j 4}^{(0)}$ exists and is a multiple of the eigenfunction with eigenvalue $5 \lambda_{0}^{(5)}$ shown in Figure 4.1. 
TABle 4.1.

\begin{tabular}{|c|c|c|c|c|}
\hline$j$ & $b_{j}$ & $c_{j}$ & $b_{j} / b_{j-1}$ & $c_{j} / c_{j-1}$ \\
\hline 0 & -4.0 & -2.0 & & \\
\hline 1 & 0.2666666667 & -0.03555555556 & -15.0 & 56.25000000 \\
\hline 2 & 0.01517037037 & -0.01512427984 & 17.57812500 & 2.350892468 \\
\hline 3 & 0.0001041086130 & -0.0002748737126 & 145.7167657 & 55.02264911 \\
\hline 4 & $2.225528997 \times 10^{-7}$ & $-1.244014207 \times 10^{-6}$ & 467.7926604 & 220.9570527 \\
\hline 5 & $2.669865631 \times 10^{-10}$ & $-2.094686014 \times 10^{-9}$ & 833.5734094 & 593.8905394 \\
\hline 6 & $7.435735448 \times 10^{-14}$ & $-1.972569325 \times 10^{-12}$ & 3590.587172 & 1061.907426 \\
\hline 7 & $3.340879006 \times 10^{-16}$ & $-6.947039102 \times 10^{-16}$ & 222.5682353 & 2839.438926 \\
\hline 8 & $-9.672270389 \times 10^{-19}$ & $-1.398455022 \times 10^{-18}$ & -345.4079416 & 496.7652867 \\
\hline 9 & $3.522400164 \times 10^{-21}$ & $3.649070802 \times 10^{-21}$ & -274.5931734 & -383.2359245 \\
\hline 10 & $-1.259441447 \times 10^{-23}$ & $-1.332178061 \times 10^{-23}$ & -279.6795494 & -273.9176472 \\
\hline 11 & $4.507418377 \times 10^{-26}$ & $4.754296157 \times 10^{-26}$ & -279.4152532 & -280.2051066 \\
\hline 12 & $-1.613085359 \times 10^{-28}$ & $-1.700385430 \times 10^{-28}$ & -279.4283857 & -279.6010877 \\
\hline 13 & $5.772796277 \times 10^{-31}$ & $6.083557903 \times 10^{-31}$ & -279.4287692 & -279.5050950 \\
\hline 14 & $-2.065925824 \times 10^{-33}$ & $-2.176896188 \times 10^{-33}$ & -279.4290196 & -279.4601753 \\
\hline 15 & $7.393381122 \times 10^{-36}$ & $7.790156743 \times 10^{-36}$ & -279.4290988 & -279.4418982 \\
\hline 16 & $-2.645887800 \times 10^{-38}$ & $-2.787830399 \times 10^{-38}$ & -279.4291248 & -279.4343854 \\
\hline 17 & $9.468904584 \times 10^{-41}$ & $9.976800851 \times 10^{-41}$ & -279.4291332 & -279.4312968 \\
\hline 18 & $-3.388660439 \times 10^{-43}$ & $-3.570411164 \times 10^{-43}$ & -279.4291359 & -279.4300262 \\
\hline 19 & $1.212708337 \times 10^{-45}$ & $1.277750245 \times 10^{-45}$ & -279.4291368 & -279.4295033 \\
\hline 20 & $-4.339949475 \times 10^{-48}$ & $-4.572714100 \times 10^{-48}$ & -279.4291371 & -279.4292880 \\
\hline 21 & $1.553148508 \times 10^{-50}$ & $1.636448199 \times 10^{-50}$ & -279.4291372 & -279.4291994 \\
\hline 22 & $-5.558291175 \times 10^{-53}$ & $-5.856397314 \times 10^{-53}$ & -279.4291373 & -279.4291629 \\
\hline 23 & $1.989159481 \times 10^{-55}$ & $2.095843386 \times 10^{-55}$ & -279.4291373 & -279.4291478 \\
\hline 24 & $-7.118654482 \times 10^{-58}$ & $-7.500446712 \times 10^{-58}$ & -279.4291373 & -279.4291416 \\
\hline 25 & $2.547570576 \times 10^{-60}$ & $2.684203493 \times 10^{-60}$ & -279.4291373 & -279.4291391 \\
\hline
\end{tabular}


TABle 4.2 .

\begin{tabular}{|c|c|c|c|c|}
\hline$j$ & $P_{j 4}^{(0)}\left(F_{2} q_{0}\right)$ & $\frac{P_{j 4}^{(0)}\left(F_{2} q_{0}\right)}{P_{(j-1) 4}^{(0)}\left(F_{2} q_{0}\right)}$ & $P_{j 4}^{(0)}\left(q_{2}\right)$ & $\frac{P_{j 4}^{(0)}\left(q_{2}\right)}{P_{(j-1) 4}^{(0)}\left(q_{2}\right)}$ \\
\hline 0 & 3 & & 1 & \\
\hline 1 & -0.07333333333 & -40.90909091 & -0.1222222222 & -8.181818183 \\
\hline 2 & -0.002098765432 & 34.94117647 & -0.01748971193 & 6.988235294 \\
\hline 3 & -0.000009252316072 & 226.8367634 & -0.0003855131697 & 45.36735267 \\
\hline 4 & $-1.592196961 \times 10^{-8}$ & 581.1037390 & $-3.317077001 \times 10^{-6}$ & 116.2207478 \\
\hline 5 & $-1.412709980 \times 10^{-11}$ & 1127.051542 & $-1.471572896 \times 10^{-8}$ & 225.4103083 \\
\hline 6 & $-7.469101008 \times 10^{-15}$ & 1891.405644 & $-3.890156775 \times 10^{-11}$ & 378.2811288 \\
\hline 7 & $-2.590083509 \times 10^{-18}$ & 2883.729803 & $-6.745009138 \times 10^{-14}$ & 576.7459607 \\
\hline 8 & $-6.289308978 \times 10^{-22}$ & 4118.232254 & $-8.189204398 \times 10^{-17}$ & 823.6464509 \\
\hline 9 & $-1.121385078 \times 10^{-25}$ & 5608.518520 & $-7.300684100 \times 10^{-20}$ & 1121.703704 \\
\hline 10 & $-1.521915038 \times 10^{-29}$ & 7368.250198 & $-4.954150514 \times 10^{-23}$ & 1473.650039 \\
\hline 11 & $-1.618487611 \times 10^{-33}$ & 9403.315958 & $-2.634257179 \times 10^{-26}$ & 1880.663192 \\
\hline 12 & $-1.379087423 \times 10^{-37}$ & 11735.93192 & $-1.122304218 \times 10^{-29}$ & 2347.186384 \\
\hline 13 & $-9.634433733 \times 10^{-42}$ & 14314.15132 & $-3.920261122 \times 10^{-33}$ & 2862.830263 \\
\hline 14 & $-5.513153728 \times 10^{-46}$ & 17475.35840 & $-1.121653997 \times 10^{-36}$ & 3495.071682 \\
\hline 15 & $-2.876372999 \times 10^{-50}$ & 19167.03338 & $-2.925997924 \times 10^{-40}$ & 3833.406674 \\
\hline 16 & $-6.533814579 \times 10^{-55}$ & 44022.87460 & $-3.323269948 \times 10^{-44}$ & 8804.574921 \\
\hline 17 & $-1.806057139 \times 10^{-58}$ & 3617.723071 & $-4.593040821 \times 10^{-47}$ & 723.5446140 \\
\hline 18 & $4.011288381 \times 10^{-62}$ & -4502.436543 & $5.100616939 \times 10^{-50}$ & -900.4873089 \\
\hline 19 & $-1.223998676 \times 10^{-65}$ & 3277.199935 & $-7.781974003 \times 10^{-53}$ & -655.4399870 \\
\hline 20 & $3.600440326 \times 10^{-69}$ & -3399.580510 & $1.144549156 \times 10^{-55}$ & -679.9161017 \\
\hline 21 & $-1.062555271 \times 10^{-72}$ & -3388.473451 & $-1.688886120 \times 10^{-58}$ & -677.6946903 \\
\hline 22 & $3.134970652 \times 10^{-76}$ & -3389.362737 & $2.491450828 \times 10^{-61}$ & -677.8725476 \\
\hline 23 & $-9.249612755 \times 10^{-80}$ & -3389.299352 & $-3.675465884 \times 10^{-64}$ & -677.8598705 \\
\hline 24 & $2.729060127 \times 10^{-83}$ & -3389.303396 & $5.422155315 \times 10^{-67}$ & -677.8606791 \\
\hline 25 & $-8.051979996 \times 10^{-87}$ & -3389.303163 & $-7.998923457 \times 10^{-70}$ & -677.8606326 \\
\hline
\end{tabular}


TABle 4.3 .

\begin{tabular}{|c|c|c|c|c|}
\hline$j$ & $P_{j 4}^{(0)}\left(F_{2} q_{1}\right)$ & $\frac{P_{j 4}^{(0)}\left(F_{2} q_{1}\right)}{P_{(j-1) 4}^{(0)}\left(F_{2} q_{1}\right)}$ & $P_{j 4}^{(0)}\left(F_{2} F_{0} q_{2}\right)$ & $\frac{P_{j 4}^{(0)}\left(F_{2} F_{0} q_{2}\right)}{P_{(j-1) 4}^{(0)}\left(F_{2} F_{0} q_{2}\right)}$ \\
\hline 0 & 1.4 & & 1.6 & \\
\hline 1 & -0.07191111111 & -19.46847960 & -0.09742222222 & -16.42335766 \\
\hline 2 & -0.003314396708 & 21.69659140 & -0.006677702058 & 14.58918373 \\
\hline 3 & -0.00001816503087 & 182.4602849 & -0.00007714925602 & 86.55562480 \\
\hline 4 & $-3.063788440 \times 10^{-8}$ & 592.8944255 & $-3.554734207 \times 10^{-7}$ & 217.0324180 \\
\hline 5 & $-3.143318796 \times 10^{-11}$ & 974.6986032 & $-8.120323792 \times 10^{-10}$ & 437.7576926 \\
\hline 6 & $-3.807810424 \times 10^{-15}$ & 8254.924605 & $-1.122276712 \times 10^{-12}$ & 723.5580765 \\
\hline 7 & $-4.442594124 \times 10^{-17}$ & 85.71141810 & $-9.513367661 \times 10^{-16}$ & 1179.683948 \\
\hline 8 & $1.383662940 \times 10^{-19}$ & -321.0748800 & $-7.568099567 \times 10^{-19}$ & 1257.035214 \\
\hline 9 & $-4.986994304 \times 10^{-22}$ & -277.4542852 & $2.163804580 \times 10^{-22}$ & -3497.589218 \\
\hline 10 & $1.782124292 \times 10^{-24}$ & -279.8342588 & $-1.880299126 \times 10^{-24}$ & -115.0776783 \\
\hline 11 & $-6.375286834 \times 10^{-27}$ & -279.5363312 & $6.348529025 \times 10^{-27}$ & -296.1787083 \\
\hline 12 & $2.281164104 \times 10^{-29}$ & -279.4751514 & $-2.281749050 \times 10^{-29}$ & -278.2308171 \\
\hline 13 & $-8.163109719 \times 10^{-32}$ & -279.4479289 & $8.163005180 \times 10^{-32}$ & -279.5231657 \\
\hline 14 & $2.921271755 \times 10^{-34}$ & -279.4368482 & $-2.921273307 \times 10^{-34}$ & -279.4331212 \\
\hline 15 & $-1.045430938 \times 10^{-36}$ & -279.4323038 & $1.045430919 \times 10^{-36}$ & -279.4324575 \\
\hline 16 & $3.741292262 \times 10^{-39}$ & -279.4304387 & $-3.741292264 \times 10^{-39}$ & -279.4304334 \\
\hline 17 & $-1.338902998 \times 10^{-41}$ & -279.4296725 & $1.338902998 \times 10^{-41}$ & -279.4296726 \\
\hline 18 & $4.791561667 \times 10^{-44}$ & -279.4293575 & $-4.791561667 \times 10^{-44}$ & -279.4293575 \\
\hline 19 & $-1.714767529 \times 10^{-46}$ & -279.4292279 & $1.714767529 \times 10^{-46}$ & -279.4292279 \\
\hline 20 & $6.136680364 \times 10^{-49}$ & -279.4291746 & $-6.136680364 \times 10^{-49}$ & -279.4291746 \\
\hline 21 & $-2.196148937 \times 10^{-51}$ & -279.4291526 & $2.196148937 \times 10^{-51}$ & -279.4291526 \\
\hline 22 & $7.859412616 \times 10^{-54}$ & -279.4291437 & $-7.859412616 \times 10^{-54}$ & -279.4291436 \\
\hline 23 & $-2.812667505 \times 10^{-56}$ & -279.4291398 & $2.812667505 \times 10^{-56}$ & -279.4291399 \\
\hline 24 & $1.006576308 \times 10^{-58}$ & -279.4291384 & $-1.006576308 \times 10^{-58}$ & -279.4291383 \\
\hline 25 & $-3.602259651 \times 10^{-61}$ & -279.4291377 & $3.602259651 \times 10^{-61}$ & -279.4291377 \\
\hline
\end{tabular}


TABle 4.4.

\begin{tabular}{|c|c|c|}
\hline$j$ & $P_{j_{4}}^{(0)}\left(F_{2} F_{2} q_{1}\right)$ & $\frac{P_{j_{4}}^{(0)}\left(F_{2} F_{2} q_{1}\right)}{P_{(j-1)}^{(0)}\left(F_{2} F_{2} q_{1}\right)}$ \\
\hline 0 & 1 & \\
\hline 1 & -0.079555555556 & -12.56983240 \\
\hline 2 & -0.006348971193 & 12.53046409 \\
\hline 3 & -0.00007717717964 & 82.26487704 \\
\hline 4 & $-3.545338022 \times 10^{-7}$ & 217.6863790 \\
\hline 5 & $-8.150707352 \times 10^{-10}$ & 434.9730482 \\
\hline 6 & $-1.111396727 \times 10^{-12}$ & 733.3751444 \\
\hline 7 & $-9.902175453 \times 10^{-16}$ & 1122.376323 \\
\hline 8 & $-6.177245608 \times 10^{-19}$ & 1603.008215 \\
\hline 9 & $-2.812999139 \times 10^{-22}$ & 2195.964273 \\
\hline 10 & $-9.931688197 \times 10^{-26}$ & 2832.347415 \\
\hline 11 & $-2.502488523 \times 10^{-29}$ & 3968.724774 \\
\hline 12 & $-8.400870671 \times 10^{-33}$ & 2978.844242 \\
\hline 13 & $2.718952861 \times 10^{-36}$ & -3089.744876 \\
\hline 14 & $-5.708435539 \times 10^{-39}$ & -476.3043819 \\
\hline 15 & $8.172891988 \times 10^{-42}$ & -698.4596820 \\
\hline 16 & $-1.208758569 \times 10^{-44}$ & -676.1393216 \\
\hline 17 & $1.782872299 \times 10^{-47}$ & -677.9838184 \\
\hline 18 & $-2.630175292 \times 10^{-50}$ & -677.8530330 \\
\hline 19 & $3.880109822 \times 10^{-53}$ & -677.8610433 \\
\hline 20 & $-5.724052603 \times 10^{-56}$ & -677.8606157 \\
\hline 21 & $8.444291203 \times 10^{-59}$ & -677.8606357 \\
\hline 22 & $-1.245726742 \times 10^{-61}$ & -677.8606349 \\
\hline 23 & $1.837732828 \times 10^{-64}$ & -677.8606351 \\
\hline 24 & $-2.711077666 \times 10^{-67}$ & -677.8606349 \\
\hline 25 & $3.999461728 \times 10^{-70}$ & -677.8606348 \\
\hline
\end{tabular}




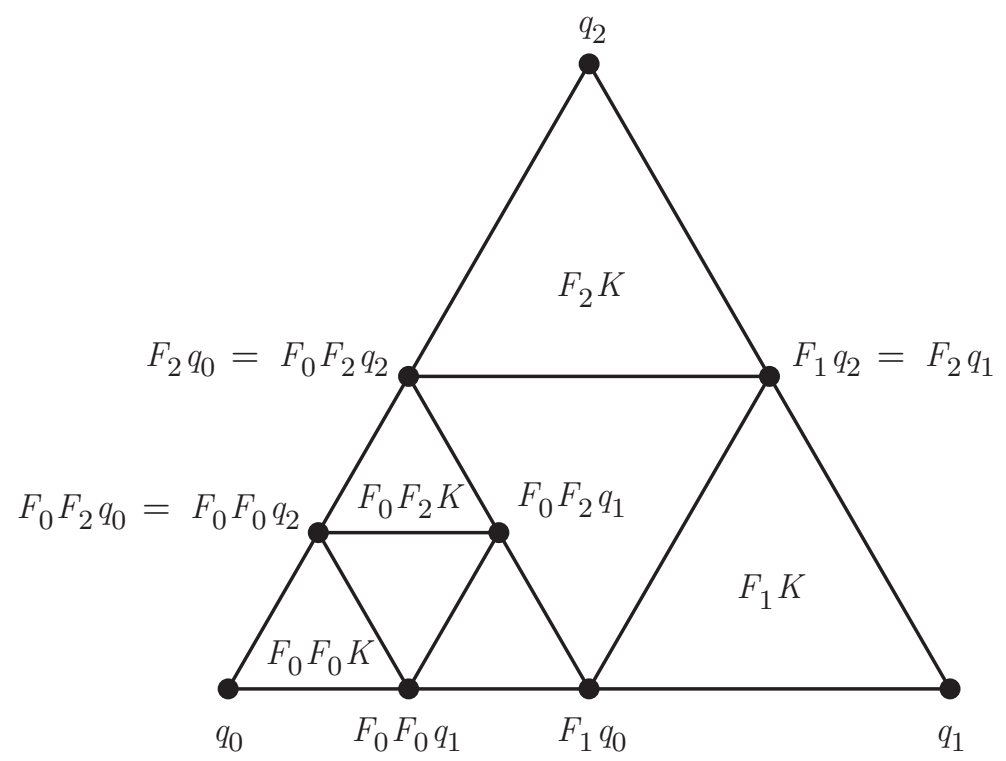

FIGURE 5.1.

\section{Monomials With PERIOdiC POINT SINGUlaRity}

Let $w$ and $z$ be as in section 3. We wish to extend the family $\left\{P_{0 k}^{(z)}\right\}, k=$ $1,2, \ldots, 6$, of harmonic functions on $K \backslash\{z\}$ to $\left\{P_{j k}^{(z)}\right\}$, for $j=0,1,2, \ldots$, of multiharmonic functions. We want

$$
\Delta P_{j k}^{(z)}=P_{(j-1) k}^{(z)} \quad \text { on } K \backslash\{z\}
$$

and

$$
P_{j k}^{(z)} \circ\left(F_{w}\right)^{n}=\left(5^{-j m} \sigma_{k}\right)^{n} P_{j k}^{(z)} .
$$

We will consider the case $w=(0,1)$. We want to keep track of the functions $P_{j k}^{(z)}$ on each of the cells $F_{1} K, F_{2} K, F_{02} K$ and $F_{00} K$ by expanding in bases of monomials centered at the points $F_{1} q_{2}, F_{2} q_{1}, F_{0} F_{2} q_{2}$ and $F_{0} F_{0} q_{2}$, respectively. (See Figure 5.1.) In other words,

$$
\begin{gathered}
P_{j k}^{(z)} \circ F_{1}=\sum_{l=0}^{j} \sum_{n=1}^{3} a_{l n k} 5^{l-j} P_{(j-l) n}^{(2)}, \\
P_{j k}^{(z)} \circ F_{2}=\sum_{l=0}^{j} \sum_{n=1}^{3} b_{l n k} 5^{l-j} P_{(j-l) n}^{(1)}, \\
P_{j k}^{(z)} \circ\left(F_{0} F_{2}\right)=\sum_{l=0}^{j} \sum_{n=1}^{3} c_{l n k} 5^{2(l-j)} P_{(j-l) n}^{(2)}, \\
P_{j k}^{(z)} \circ\left(F_{0} F_{0}\right)=\sum_{l=0}^{j} \sum_{n=1}^{3} d_{l n k} 5^{2(l-j)} P_{(j-l) n}^{(2)} .
\end{gathered}
$$


In order to have $\Delta^{k+1} P_{j k}^{(z)}=0$ in $K \backslash F_{0} F_{1} K$, we must have (5.3)-(5.6) and in addition matching conditions for the functions and normal derivatives at the points $F_{1} q_{2}=F_{2} q_{1}, F_{2} q_{0}=F_{0} F_{2} q_{2}$, and $F_{0} F_{2} q_{0}=F_{0} F_{0} q_{2}$. It might appear that the coefficients in (5.3)-(5.6) should also depend on $j$, but we have set up the righthand sides in such a way that if we have satisfied (5.1) for $j^{\prime}<j$, then we can reuse the same coefficients for $l<j$ and only have to determine new coefficients for $l=j$.

Since we have already determined $P_{0 k}^{(z)}$ in section 3 , we may compute the coefficients for $l=0$. Assume we have determined the coefficients for all $l<j$ so that (5.1) holds on $K \backslash F_{0} F_{1} K$. Then (5.1) for $l=j$ on $K \backslash F_{0} F_{1} K$ is automatic, provided we verify the matching conditions

$$
\begin{gathered}
\left\{\begin{array}{l}
P_{j k}^{(z)} \circ F_{1}\left(q_{2}\right)=P_{j k}^{(z)} \circ F_{2}\left(q_{1}\right), \\
\partial_{n}\left(P_{j k}^{(z)} \circ F_{1}\right)\left(q_{2}\right)=-\partial_{n}\left(P_{j k}^{(z)} \circ F_{2}\right)\left(q_{1}\right),
\end{array}\right. \\
\left\{\begin{array}{l}
P_{j k}^{(z)} \circ F_{2}\left(q_{0}\right)=P_{j k}^{(z)} \circ\left(F_{0} F_{2}\right)\left(q_{2}\right), \\
\frac{3}{5} \partial_{n}\left(P_{j k}^{(z)} \circ F_{2}\right)\left(q_{0}\right)=-\partial_{n}\left(P_{j k}^{(z)} \circ\left(F_{0} F_{2}\right)\right)\left(q_{2}\right),
\end{array}\right. \\
\left\{\begin{array}{l}
P_{j k}^{(z)} \circ\left(F_{0} F_{2}\right)\left(q_{0}\right)=P_{j k}^{(z)} \circ\left(F_{0} F_{0}\right)\left(q_{2}\right), \\
\partial_{n}\left(P_{j k}^{(z)} \circ\left(F_{0} F_{2}\right)\right)\left(q_{0}\right)=-\partial_{n}\left(P_{j k}^{(z)} \circ\left(F_{0} F_{0}\right)\right)\left(q_{2}\right) .
\end{array}\right.
\end{gathered}
$$

When we substitute (5.3)-(5.6) into (5.7)-(5.9), some of the expressions simplify because only one term is nonzero. Thus

$$
\left\{\begin{array}{cc}
P_{j k}^{(z)} \circ F_{1}\left(q_{2}\right)=a_{j 1 k}, & \partial_{n}\left(P_{j k}^{(z)} \circ F_{1}\right)\left(q_{2}\right)=a_{j 2 k}, \\
P_{j k}^{(z)} \circ F_{2}\left(q_{1}\right)=b_{j 1 k}, & \partial_{n}\left(P_{j k}^{(z)} \circ F_{2}\right)\left(q_{1}\right)=b_{j 2 k}, \\
P_{j k}^{(z)} \circ\left(F_{0} F_{2}\right)\left(q_{2}\right)=c_{j 1 k}, & \partial_{n}\left(P_{j k}^{(z)} \circ\left(F_{0} F_{2}\right)\right)\left(q_{2}\right)=c_{j 2 k}, \\
P_{j k}^{(z)} \circ\left(F_{0} F_{0}\right)\left(q_{2}\right)=d_{j 1 k}, & \partial_{n}\left(P_{j k}^{(z)} \circ\left(F_{0} F_{0}\right)\right)\left(q_{2}\right)=d_{j 2 k} .
\end{array}\right.
$$

However, the other four expressions do not simplify, and require the transplantation computations from part I. For the left side of (5.8) we need to use

$$
\begin{gathered}
P_{(j-l) n}^{(1)}\left(q_{0}\right)=P_{(j-l) n}^{(0)}\left(q_{2}\right)= \begin{cases}\alpha_{j-l} & \text { if } n=1, \\
\beta_{j-l} & \text { if } n=2, \\
-\gamma_{j-l} & \text { if } n=3,\end{cases} \\
\partial_{n} P_{(j-l) n}^{(1)}\left(q_{0}\right)=\partial_{n} P_{(j-l) n}^{(0)}\left(q_{2}\right)= \begin{cases}n_{j-l} & \text { if } n=1, \\
-\alpha_{j-l} & \text { if } n=2, l \neq j, \\
\frac{1}{2}-\alpha_{0} & \text { if } n=2, l=j, \\
-3 n_{j-l+1} & \text { if } n=3 .\end{cases}
\end{gathered}
$$


For the left side of (5.9), we need to use

$$
\begin{gathered}
P_{(j-l) n}^{(2)}\left(q_{0}\right)=P_{(j-l) n}^{(0)}\left(q_{1}\right)= \begin{cases}\alpha_{j-l} & \text { if } n=1, \\
\beta_{j-l} & \text { if } n=2, \\
\gamma_{j-l} & \text { if } n=3,\end{cases} \\
\partial_{n} P_{(j-l) n}^{(2)}\left(q_{0}\right)=\partial_{n} P_{(j-l) n}^{(0)}\left(q_{1}\right)= \begin{cases}n_{j-l} & \text { if } n=1, \\
-\alpha_{j-l} & \text { if } n=2, l \neq j, \\
\frac{1}{2}-\alpha_{0} & \text { if } n=2, l=j, \\
3 n_{j-l+1} & \text { if } n=3 .\end{cases}
\end{gathered}
$$

For fixed $k$ and $j,(5.7)-(5.9)$ give six linear equations in twelve unknowns, already explicitly solved for $a_{j 1 k}, a_{j 2 k}, c_{j 1 k}, c_{j 2 k}, d_{j 1 k}, d_{j 2 k}$, in terms of $a_{j 3 k}, b_{j 1 k}, b_{j 2 k}$, $b_{j 3 k}, c_{j 3 k}, d_{j 3 k}$, and earlier coefficients with $l<j$.

Now we assume that (5.2) has been established for all $j^{\prime}<j$. We then take (5.2) as a definition for extending $P_{j k}^{(z)}$ from $K \backslash F_{1} F_{1} K$ to $F_{0} F_{1} K \backslash\left(F_{0} F_{1}\right)^{2} K$, and then inductively from $\left(F_{0} F_{1}\right)^{n-1} K \backslash\left(F_{0} F_{1}\right)^{n} K$ to $\left(F_{0} F_{1}\right)^{n} K \backslash\left(F_{0} F_{1}\right)^{n+1} K$. This will of course guarantee that (5.2) holds, and (5.1) will hold on $K \backslash\{z\}$ provided matching conditions hold for each extension. But it suffices to establish this for $n=1$, at the points $F_{1} q_{0}=F_{0} F_{1} q_{1}, F_{0} F_{2} q_{1}=F_{0} F_{1} q_{2}$, and $F_{0} F_{0} q_{1}=F_{0} F_{1} q_{0}$. Specifically,

$$
\begin{gathered}
\left\{\begin{array}{l}
P_{j k}^{(z)} \circ F_{1}\left(q_{0}\right)=P_{j k}^{(z)} \circ\left(F_{0} F_{1}\right)\left(q_{1}\right) \\
=5^{-2 j} \sigma_{k} P_{j k}^{(z)} \circ F_{1}\left(q_{1}\right), \\
\frac{3}{5} \partial_{n}\left(P_{j k}^{(z)} \circ F_{1}\right)\left(q_{0}\right)=-\partial_{n}\left(P_{j k}^{(z)} \circ\left(F_{0} F_{1}\right)\right)\left(q_{1}\right) \\
=-\left(\frac{5}{3}\right) 5^{-2 j} \sigma_{k} \partial_{n}\left(P_{j k}^{(z)} \circ F_{1}\right)\left(q_{1}\right),
\end{array}\right. \\
\left\{\begin{array}{l}
P_{j k}^{(z)} \circ\left(F_{0} F_{2}\right)\left(q_{1}\right)=P_{j k}^{(z)} \circ\left(F_{0} F_{1}\right)\left(q_{2}\right) \\
=5^{-2 j} \sigma_{k} P_{j k}^{(z)} \circ F_{2}\left(q_{2}\right), \\
\partial_{n}\left(P_{j k}^{(z)} \circ\left(F_{0} F_{2}\right)\right)\left(q_{1}\right)=-\partial_{n}\left(P_{j k}^{(z)} \circ\left(F_{0} F_{1}\right)\right)\left(q_{2}\right) \\
=-\left(\frac{5}{3}\right) 5^{-2 j} \sigma_{k} \partial_{n}\left(P_{j k}^{(z)} \circ F_{2}\right)\left(q_{2}\right),
\end{array}\right. \\
\left\{\begin{array}{l}
P_{j k}^{(z)} \circ\left(F_{0} F_{0}\right)\left(q_{1}\right)=P_{j k}^{(z)} \circ\left(F_{0} F_{1}\right)\left(q_{0}\right) \\
=5^{-2 j} \sigma_{k} P_{j k}^{(z)} \circ\left(F_{0} F_{0}\right)\left(q_{0}\right), \\
\frac{3}{5} \partial_{n}\left(P_{j k}^{(z)} \circ\left(F_{0} F_{0}\right)\right)\left(q_{1}\right)=-\partial_{n}\left(P_{j k}^{(z)} \circ\left(F_{0} F_{1}\right)\right)\left(q_{0}\right) \\
=-\left(\frac{5}{3}\right)^{2} 5^{-2 j} \sigma_{k} \partial_{n}\left(P_{j k}^{(z)} \circ\left(F_{0} F_{0}\right)\right)\left(q_{0}\right),
\end{array}\right.
\end{gathered}
$$

where the first equality is the matching condition and the second equality is (5.2). Substituting (5.3)-(5.6) in (5.15)-(5.17) and using transplantation formulas analogous to (5.11)-(5.14), we obtain six more linear equations for the twelve coefficients with $l=j$.

Conjecture 5.1. The twelve linear equations in twelve unknowns have a unique solution, hence there is a unique solution to (5.1) and (5.2).

As evidence for the conjecture, we have numerically solved the equations for all $j \leq 15$. The numerical data exhibits an exponential decay given by the number 
$r=5.351906737 \ldots$. The evidence is that

$$
\lim _{j \rightarrow \infty} r^{j} P_{j k}^{(z)}
$$

exists for all $k=1,2, \ldots, 6$, and the limits are all multiples of a single function $u$, which satisfies the eigenvalue equation

$$
-\Delta u=-r u \quad \text { on } F_{1} K \cup F_{2} K .
$$

It is clear from (5.2) that $u$ must vanish on the boundary of $F_{0} F_{1} K$, but the evidence indicates that $u$ does not have vanishing normal derivatives at these points, so $u$ does not extend to an eigenfunction on $K$. Indeed, because the eigenvalue in (5.19) is negative, this would not be possible. Specifically, we found the values

$$
\left\{\begin{array}{cl}
u\left(q_{1}\right)=1, & u\left(F_{1} q_{2}\right)=2.650890858 \ldots, \\
u\left(q_{2}\right)=6.206573634 \ldots, & u\left(F_{2} q_{0}\right)=5.356932520 \ldots
\end{array}\right.
$$

Computing the discrete eigenvalue on level 1 at the point $F_{1} q_{2}$ from these values, we obtain $\lambda_{1}=-0.739352477$, which leads to $\lambda=-r$ if we choose all $\varepsilon_{j}=-1$.

These results are quite puzzling. It is not clear why the eigenvalue is negative, why the behavior of all six families is the same, and it is not clear what the significance of this particular eigenvalue and eigenfunction might be.

\section{Spectral Decimation With POINT Singularity}

To describe all eigenfunctions with "generic" eigenvalue we have the method of spectral decimation. In this section, we show how to modify the method to allow eigenfunctions with point singularity for the case of a boundary point. In principle, the same idea should work for any point singularity, but the details are more complicated.

Recall that the eigenvalue $\lambda$ may be written uniquely as

$$
\lambda=\lim _{m \rightarrow \infty} \frac{3}{2} 5^{m} \lambda_{m}
$$

where

$$
\lambda_{m}=\lambda_{m+1}\left(5-\lambda_{m+1}\right) .
$$

Of course, (6.2) means

$$
\lambda_{m+1}=\frac{5+\varepsilon_{m} \sqrt{25-4 \lambda_{m}}}{2}
$$

for $\varepsilon_{m}= \pm 1$, and $\varepsilon_{m}=-1$ for all but a finite number of $m$ 's, so that the limit (6.1) exists. Then $\lambda$ is called "generic" if no $\lambda_{m}$ equals 2,5 , or 6 . If $u$ is a $\lambda$-eigenfunction on any cell of level $m-1$, say $F_{w} K$, then the boundary values $u\left(F_{w} q_{i}\right)$ determine $u$ uniquely in the generic case, and in fact

$$
u\left(F_{w} F_{0} q_{1}\right)=\frac{4-\lambda_{m}}{\left(2-\lambda_{m}\right)\left(5-\lambda_{m}\right)}\left[u\left(F_{w} q_{0}\right)+u\left(F_{w} q_{1}\right)\right]+\frac{2}{\left(2-\lambda_{m}\right)\left(5-\lambda_{m}\right)} u\left(F_{w} q_{2}\right),
$$

and similar identities determine the boundary values of $u$ on the subcells $F_{w} F_{i} K$ of level $m$.

Now suppose we allow $u$ to have a singularity at $q_{0}$. Then there will be a 4 dimensional space of eigenfunctions in the generic case. Since we already know two global eigenfunctions that are $R_{0}$-symmetric and one that is skew-symmetric, we need to identify one more skew-symmetric eigenfunction on $K \backslash\left\{q_{0}\right\}$. Let us write 
$c_{j}=u\left(F_{0}^{j} q_{1}\right)=-u\left(F_{0}^{j} q_{2}\right)$. We will regard $c_{0}, c_{1}$ as "initial data" for $u$, and determine all the other $c_{j}$ values in terms of $c_{0}, c_{1}$ by a recursive formula.

Lemma 6.1. Let $u$ be an eigenfunction on $K \backslash\left\{q_{0}\right\}$ with generic eigenvalue, and assume $u$ is skew-symmetric with respect to $R_{0}$. Then

$$
\left(2-\lambda_{j}\right)\left[\left(5-\lambda_{j}\right) c_{j}-c_{j-1}\right]=\left(6-\lambda_{j}\right)\left[\left(5-\lambda_{j-1}\right) c_{j-1}-c_{j-2}\right] \quad \text { for } j \geq 2 .
$$

Moreover, $u$ has a removable singularity if and only if $\left(5-\lambda_{1}\right) c_{1}=c_{0}$, in which case

$$
c_{j-1}=\left(5-\lambda_{j}\right) c_{j} \quad \text { for all } j \geq 1 .
$$

Proof. By the skew-symmetry $u\left(F_{0}^{n} F_{1} q_{2}\right)=0$ for every $n$. Applying the spectral decimation method to $F_{1} K$ we find

$$
u\left(F_{1} F_{0} q_{1}\right)=\frac{\left(4-\lambda_{2}\right)\left(c_{0}+c_{1}\right)}{\left(2-\lambda_{2}\right)\left(5-\lambda_{2}\right)} \quad \text { and } \quad u\left(F_{1} F_{0} q_{2}\right)=\frac{\left(4-\lambda_{2}\right) c_{1}+2 c_{0}}{\left(2-\lambda_{2}\right)\left(5-\lambda_{2}\right)} .
$$

Thus the $\lambda_{2}$-eigenfunction equation on level 2 at $F_{0} q_{1}$ yields

$$
\left(4-\lambda_{2}\right) c_{1}=c_{2}+\frac{2\left(4-\lambda_{2}\right) c_{1}}{\left(2-\lambda_{2}\right)\left(5-\lambda_{2}\right)}+\frac{\left(6-\lambda_{2}\right) c_{0}}{\left(2-\lambda_{2}\right)\left(5-\lambda_{2}\right)} .
$$

Multiplying by $\left(2-\lambda_{2}\right)\left(5-\lambda_{2}\right)$ and rearranging terms yields

$$
\begin{aligned}
& \left(2-\lambda_{2}\right)\left[\left(5-\lambda_{2}\right) c_{2}-c_{1}\right]+\left(2-\lambda_{2}\right) c_{1}+2\left(4-\lambda_{2}\right) c_{1} \\
& =\left(6-\lambda_{2}\right)\left[\left(5-\lambda_{1}\right) c_{1}-c_{0}\right]+\left(2-\lambda_{2}\right)\left(5-\lambda_{2}\right)\left(4-\lambda_{2}\right) c_{1}-\left(6-\lambda_{2}\right)\left(5-\lambda_{1}\right) c_{1} .
\end{aligned}
$$

We may check that

$$
\left(2-\lambda_{2}\right)+2\left(4-\lambda_{2}\right)=\left(2-\lambda_{2}\right)\left(5-\lambda_{2}\right)\left(4-\lambda_{2}\right)-\left(6-\lambda_{2}\right)\left(5-\lambda_{1}\right)
$$

by using (6.2), so this establishes (6.5) for $j=2$. By iterating this argument, we obtain (6.5) for all $j \geq 2$. If $u$ has a removable singularity at $q_{0}$, then $u\left(q_{0}\right)=0$, and we may apply the $\lambda_{1}$-eigenvalue equation on level 1 at $F_{0} q_{1}$ to obtain $\left(5-\lambda_{1}\right) c_{1}=c_{0}$. Then (6.6) follows for all $j$ by (6.5).

To obtain a solution with a true singularity we have to take $\left(5-\lambda_{1}\right) c_{1} \neq c_{0}$. Let us write $\widetilde{c}_{j}=\left(5-\lambda_{j}\right) c_{j}-c_{j-1}$. We want to describe the asymptotic behavior of $\widetilde{c}_{j}$ and $c_{j}$. Note that it follows from (6.5) that

$$
\widetilde{c}_{j}=3^{j} \cdot \frac{1}{3}\left(\prod_{k=2}^{j} \frac{1-\frac{\lambda_{k}}{6}}{1-\frac{\lambda_{k}}{2}}\right) \widetilde{c}_{1} .
$$

Define the constant $A$ (depending on $\lambda$ ) by

$$
A=\frac{1}{3}\left(\prod_{k=2}^{\infty} \frac{1-\frac{\lambda_{k}}{6}}{1-\frac{\lambda_{k}}{2}}\right) \widetilde{c}_{1} .
$$

It is easy to see that the infinite product converges since

$$
\lambda_{k}=\frac{2 \lambda}{3} 5^{-k}+o\left(5^{-k}\right) \quad \text { as } k \rightarrow \infty
$$

by $(6.1)$. 
Theorem 6.2. We have the estimates

$$
\widetilde{c}_{j}=A 3^{j}-\frac{\lambda}{18} A\left(\frac{3}{5}\right)^{j}+o\left(\left(\frac{3}{5}\right)^{j}\right) \quad \text { as } j \rightarrow \infty
$$

and

$$
c_{j}=\frac{3}{14} A 3^{j}+\frac{11 \lambda}{420} A\left(\frac{3}{5}\right)^{j}+o\left(\left(\frac{3}{5}\right)^{j}\right) \quad \text { as } j \rightarrow \infty .
$$

Proof. We have exactly

$$
\widetilde{c}_{j}=A 3^{j} \prod_{k=j+1}^{\infty} \frac{1-\frac{\lambda_{k}}{2}}{1-\frac{\lambda_{k}}{6}}
$$

Let

$$
R_{j}=\prod_{k=j+1}^{\infty} \frac{1-\frac{\lambda_{k}}{2}}{1-\frac{\lambda_{k}}{6}}
$$

Then

$$
\log R_{j}=\sum_{k=j+1}^{\infty}\left[\log \left(1-\frac{\lambda_{k}}{2}\right)-\log \left(1-\frac{\lambda_{k}}{6}\right)\right]=-\frac{1}{3} \sum_{k=j+1}^{\infty} \lambda_{k}+O\left(5^{-2 j}\right) .
$$

Using (6.9) we obtain

$$
-\frac{1}{3} \sum_{k=j+1}^{\infty} \lambda_{k}=-\frac{2 \lambda}{9} \sum_{k=j+1}^{\infty} 5^{-k}+o\left(5^{-j}\right)=-\frac{\lambda}{18} 5^{-j}+o\left(5^{-j}\right) .
$$

This yields

$$
R_{j}=1-\frac{\lambda}{18} 5^{-j}+o\left(5^{-j}\right)
$$

and together with (6.12) we obtain (6.10).

If we assume that

$$
c_{j}=A_{1} 3^{j}+B_{1}\left(\frac{3}{5}\right)^{j}+o\left(\left(\frac{3}{5}\right)^{j}\right)
$$

and substitute this and (6.10) into the definition of $\widetilde{c}_{j}$ using (6.9), we obtain

$$
\begin{aligned}
A 3^{j}- & \frac{\lambda}{18} A\left(\frac{3}{5}\right)^{j}+o\left(\left(\frac{3}{5}\right)^{j}\right) \\
= & \left(5-\lambda_{j}\right)\left[A_{1} 3^{j}+B_{1}\left(\frac{3}{5}\right)^{j}+o\left(\left(\frac{3}{5}\right)^{j}\right)\right] \\
& \quad-\left[A_{1} 3^{j-1}+B_{1}\left(\frac{3}{5}\right)^{j-1}+o\left(\left(\frac{3}{5}\right)^{j}\right)\right] \\
= & \frac{14}{3} A_{1} 3^{j}+\left(\frac{10}{3} B_{1}-\frac{2 \lambda}{3} A_{1}\right)\left(\frac{3}{5}\right)^{j}+o\left(\left(\frac{3}{5}\right)^{j}\right) .
\end{aligned}
$$

This shows that

$$
A_{1}=\frac{3}{14} A \quad \text { and } \quad B_{1}=\frac{3}{10}\left(-\frac{\lambda}{18} A\right)+\frac{\lambda}{5} A_{1}=\frac{11 \lambda}{420} A .
$$


To actually show that (6.11) holds, we define the remainder $r_{j}$ by

$$
c_{j}=\frac{3}{14} A 3^{j}+\frac{11 \lambda}{420} A\left(\left(\frac{3}{5}\right)^{j}\right)+r_{j},
$$

and substitute this and (6.10) into the definition of $\widetilde{c}_{j}$. Using (6.9) this yields

$$
\left(5-\lambda_{j}\right) r_{j}-r_{j-1}=o\left(\left(\frac{3}{5}\right)^{j}\right) .
$$

It is a routine matter to show that (6.13) implies $r_{j}=o\left(\left(\frac{3}{5}\right)^{j}\right)$ because $\frac{1}{5}<\frac{3}{5}$.

An entirely different approach to the problem is to note that the general skewsymmetric eigenfunction on $K \backslash\left\{q_{0}\right\}$ can be expanded as

$$
u(x)=\alpha \sum_{k=0}^{\infty} \lambda^{k} P_{k 4}^{(0)}(x)+\beta \sum_{k=0}^{\infty} \lambda^{k} P_{k 3}^{(0)}(x)
$$

for constants $\alpha$ and $\beta$. This will converge in a neighborhood of $q_{0}$. (The size of the neighborhood depends on $\lambda$.) As long as $\alpha \neq 0$ the singularity will not be removable. It is clear from (6.14) that (6.11) is just the beginning of an infinite asymptotic expansion involving terms of order $\left(3 \cdot 5^{-l}\right)^{j}$ for $l=0,1, \ldots$ and $5^{-l j}$ for $l=1,2, \ldots$. (If $\beta=0$, these terms will be absent.)

\section{Generic eigenfunctions on BLOW-UpS}

Fix an infinite word $w$, and consider the blow-up

$$
\mathrm{SG}_{w}=\bigcup_{n=0}^{\infty} F_{w_{1}}^{-1} F_{w_{2}}^{-1} \cdots F_{w_{n}}^{-1} K \quad \text { (increasing union). }
$$

(See [S1.) If all $w_{i}$ are equal, say $w_{i}=0$ (or all but a finite number), then the blow-up contains a boundary point $\left(q_{0}\right)$. If all $w_{i}$ omit a value, say $w_{i}=0$ or 1 (or all but a finite number), then the blow-up contains a "bottom line" (the line joining $q_{0}$ and $q_{1}$ ). In [T1, Teplyaev has identified all $L^{2}$ eigenfunctions on all blowups. In part I, we studied generic eigenfunctions on the blow-up with boundary point. In this section, we study generic eigenfunctions on all blow-ups. We will pay attention to the "behavior at infinity" of the eigenfunctions, more precisely in the three different "directions to infinity."

We begin with an observation about the blow-up studied in part I that extends to all bottom line blow-ups. In the notation of part I, we defined a basis $C_{-\lambda}$, $S_{-\lambda}$ and $Q_{-\lambda}$ for the eigenspace $-\Delta u=\lambda u$ for $\lambda$ negative, and also a decaying eigenfunction $E_{-\lambda}=C_{-\lambda}-S_{-\lambda}$. Actually, the definitions are valid for any generic eigenvalue, but the asymptotic properties depend on $\left|\lambda_{j}\right| \rightarrow \infty$ as $j \rightarrow-\infty$. This condition is equivalent to the statement that $\lambda_{0}$ does not belong to the Julia set $J$ for the polynomial $z(5-z)$. This Julia set is a subset of the real axis interval $[0,5]$. We will refer to such eigenvalues as non-Julia generic.

Definition 7.1. The level eigenfunction $L_{-\lambda}$ is defined to be

$$
L_{-\lambda}(x)=C_{-\lambda}(x)+\frac{\lambda}{3} Q_{-\lambda}(x) .
$$

Theorem 7.2. $L_{-\lambda}(x)=1$ along the bottom line. 
Proof. From (6.15) and (6.18) in part I, we have $C_{-\lambda}\left(x_{n}\right)=1-\frac{\lambda_{n}}{4}$ and $Q_{-\lambda}\left(x_{n}\right)=$ $\frac{3 \lambda_{n}}{4 \lambda}$, where $x_{n}=F_{0}^{n} q_{1}$ and $n \leq 0$, so $L_{-\lambda}\left(x_{n}\right)=1$. But the same argument works for $n \geq 1$. In fact, we know more. Since $L_{-\lambda}\left(q_{0}\right)=L_{-\lambda}\left(q_{1}\right)=1, L_{-\lambda}$ restricted to $K$ must be symmetric with respect to the symmetry $R_{2}$ that interchanges $q_{0}$ and $q_{1}$. In particular, $L_{-\lambda}\left(F_{1} F_{0} q_{1}\right)=L_{-\lambda}\left(F_{0} F_{0} q_{1}\right)=1$. By similar reasoning, we find that $L_{-\lambda}(x)=1$ for every junction point on the bottom line, and since these are dense in the line, the result follows.

It also follows from (7.2) that

$$
L_{-\lambda}\left(F_{0}^{n} q_{2}\right)=\left(1-\frac{\lambda_{n}}{4}\right)-\frac{\lambda_{n}}{4}=1-\frac{\lambda_{n}}{2}
$$

because $C_{-\lambda}$ is symmetric and $Q_{-\lambda}$ is skew-symmetric with respect to the $R_{0}$ symmetry. This function also has a "translational" symmetry: if $C$ denotes any cell of level $n$ that lies along the bottom line, then $L_{-\lambda}$ is equal to 1 at the two vertices of $C$ that lie on the bottom line, and is equal to $1-\frac{\lambda_{n}}{2}$ at the third vertex of $C$. The values inside $C$ are then determined by spectral decimation. Note that this description makes sense for any blow-up that has a bottom line. The function $L_{-\lambda}$ is not bounded for a non-Julia generic eigenvalue, since $\lambda_{n}$ is unbounded as $n \rightarrow \infty$.

Next, we describe eigenfunctions that decay in two directions and blow up in the third direction, namely along the sequences $F_{w_{1}}^{-1} F_{w_{2}}^{-1} \cdots F_{w_{n}}^{-1} q_{i}$ as $n \rightarrow \infty$ for fixed $i$. For this we will require a genericity condition on the blow-up. We say that $w$ is generic in direction $i$ if there exists $N$ such that for every $j$, the segment $w_{j+1}, w_{j+2}, \ldots, w_{j+N}$ of length $N$ contains the digit $i$.

Lemma 7.3. For $w$ generic in direction 0 ,

$$
\lim _{m \rightarrow \infty} \frac{E_{-\lambda}\left(F_{0}^{-m} F_{w_{m}} F_{w_{m-1}} \cdots F_{w_{1}} x\right)}{E_{-\lambda}\left(F_{0}^{-m} F_{w_{m}} F_{w_{m-1}} \cdots F_{w_{1}} q_{0}\right)}
$$

exists uniformly on compact sets, for non-Julia generic eigenvalues. The limit, which we denote by $E_{-\lambda}^{(0)}(x)$, is a $\lambda$-eigenfunction.

Proof. For $x$ in a compact subset of $\mathrm{SG}_{w}$ we have $F_{w_{m}} F_{w_{m-1}} \cdots F_{w_{1}} x \in K$ for $m$ large enough, so

$$
a_{m}=\frac{E_{-\lambda}\left(F_{0}^{-m} F_{w_{m}} F_{w_{m-1}} \cdots F_{w_{1}} x\right)}{E_{-\lambda}\left(F_{0}^{-m} F_{w_{m}} F_{w_{m-1}} \cdots F_{w_{1}} q_{0}\right)}
$$

is well defined. We need to compare $a_{m+1}$ and $a_{m}$. Note that

$$
E_{-\lambda}\left(F_{0}^{-m} x\right)=E_{-5^{m} \lambda}(x) .
$$

Indeed, both sides of (7.4) are $5^{m} \lambda$-eigenfunctions that are equal to 1 at $x=q_{0}$ and have limit zero as $n \rightarrow \infty$ for $x=F_{0}^{-n} q_{i}$ for $i=1,2$, and such a function is unique. Thus we have

$$
a_{m}=\frac{E_{-5^{m} \lambda}\left(z_{m}\right)}{E_{-5^{m} \lambda}\left(z_{m}^{\prime}\right)}
$$

for $z_{m}=F_{w_{m}} F_{w_{m-1}} \cdots F_{w_{1}} x$ and $z_{m}^{\prime}=F_{w_{m}} F_{w_{m-1}} \cdots F_{w_{1}} q_{0}$. If $w_{m+1}=0$, then $a_{m+1}=a_{m}$. If $w_{m+1}=1$ (a similar analysis holds if $w_{m+1}=2$ ), then

$$
a_{m+1}=\frac{E_{-5^{m} \lambda}\left(F_{0}^{-1} F_{1} z_{m}\right)}{E_{-5^{m} \lambda}\left(F_{0}^{-1} F_{1} z_{m}^{\prime}\right)} .
$$


Now we claim that

$$
E_{-5^{m} \lambda}\left(F_{0}^{-1} F_{1} z\right)=-\frac{1}{\lambda_{-m}} E_{-5^{m} \lambda}(z)+O\left(\frac{1}{\lambda_{-m-1}}\right)
$$

for $z \in K$. Also note that

$$
\frac{1}{\lambda_{-m-1}}=-\left(\frac{1}{\lambda_{-m}}\right)^{2}+O\left(\frac{1}{\lambda_{-m-2}}\right)
$$

for large $m$ and $\lambda_{0} \notin J$. First we verify (7.7) at the boundary points. We know $E_{-5^{m} \lambda}\left(q_{0}\right)=1$ and $E_{-5^{m} \lambda}\left(q_{1}\right)=E_{-5^{m} \lambda}\left(q_{2}\right)=-\frac{1}{\lambda_{-m}}+O\left(\frac{1}{\lambda_{-m-1}}\right)$ while $E_{-5^{m} \lambda}\left(F_{0}^{-1} q_{1}\right)=E_{-5^{m} \lambda}\left(F_{0}^{-1} q_{2}\right)=-\frac{1}{\lambda_{-m-1}}+O\left(\frac{1}{\lambda_{-m-2}}\right)$ by Theorem 6.5 of part I. Then by spectral decimation $E_{-5^{m} \lambda}\left(F_{0}^{-1} F_{1} q_{2}\right)=-\frac{2}{\lambda_{-m-1}}+O\left(\frac{1}{\lambda_{-m-2}}\right)$. Thus

$$
\begin{aligned}
-\frac{1}{\lambda_{-m}}+O\left(\frac{1}{\lambda_{-m-1}}\right)=E_{-5^{m} \lambda}\left(q_{1}\right) & =E_{-5^{m} \lambda}\left(F_{0}^{-1} F_{1} q_{0}\right) \\
& =-\frac{1}{\lambda_{-m}} E_{-5^{m} \lambda}\left(q_{0}\right)+O\left(\frac{1}{\lambda_{-m-1}}\right), \\
-\frac{1}{\lambda_{-m-1}}+O\left(\frac{1}{\lambda_{-m-2}}\right)=E_{-5^{m} \lambda}\left(F_{0}^{-1} q_{1}\right) & =E_{-5^{m} \lambda}\left(F_{0}^{-1} F_{1} q_{1}\right) \\
& =-\frac{1}{\lambda_{-m}} E_{-5^{m} \lambda}\left(q_{1}\right)+O\left(\frac{1}{\lambda_{-m-2}}\right),
\end{aligned}
$$

and

$$
\begin{aligned}
-\frac{2}{\lambda_{-m-1}}+O\left(\frac{1}{\lambda_{-m-2}}\right) & =E_{-5^{m} \lambda}\left(F_{0}^{-1} F_{1} q_{2}\right) \\
& =-\frac{1}{\lambda_{-m}} E_{-5^{m} \lambda}\left(q_{2}\right)+O\left(\frac{1}{\lambda_{-m-1}}\right) .
\end{aligned}
$$

This verifies (7.7) at boundary points of $K$, and it follows for all points by spectral decimation, since the cumulative multiplication factor for the error is bounded by

$$
\prod_{j=-\infty}^{\infty}\left(\frac{2\left|4-\lambda_{j}\right|+2}{\left|2-\lambda_{j}\right|\left|5-\lambda_{j}\right|}\right),
$$

which depends only on $\lambda$.

Using (7.7) for $z=z_{m}$ and $z_{m}^{\prime}$ in (7.6) yields

$$
a_{m+1}=a_{m}+\frac{O\left(\frac{1}{\lambda_{-m}}\right)}{E_{-5^{m} \lambda}\left(z_{m}^{\prime}\right)} .
$$

Thus to establish the existence of the limit in (7.3) we need to show that

$$
\sum_{m=1}^{\infty} \frac{O\left(\frac{1}{\lambda_{-m}}\right)}{\left|E_{-5^{m} \lambda}\left(z_{m}^{\prime}\right)\right|}
$$


is finite. Under the generic assumption, the points $z_{m}^{\prime}$ for large $m$ are confined to a subset of $K$ of the form $\bigcup F_{w^{\prime}} K$, where each $w^{\prime}$ is of length $N$ and contains the digit 0 . On such a subset we have an upper bound for $\left(\left|E_{-5^{m} \lambda}(z)\right|\left|\lambda_{-m}\right|\right)^{-1}$ that decays exponentially, so (7.8) converges.

We could obtain the same conclusion under somewhat weaker genericity assumptions, but it is not clear whether such assumptions can be dispensed with entirely.

We may similarly define eigenfunctions $E_{-\lambda}^{(1)}(x)$ and $E_{-\lambda}^{(2)}(x)$ if $w$ is generic in direction 1 or 2 by permuting the boundary points.

In order to describe the decay of these eigenfunctions we introduce the notion of a chain of cells of fixed level $l$. The chain $\mathcal{C}$ is a sequence $\left\{C_{n}\right\}$ of cells that may be finite, infinite in one direction, or infinite in both directions, such that consecutive cells intersect at a single point. A finite chain is called a cycle if the first and last cells also intersect at a single point. If we fix the initial cell $C_{1}$, then there are three canonical infinite chains that pass through the points $F_{w_{1}}^{-1} F_{w_{2}}^{-1} \cdots F_{w_{m}}^{-1} q_{i}$ for fixed $i$, for all large $m$. (Here we only need to assume that the blow-up does not have a boundary point.) Indeed, if we have a finite chain $\mathcal{C}_{m}$ connecting $\mathcal{C}_{1}$ to a cell containing $F_{w_{1}}^{-1} F_{w_{2}}^{-1} \cdots F_{w_{m}}^{-1} q_{i}$, we may continue it to a chain $\mathcal{C}_{m+1}$ by traveling in a straight line from $F_{w_{1}}^{-1} F_{w_{2}}^{-1} \cdots F_{w_{m}}^{-1} q_{i}$ to $F_{w_{1}}^{-1} F_{w_{2}}^{-1} \cdots F_{w_{m}}^{-1} F_{w_{m+1}}^{-1} q_{i}$ along one side of the cell $F_{w_{1}}^{-1} F_{w_{2}}^{-1} \cdots F_{w_{m+1}}^{-1} F_{i} K$. (If $w_{m+1}=i$, then take $\mathcal{C}_{m+1}=\mathcal{C}_{m}$.) Then the chain $\mathcal{C}^{(i)}$ is the union of all $\mathcal{C}_{m}$.

Theorem 7.4. Assume $w$ is generic in direction 0 . Then $E_{-\lambda}^{(0)}$ vanishes at infinity along the chains $\mathcal{C}^{(1)}$ and $\mathcal{C}^{(2)}$, and is unbounded along the chain $\mathcal{C}^{(0)}$.

Proof. Consider the cell $F_{w_{1}}^{-1} F_{w_{2}}^{-1} \cdots F_{w_{m+1}}^{-1} K$ for large $m$, and the portion of the chain $\mathcal{C}^{(1)}$ or $\mathcal{C}^{(2)}$ that lies along the line connecting $F_{w_{1}}^{-1} F_{w_{2}}^{-1} \cdots F_{w_{m}}^{-1} q_{i}$ to $F_{w_{1}}^{-1} F_{w_{2}}^{-1}$ $\cdots F_{w_{m}}^{-1} F_{w_{m+1}}^{-1} q_{i}$. Using (7.5) we see that $E_{-\lambda}^{(0)}(x)$ is the ratio of $E_{-5^{m} \lambda}\left(z_{m}\right)$ with $z_{m}$ lying in $F_{0}^{-1} F_{1} K \cup F_{0}^{-1} F_{2} K$, to $E_{-5^{m} \lambda}\left(z_{m}^{\prime}\right)$ with $z_{m}^{\prime}$ lying in a subset of $K$ as described in Lemma 7.3. Since $\left|E_{-5^{m} \lambda}\left(z_{m}\right)\right|=O\left(\frac{1}{\lambda_{-m}}\right)$ we obtain the desired decay. On the other hand, the chain $\mathcal{C}^{(0)}$ contains the points $F_{w_{1}}^{-1} F_{w_{2}}^{-1} \cdots F_{w_{m}}^{-1} q_{0}$, where the numerator in (7.5) is 1 and the denominator tends to 0 . So the ratio is unbounded.

We can describe the rate of decay in quantitative terms. To do this we need to observe that $E_{-5^{m} \lambda}$ on a cell $F_{w^{\prime}} K$ of order $N$, where at least one digit of $w^{\prime}$ is 1 , is bounded below by a multiple of $\frac{1}{\left|\lambda_{-m}\right|^{1-2^{-N}}}$. (This may be established by induction using spectral decimation.) Thus the rate of decay is $\left|\lambda_{-m}\right|^{-2^{-N}}$.

If $w$ is generic in all three directions, then we have a basis $E_{-\lambda}^{(0)}, E_{-\lambda}^{(1)}, E_{-\lambda}^{(2)}$ for the space of eigenfunctions, each decaying along two of the three chains $\mathcal{C}^{(i)}$. On the other hand, if the blow-up has a bottom line (say $w_{i} \neq 2$ ) and $w$ is generic in the directions 0 and 1, then $E_{-\lambda}^{(0)}, E_{-\lambda}^{(1)}$ and $L_{-\lambda}$ form a basis.

In Figure 7.1, we show a typical graph of $E_{-\lambda}^{(0)}$. By taking a large value for $\lambda$ $\left(\lambda_{0}=10000\right)$, we can see the global behavior by taking the restriction to $K$. We also show the logarithm of $E_{-\lambda}^{(0)}$ in order to clarify the behavior where the function assumes values close to zero. In Figure 7.2, we restrict this function to two lines in $K$. The restriction to the boundary line joining $q_{0}$ and $q_{1}$ shows the expected 

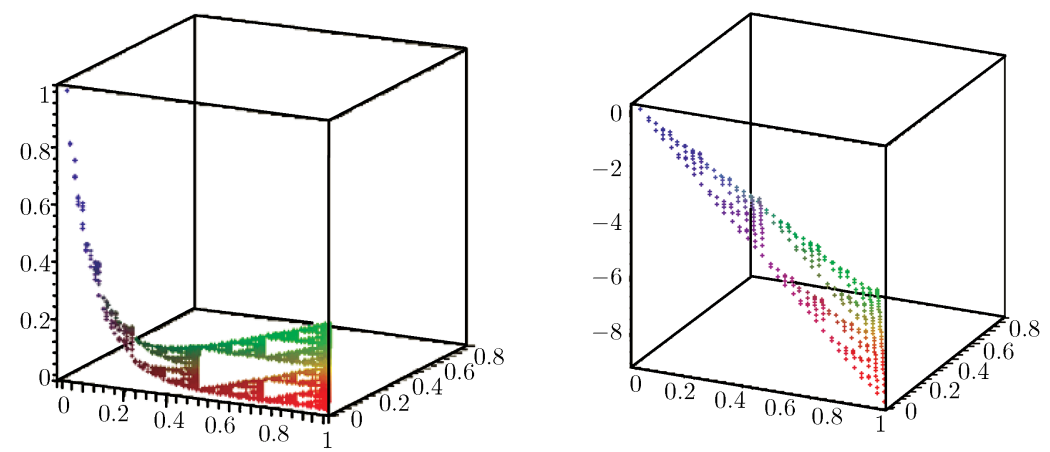

Figure 7.1. The graph of $E_{-\lambda}^{(0)}$ (left) and $\log E_{-\lambda}^{(0)}$ (right) on $K$ for $\lambda_{0}=10000$.
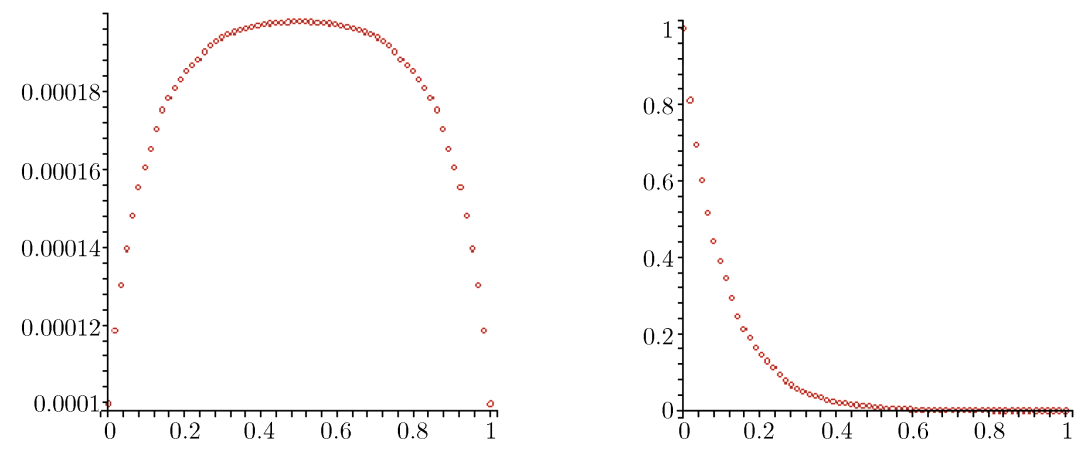

Figure 7.2. Restrictions of the function in Figure 7.1 to the line joining $F_{0} q_{1}$ and $F_{0} q_{2}$ (left) and the line joining $q_{0}$ and $q_{1}$ (right).

exponential decay. The restriction to the line joining $F_{0} q_{1}$ and $F_{0} q_{2}$ suggests that the limiting behavior as $\lambda \rightarrow \infty$ (rescaled by multiplying the function by $\lambda_{0}$ ) will be a discontinuous function equal to 1 at the endpoints and 2 on the interior of the line segment.

Finally, for the blow-up with boundary point $q_{0}$, we consider the 4-dimensional space of eigenfunctions with singularity at $q_{0}$. We could obtain a basis by adjoining any of the eigenfunctions constructed in section 6 , since these extend to the blow-up by spectral decimation using (6.5) to determine $c_{j}$ for negative values of $j$. But we would like to pick out one solution for which $c_{j} \rightarrow 0$ as $j \rightarrow-\infty$. This is clearly unique (up to a constant multiple) since all these functions are skew-symmetric, and $Q_{-\lambda}(x)$ blows up along $F_{0}^{-j} q_{1}$.

Since (6.5) tells us that

$$
\widetilde{c}_{j-1}=\left(\frac{2-\lambda_{j}}{6-\lambda_{j}}\right) \widetilde{c}_{j}
$$


and $\frac{2-\lambda_{j}}{6-\lambda_{j}} \rightarrow 1$ rapidly as $j \rightarrow-\infty$, we may conclude that $\widetilde{c}_{j}$ is bounded and tends to a limit as $j \rightarrow-\infty$. We may take the limit to be 1 . Then

$$
\widetilde{c}_{j}=\prod_{n=-\infty}^{j}\left(\frac{6-\lambda_{n}}{2-\lambda_{n}}\right) .
$$

Note that (7.9) is consistent with (6.7), and substituting (7.9) for $j=1$ into (6.8) gives a formula for $A$ in terms of the values $\left\{\lambda_{j}\right\}$. We also note that (6.5) may be written (using $\lambda_{j-1}=\lambda_{j}\left(5-\lambda_{j}\right)$ )

$$
\frac{c_{j}}{\lambda_{j}}=\frac{\widetilde{c}_{j}}{\lambda_{j-1}}+\frac{c_{j-1}}{\lambda_{j-1}} .
$$

If we want $\lim _{j \rightarrow-\infty} c_{j}=0$ we should take

$$
c_{j}=\lambda_{j} \sum_{k=1}^{\infty} \frac{\widetilde{c}_{-k+1}}{\lambda_{j-k}} .
$$

Indeed, the infinite series in (7.11) converges, because the numerators are bounded and denominators go to infinity rapidly. Then we can check that (7.10) holds, and

$$
\lim _{j \rightarrow-\infty} c_{j}=0
$$

because the ratios $\lambda_{j} / \lambda_{j-k}$ tend to zero rapidly. Although all the equations are explicit, it seems difficult to determine the values of $\alpha$ and $\beta$ in (6.14) for this particular eigenfunction.

\section{NONGENERIC EIGENFUNCTIONS ON BLOW-UPS}

Continuing the notation of section 7 , we consider what happens if $\lambda$ is nongeneric or $\lambda_{0} \in J$. In the nongeneric case, we have $\lambda_{m_{0}}=2,5$ or 6 for some $m_{0}$. Some of these eigenfunctions are described in [T1] because they contribute to the $L^{2}$ spectrum of the blow-up. Note that if $\lambda_{m_{0}}=6$, then $\lambda_{m_{0}+1}=2$ or 3 . For simplicity of notation, we will take $m_{0}=0$, since the general case just requires a rescaling.

Case 8.1. $\lambda_{0}=6$ and $\lambda_{1}=3$.

In this case, the space of eigenfunctions is infinite dimensional, and there is a one-to-one correspondence with the space of functions on $V_{-1}$. In other words, we may specify $u(x)$ for $x \in V_{-1}$ in any way. Then we extend $u$ to $V_{0}$ using the $\lambda=6$ spectral decimation formula,

$$
u(x)=\frac{1}{2}[u(a)-u(b)-u(c)] \quad \text { for } x \in V_{0} \backslash V_{-1},
$$

where $a$ is the opposite vertex and $b$ and $c$ are the closer vertices of the level -1 cell containing $x$. Of course, (8.1) guarantees that $u$ satisfies the 6-eigenfunction equation on level 0 for points in $V_{0} \backslash V_{-1}$. But it is also easy to verify that the same equation holds at points in $V_{-1}$. Indeed, suppose $a \in V_{-1}$ and $b, c$ and $b^{\prime}, c^{\prime}$ are the other vertices of the two level -1 cells containing $a$. Let $x, y$ and $x^{\prime}, y^{\prime}$ be the neighboring vertices in $V_{0} \backslash V_{-1}$. Then $u(x)+u(y)=\frac{1}{2}[u(b)-u(a)-u(c)]+$ $\frac{1}{2}[u(c)-u(a)-u(b)]=-u(a)$ by (8.1), and similarly $u\left(x^{\prime}\right)+u\left(y^{\prime}\right)=-u(a)$, and so $u(x)+u(y)+u\left(x^{\prime}\right)+u\left(y^{\prime}\right)=-2 u(a)$, which is the 6-eigenvalue equation at $a$.

We may then continue to extend $u$ to $V_{m}$ for all $m \geq 1$ by spectral decimation since we never again encounter 2, 5 or 6 for $\lambda_{m}$. When $\left.u\right|_{V_{-1}} \in l^{2}\left(V_{-1}\right)$, we obtain 


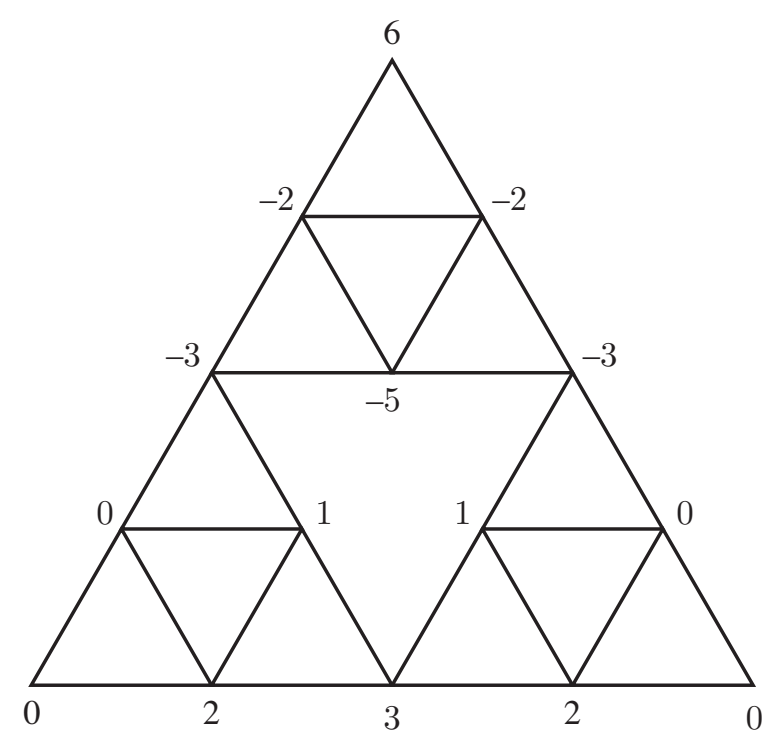

Figure 8.1. The extension to all points in $V_{1}$ from boundary values $(6,0,0)$ satisfying the 2-eigenvalue equation at all points except the boundary.

an $L^{2}$ eigenfunction as described in [T1] (using slightly different normalization conventions). If $\left.u\right|_{V_{-1}} \in l^{\infty}\left(V_{-1}\right)$, then we obtain a bounded eigenfunction.

Case 8.2. $\lambda_{0}=6$ and $\lambda_{1}=2$.

In this case, the eigenspace is 3-dimensional. As we will see, spectral decimation holds at all levels except level 0 , and a modified version goes from $V_{-1}$ to $V_{1}$.

Consider a cell of level -1 , and suppose the values of $u$ are given on the boundary. Then spectral decimation determines the values on the three points in $V_{0} \backslash V_{-1}$ in the cell, so that the 6-eigenvalue equation at level 0 holds at these three points. It turns out that there is a unique extension to the points in $V_{1} \backslash V_{0}$ so that the 2-eigenvalue equation on level 1 holds at all the points in $V_{1} \backslash V_{-1}$ in the cell. That extension is shown in Figure 8.1 for boundary values $(6,0,0)$. It is easy to check that the 2-eigenvalue equation is satisfied, and it may be checked that this is the unique extension with this property. Note that at a boundary point $a$ with neighbors $x, y$ on level 1 , the extension algorithm implies $u(x)+u(y)=\left[-\frac{1}{3} u(a)+\frac{1}{3} u(c)\right]+$ $\left[-\frac{1}{3} u(a)+\frac{1}{3} u(b)\right]$, where $b, c$ are the other level -1 vertices. The analogous result holds on the other level -1 cell containing $a$, so the 2-eigenvalue equation on level 1 at $a$ becomes $10 u(a)=u(b)+u(c)+u\left(b^{\prime}\right)+u\left(c^{\prime}\right)$, where $b, c, b^{\prime}, c^{\prime}$ are the neighboring points to $a$ on level -1 . But this is exactly the -6 -eigenvalue equation on level -1 at $a$, and $-6=\lambda_{-1}$. In other words, if $\left.u\right|_{V_{-1}}$ is a -6 -eigenfunction and we perform the extension exhibited in Figure 8.1 on every cell of level -1, then the extension is a 2-eigenfunction on level 1.

To actually construct a global eigenfunction we would start with the 2-eigenfunction on level 1 shown in Figure 8.1 on a cell of level -1 . We can then use outward spectral decimation to extend it inductively to a $\lambda_{n}$-eigenfunction on level $n$ on a cell $C_{n}$ of level $n$ for $n=-2,-3, \ldots$. Then we use inward spectral decimation to 


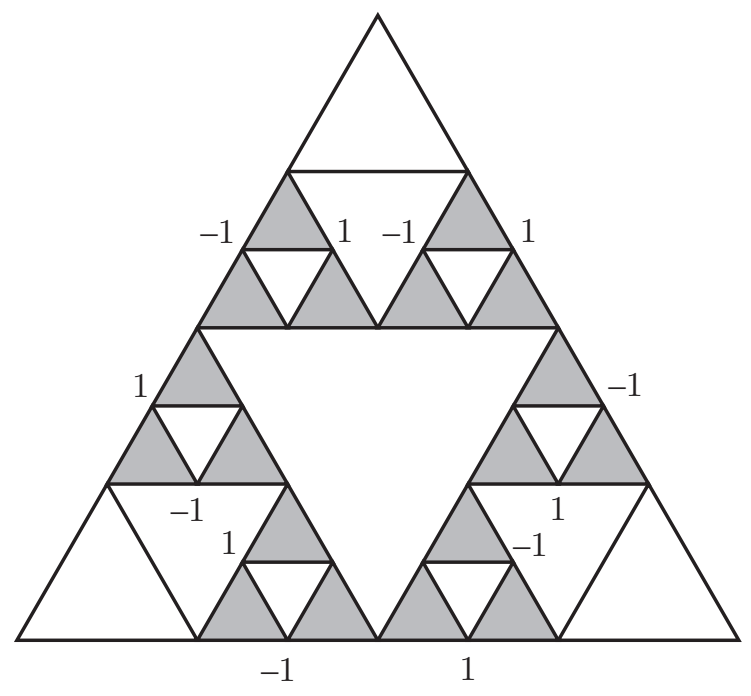

Figure 8.2. The 5-eigenfunction on level 0 on $V_{0}$ in a cell of level -3 . (Unmarked vertices have value 0 .)

extend it to a -6 -eigenfunction on level -1 on all the $V_{-1}$ vertices in $C_{n}$. Then we use the modified spectral decimation of Figure 8.1 to extend to a 2-eigenfunction on level 1 on all $V_{1}$ vertices in $C_{n}$. When we take the union over all negative $n$ we obtain a 2-eigenfunction on level 1 on all of $V_{1}$, and after that inward spectral decimation produces a $\lambda$-eigenfunction on $\mathrm{SG}_{w}$. If we do the same with the other two rotations of Figure 8.1, we obtain three linearly independent $\lambda$-eigenfunctions. We can then find special eigenfunctions using the methods of section 7 , just as in the non-Julia generic case.

Case 8.3. $\lambda_{0}=5$.

In this case, it is known (T1]) how to construct an eigenfunction supported in any cell $C_{n}$ of level $n \leq-2$. In fact, the support is the cycle of level -1 cells that goes around the inner triangular loop. The case $n=-3$ is illustrated in Figure 8.2. Each level -1 cell intersects the support of only 3 such functions, corresponding to the inner triangular loops containing the 3 sides of the triangle containing the cell. Thus any infinite linear combination of these functions defines a $\lambda$-eigenfunction. The function is $L^{2}$ if and only if the suitably normalized coefficients are in $l^{2}$, and is bounded if and only if the nonnormalized coefficients are bounded.

This does not quite exhaust the $\lambda$-eigenspace, however. In fact, the above is a subspace of codimension 2. To construct the missing eigenfunctions, we consider the doubly infinite chain of cells of level -1 , connecting $\mathcal{C}^{(0)}$ and $\mathcal{C}^{(1)}$. We easily construct a $\lambda$-eigenfunction supported in the chain by imitating the construction in Figure 8.2. We can do the same construction replacing the pair $\left(\mathcal{C}^{(0)}, \mathcal{C}^{(1)}\right)$ with $\left(\mathcal{C}^{(1)}, \mathcal{C}^{(2)}\right)$ to get the second missing eigenfunction. We do not get anything new using the pair $\left(\mathcal{C}^{(0)}, \mathcal{C}^{(2)}\right)$ because it is a linear combination of the previous two and the finite loop eigenfunctions. 
Case 8.4. $\lambda_{0}=4$.

In this case, the eigenvalue is generic, so there is a 3-dimensional space of eigenfunctions, but $\lambda_{0} \in J$. In fact, 4 is an expanding fixed point of the polynomial, so $\lambda_{n}=4$ for all $n \leq 0$. The spectral decimation formula for $\lambda_{0}=4$ is the same going outward and inward, namely $u(x)=-u(a)$ if $x \in V_{0} \backslash V_{-1}$ and $a \in V_{-1}$ is the vertex opposite $x$ in the level -1 cell containing $x$. Since the same is true on any level $n \leq 0$, it follows that all eigenfunctions are bounded, but none tend to zero along any path to infinity. In fact, the restriction to every cell of level 0 is identical to one of a finite number of functions. This is a kind of quasi-periodic behavior.

In the case of the blow-up with boundary point $q_{0}$, the eigenfunctions with singularity at $q_{0}$ may be described explicitly as in section 7 . In this case, (6.5) tells us that $\widetilde{c}_{j-1}=-\widetilde{c}_{j}$ for $j \leq 0$, so we may take

$$
\widetilde{c}_{j}=(-1)^{j} \quad \text { and } \quad c_{j}= \begin{cases}1 & j \text { even } \\ 0 & j \text { odd }\end{cases}
$$

for $j \leq 0$. In particular, the eigenfunction remains bounded away from the singularity, but does not decay at infinity.

Case 8.5. $\lambda_{0}=0$.

In this case, we are dealing with harmonic functions. In [S3], it was shown that there are no nonconstant harmonic functions that are bounded. But we can say even more: there are no nonconstant harmonic functions that are bounded on any of the chains $\mathcal{C}^{(i)}$. Indeed, consider the cell $F_{w_{1}}^{-1} F_{w_{2}}^{-1} \cdots F_{w_{m}}^{-1} K$ when $w_{m} \neq i$. The chain $\mathcal{C}^{(i)}$ must pass along half of one of the sides of the cell, and so contain the three points $F_{w_{1}}^{-1} F_{w_{2}}^{-1} \cdots F_{w_{m}}^{-1} q_{i}, F_{w_{1}}^{-1} F_{w_{2}}^{-1} \cdots F_{w_{m}}^{-1} F_{j} q_{i}$ and $F_{w_{1}}^{-1} F_{w_{2}}^{-1} \cdots F_{w_{m}}^{-1} F_{j} F_{j} q_{i}$ for some $j$. The values at these three points uniquely determine the harmonic function, in particular the values at the other two boundary points of the cell, via a linear identity whose coefficients are independent of $m$. In particular, if there were a uniform bound for the harmonic function on these three points in the cell, this would yield a uniform bound at the boundary of the cell, hence on the whole cell, and this would contradict the unboundedness of the nonconstant harmonic function.

Case 8.6. $\lambda_{0}=3 \pm \sqrt{3}$.

These are the period 2 points in $J$. Thus it is natural to look at spectral decimation two steps at a time. If we start with the values of $u$ on the boundary of a cell of level $-2 n$ and contract twice in the same direction, the boundary values (now on a cell of level $-2 n+2$ ) are multiplied by the matrix

$$
M_{1}=\left(\begin{array}{ccc}
1 & 0 & 0 \\
1 \pm \sqrt{3} & -1 & -2 \\
1 \pm \sqrt{3} & -2 & -1
\end{array}\right)
$$

or some permutation of it. Note that the eigenvalues of $M_{1}$ are 1 (with multiplicity 2) and -3 . Also, the intersection of the 1-eigenspaces for all 3 versions of $M_{1}$ is trivial. The inverse of $M_{1}$ has eigenvalues 1 and $-1 / 3$, and gives the boundary values on a cell of level $-2 n-2$ if the expansion is taken twice in the same direction. On the other hand, if we expand twice in different directions, then boundary values 
are multiplied by the matrix

$$
M_{2}^{-1}=\frac{1}{3}\left(\begin{array}{ccc}
\sqrt{3}+1 & 4-\sqrt{3} & 2 \sqrt{3}-2 \\
\sqrt{3} & 3-2 \sqrt{3} & \sqrt{3}-3 \\
-2 & 1-2 \sqrt{3} & \sqrt{3}-2
\end{array}\right)
$$

or some permutation of it. Note that $M_{2}^{-1}$ has eigenvalues $1, \frac{-1 \pm \sqrt{13}}{6}$, all of which exceed $1 / 3$ in absolute value.

Now we claim that if the blow-up has a boundary point, say $w=(0,0,0, \ldots)$, then there exists a bounded eigenfunction. Start with initial values

$$
\left(u\left(q_{0}\right), u\left(q_{1}\right), u\left(q_{2}\right)\right)=(0,1,1) .
$$

Note that this is the eigenvector of $M_{1}^{-1}$ with eigenvalue $-1 / 3$. Thus

$$
\left(u\left(q_{0}\right), u\left(F_{0}^{-2 n} q_{1}\right), u\left(F_{0}^{-2 n} q_{2}\right)\right)=\left(0,(-3)^{-n},(-3)^{-n}\right),
$$

so $u$ is very small $\left(O\left(3^{-n}\right)\right)$ on the boundary of $F_{0}^{-2 n} K$. To obtain the boundary values of $u$ on any cell of level 0 contained in $F_{0}^{-2 n} K$ we would multiply by $n$ matrices of the form $M_{1}$ or $M_{2}$ (or permutations). Since the largest eigenvalue of either of these (in absolute value) is 3 , we obtain a universal upper bound for $u$ on $V_{0}$, and this shows that $u$ is bounded. Incidentally, $u$ does not vanish at infinity in any direction, even though the values $u\left(F_{0}^{-2 n} q_{1}\right)$ and $u\left(F_{0}^{-2 n} q_{2}\right)$ do tend to zero. The bounded eigenfunction we have found corresponds to $S_{-\lambda}$ in the notation of part I.

Now suppose the blow-up has no boundary point. Then we claim that there are no bounded eigenfunctions. Again we start with any nonzero boundary values on $K$, and blow-up $2 n$ times. This will multiply by $n$ matrices of the form $M_{1}^{-1}$ and $M_{2}^{-1}$ (or permutations). A lower bound on the size we can obtain is

$$
3^{-n_{1}}\left(\frac{\sqrt{13}-1}{6}\right)^{-n_{2}},
$$

where $n_{1}+n_{2}=n$ and $n_{j}$ is the number of choices of $M_{j}^{-1}$ type matrix. We can then contract $n$ times in one of the 3 directions so that the values are multiplied by $3^{n}$. We end up with a cell of level 1 , where the boundary values are on the order of

$$
3^{n_{2}}\left(\frac{\sqrt{13}-1}{6}\right)^{n_{2}} \text {. }
$$

So if $n_{2} \rightarrow \infty$ as $n \rightarrow \infty$ we see that $u$ is unbounded. It may happen that $n_{2}$ remains bounded; for example, if $w=(0,0,1,1,0,0,1,1, \ldots)$, then $n_{2}=0$. In that case, we simply have to shift the cycle by one, starting with $F_{0}^{-1} K$ rather than $K$. Since $w_{j+1} \neq w_{j}$ infinitely often, one or the other case must work. Figure 8.3 shows an example of an unbounded eigenfunction for the case $w=(0,1,0,1, \ldots)$.

We believe that this case is typical of all examples with $\lambda_{0}$ in $J$ and $\lambda_{0} \neq 4$. Suppose that $\lambda_{0}$ is a periodic point in $J$ with period $p$. Consider the matrix analogous to $M_{1}$ above, where we contract $p$ times in the same direction. It is easy to see that there are three eigenvectors,

$$
\left(\begin{array}{l}
1 \\
a \\
a
\end{array}\right),\left(\begin{array}{l}
0 \\
1 \\
1
\end{array}\right) \text { and }\left(\begin{array}{c}
0 \\
1 \\
-1
\end{array}\right)
$$



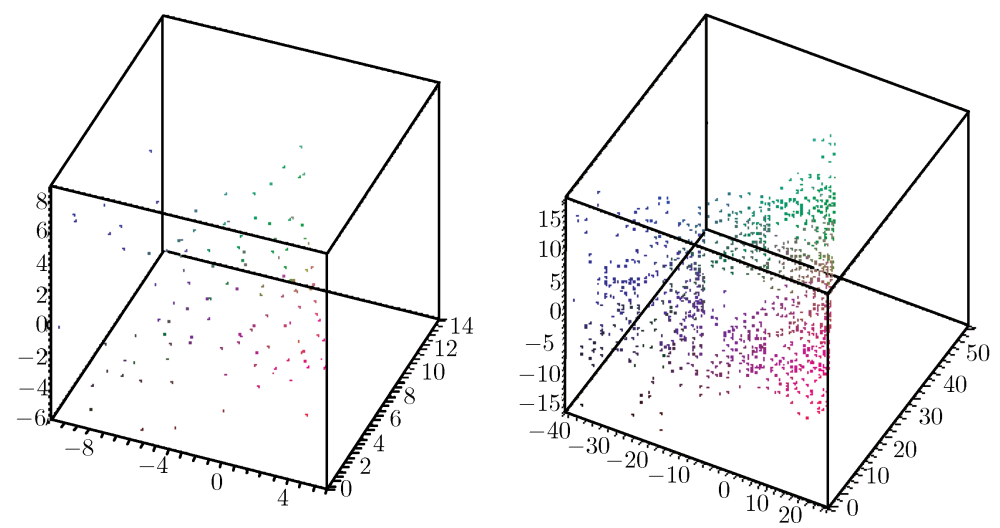

FiguRe 8.3. An eigenfunction corresponding to Case 8.6 with $\lambda_{0}=3+\sqrt{3}$ for the blow-up with $w=(0,1,0,1, \ldots)$, shown on two consecutive finite blow-ups. Note that the maximum value of the function increases as the domain expands, indicating that the function is unbounded.

(for the appropriate choice of $a$ ). The first has eigenvalue 1. The third has eigenvalue equal to the product of $\frac{1}{5-\lambda}$ over the cycle, which also equals 1 by (6.2). The eigenvalue of the second eigenvector is the product of $\frac{6-\lambda}{(2-\lambda)(5-\lambda)}$ over the cycle, which is the same as the product of $\frac{6-\lambda}{2-\lambda}$ over the cycle. If this number is greater than 1 in absolute value, then we can essentially repeat the argument given above, since we can estimate from below the eigenvalues of $M_{2}^{-1}$.

We tested this on the three periodic cycles of period $p=4$. The products were $-25.698,-2.72$ and 7.427 , corresponding to $\lambda_{0}=0.059188 \ldots, 1.031512 \ldots$ and $0.183262 \ldots$

\section{Normal DERIVATIVES}

Let $u$ be a Dirichlet eigenfunction, and fix a boundary point, say $q_{0}$. We wish to compute $\partial_{n} u\left(q_{0}\right)$. Since $u\left(q_{0}\right)=0$, the definition yields

$$
\partial_{n} u\left(q_{0}\right)=-\lim _{m \rightarrow \infty}\left(\frac{5}{3}\right)^{m}\left[u\left(F_{0}^{m} q_{1}\right)+u\left(F_{0}^{m} q_{2}\right)\right] .
$$

Suppose $m$ is large enough that spectral decimation is valid on level $m$. Then

$$
u\left(F_{0}^{m} q_{1}\right)+u\left(F_{0}^{m} q_{2}\right)=\frac{\left(6-\lambda_{m}\right)}{\left(2-\lambda_{m}\right)\left(5-\lambda_{m}\right)}\left[u\left(F_{0}^{m-1} q_{1}\right)+u\left(F_{0}^{m-1} q_{2}\right)\right] .
$$

This leads to the infinite product formula

$$
\partial_{n} u\left(q_{0}\right)=A_{m_{0}} \prod_{m=m_{0}+1}^{\infty} \frac{\left(1-\frac{1}{6} \lambda_{m}\right)}{\left(1-\frac{1}{2} \lambda_{m}\right)\left(1-\frac{1}{5} \lambda_{m}\right)}
$$

for

$$
A_{m_{0}}=-\left(\frac{5}{3}\right)^{m_{0}}\left[u\left(F_{0}^{m_{0}} q_{1}\right)+u\left(F_{0}^{m_{0}} q_{2}\right)\right]
$$


Now suppose $u$ is normalized $\left(\|u\|_{2}=1\right)$. Then we can use Corollary 2.3 of OSS to control the size of $u$ on $V_{m_{0}}$. Specifically, if $m_{0}=1$, then

$$
1=2 \cdot 3^{-2} \prod_{m=2}^{\infty} \frac{\left(1-\frac{1}{6} \lambda_{m}\right)\left(1-\frac{2}{5} \lambda_{m}\right)}{\left(1-\frac{1}{5} \lambda_{m}\right)\left(1-\frac{1}{2} \lambda_{m}\right)}\left[u\left(F_{0} q_{1}\right)^{2}+u\left(F_{0} q_{2}\right)^{2}+u\left(F_{1} q_{2}\right)^{2}\right] .
$$

If $\lambda_{1}=2$, then $u\left(F_{0} q_{1}\right)=u\left(F_{0} q_{2}\right)=u\left(F_{1} q_{2}\right)$. Suppose we choose them to be negative. Then

$$
\partial_{n} u\left(q_{0}\right)=\frac{10}{3} \sqrt{\frac{3}{2}}\left[\prod_{m=2}^{\infty} \frac{\left(1-\frac{1}{6} \lambda_{m}\right)}{\left(1-\frac{1}{2} \lambda_{m}\right)\left(1-\frac{1}{5} \lambda_{m}\right)\left(1-\frac{2}{5} \lambda_{m}\right)}\right]^{1 / 2} .
$$

We can simplify this somewhat since

$$
\prod_{m=2}^{\infty} \frac{1}{\left(1-\frac{1}{5} \lambda_{m}\right)}=\prod_{m=2}^{\infty} \frac{5^{m} \lambda_{m}}{5^{m-1} \lambda_{m-1}}=\frac{2}{15} \frac{\lambda}{\lambda_{1}} .
$$

If we define a function $c(t)$ by

$$
c(t)=\frac{\left(1-\frac{1}{6} t\right)}{\left(1-\frac{1}{2} t\right)\left(1-\frac{2}{5} t\right)}
$$

then

$$
\partial_{n} u\left(q_{0}\right)=\frac{\sqrt{10}}{3} \lambda^{1 / 2}\left[\prod_{m=2}^{\infty} c\left(\lambda_{m}\right)\right]^{1 / 2} .
$$

Similarly, if $\lambda_{1}=5$, then there is a 2-dimensional space of eigenfunctions. If the eigenfunction is skew-symmetric with respect to $R_{0}$, then $\partial_{n} u\left(q_{0}\right)=0$. For a symmetric eigenfunction, we will have $u\left(F_{0} q_{1}\right)=u\left(F_{0} q_{2}\right)=-\frac{1}{2} u\left(F_{1} q_{2}\right)$. Taking $u\left(F_{0} q_{1}\right)$ to be negative, we have

$$
\partial_{n} u\left(q_{0}\right)=\frac{\sqrt{2}}{3} \lambda^{1 / 2}\left[\prod_{m=2}^{\infty} c\left(\lambda_{m}\right)\right]^{1 / 2} .
$$

In order to estimate the normal derivative, we need to bound the infinite product in (9.9) and (9.10). Each sequence $\left\{\lambda_{m}\right\}$ is determined from $\lambda_{1}=2$ or 5 and

$$
\lambda_{m+1}=\frac{1}{2}\left(5+\varepsilon_{m} \sqrt{25-4 \lambda_{m}}\right), \quad \varepsilon_{m}= \pm 1 .
$$

We want to show that the product is largest when all $\varepsilon_{m}=-1$. Let

$$
\widetilde{c}(t)=\prod_{j=1}^{\infty} c\left(t_{j}\right)
$$

for $t_{1}=t$ and

$$
t_{m+1}=\frac{1}{2}\left(5-\sqrt{25-4 t_{m}}\right) .
$$

If all $\varepsilon_{m}=-1$, then

$$
\prod_{m=2}^{\infty} c\left(\lambda_{m}\right)=\left\{\begin{array}{l}
\widetilde{c}\left(\frac{5-\sqrt{17}}{2}\right) \text { if } \lambda_{1}=2 \\
\widetilde{c}\left(\frac{5-\sqrt{5}}{2}\right) \text { if } \lambda_{1}=5
\end{array}\right.
$$




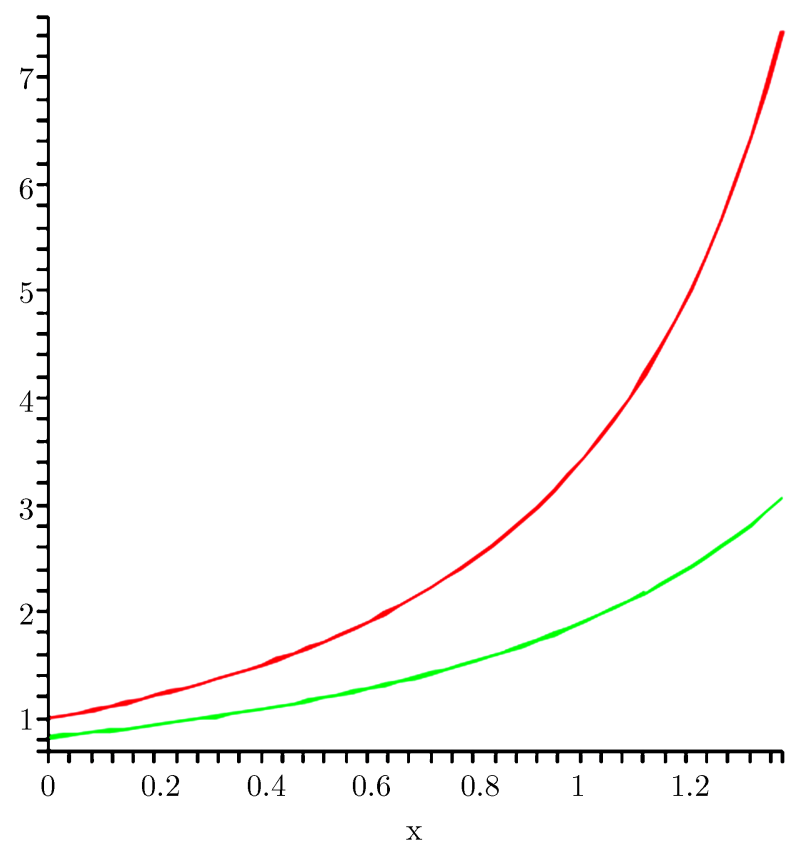

Figure 9.1. The graphs of $\widetilde{c}(x)$ (top) and $\widetilde{c}(5-x)$ (bottom) on the interval $\left[0, \frac{1}{2}(5-\sqrt{5})\right]$.

On the other hand, if $\varepsilon_{k}=+1$ and $\varepsilon_{m}=-1$ for all $m>k$, then

$$
\prod_{m=2}^{\infty} c\left(\lambda_{m}\right)=\left[\prod_{m=2}^{k} c\left(\lambda_{m}\right)\right] \widetilde{c}\left(\lambda_{k+1}\right) .
$$

If we change $\varepsilon_{k}$ to -1 , then the only change in $(9.15)$ is in $\widetilde{c}\left(\lambda_{k+1}\right)$.

Lemma 9.1. For any $t$ in $[0,5]$

$$
\widetilde{c}\left(\frac{1}{2}(5+\sqrt{25-4 t})\right)<\widetilde{c}\left(\frac{1}{2}(5-\sqrt{25-4 t})\right) .
$$

Proof. Note that (9.16) is equivalent to

$$
\widetilde{c}(5-x)<\widetilde{c}(x) \text { for } 0 \leq x \leq \frac{1}{2}(5-\sqrt{5}) .
$$

This is evident from the graphs in Figure 9.1.

Lemma 9.2. If $u$ is a normalized eigenfunction with $\lambda_{1}=2$ or 5 , then

$$
\left|\partial_{n} u\left(q_{0}\right)\right| \leq M \lambda^{1 / 2} .
$$

Proof. By (9.16), we increase (9.15) by changing $\varepsilon_{k}$ to -1 . Repeating the same argument, we can one by one change all $\varepsilon_{m}$ to -1 , increasing (9.15). Thus

$$
\prod_{m=2}^{\infty} c\left(\lambda_{m}\right) \leq\left\{\begin{array}{l}
\widetilde{c}\left(\frac{5-\sqrt{17}}{2}\right) \text { if } \lambda_{1}=2 \\
\widetilde{c}\left(\frac{5-\sqrt{5}}{2}\right) \text { if } \lambda_{1}=5
\end{array}\right.
$$

and we obtain (9.18) from (9.9) and (9.10). 
Note that the estimate (9.18) cannot be improved. If we take $\varepsilon_{k}=+1$ and all other $\varepsilon_{m}=-1$, then we easily obtain a lower bound for $\prod_{m=2}^{\infty} c\left(\lambda_{m}\right)$ of $c\left(\lambda_{k+1}\right)$, since all other $c\left(\lambda_{m}\right)$ are greater than 1 , and $c\left(\lambda_{k+1}\right)>c(5)=\frac{1}{9}$. On the other hand, for most eigenfunctions (9.18) is far from sharp. At the other extreme, suppose $\varepsilon_{m}=+1$ for $m \leq k$ and $\varepsilon_{m}=-1$ for $m>k$. Then $\lambda_{m} \approx 4$ for $m \leq k+1$ and $c(4)=\frac{5}{9}$. More precisely, if $\lambda_{m}=4+\delta_{m}$, then $\delta_{m+1} \approx-\frac{1}{3} \delta_{m}$, and $c\left(4+\delta_{m}\right) \approx$ $\frac{5}{9}-\frac{5}{27} \delta_{m}$. So $\prod_{m=2}^{\infty} c\left(\lambda_{m}\right)$ is bounded above and below by multiples of $\left(\frac{5}{9}\right)^{k}$. Thus $\left|\partial_{n} u\left(q_{0}\right)\right| \approx \lambda^{1 / 2}\left(\frac{5}{9}\right)^{k / 2}$. Since $\lambda \approx 5^{k}$, we can write this as

$$
\left|\partial_{n} u\left(q_{0}\right)\right| \approx \lambda^{\log \frac{5}{3} / \log 5}
$$

along this sequence of eigenvalues. Note that $\log \frac{5}{3} / \log 5=1 /(d+1) \approx 0.3173938$. It is not hard to show that (9.20) is always a lower bound.

Next we consider other Dirichlet eigenfunctions. Of course, for joint Dirichlet and Neumann eigenfunctions, $\partial_{n} u\left(q_{0}\right)=0$. In particular, this covers all eigenfunctions when $\lambda_{k}=6$ and $\lambda_{k+1}=3$ for some $k \geq 2$. When $\lambda_{k}=5$ for some $k \geq 2$, the joint Dirichlet and Neumann eigenfunctions have codimension 2 in the space of Dirichlet eigenfunctions. Thus we can choose an orthonormal basis for the Dirichlet eigenspace so that $\partial_{n} u\left(q_{0}\right)=0$ for all except 2 basis elements. If we choose a symmetric and skew-symmetric (with respect to $R_{0}$ ) eigenfunction from the 2dimensional space, then the skew-symmetric one must have $\partial_{n} u\left(q_{0}\right)=0$. Thus it remains to compute $\partial_{n} u\left(q_{0}\right)$ when $u$ is symmetric, normalized, and orthogonal to all joint Dirichlet and Neumann eigenfunctions. It turns out that we can do this computation exactly, and in fact give an inductive description of this eigenfunction.

For this discussion we let $u_{1}$ denote a symmetric $\lambda$-eigenfunction with $\lambda_{1}=5$. For the moment, we will not insist that $u_{1}$ be normalized, and the other eigenfunctions $u_{2}, u_{3}, \ldots$ that we construct will not be normalized. The idea is that $u_{k}$ will be a $\left(5^{k-1} \lambda\right)$-eigenfunction with $\lambda_{k}=5$ and

$$
\left\{\begin{array}{l}
\left.u_{k}\right|_{F_{0} K}=u_{k-1} \circ F_{0}^{-1}, \\
\left.u_{k}\right|_{F_{1} K}=\frac{1}{3}\left(u_{k-1}-u_{k-1} \circ R_{2}\right) \circ F_{1}^{-1}, \\
\left.u_{k}\right|_{F_{2} K}=\frac{1}{3}\left(u_{k-1}-u_{k-1} \circ R_{1}\right) \circ F_{2}^{-1} .
\end{array}\right.
$$

It is easy to check that $u_{k}$ is $R_{0}$ symmetric. To check that $u_{k}$ is actually an eigenfunction, we need to check the matching conditions on the normal derivatives at $F_{0} q_{1}, F_{0} q_{2}$ and $F_{1} q_{2}$. At $F_{1} q_{2}$ both $\left.u_{k}\right|_{F_{1} K}$ and $\left.u_{k}\right|_{F_{2} K}$ are skew-symmetric, and so have normal derivative equal to zero. At the other points, we begin with the observation $\partial_{n} u_{1}\left(q_{1}\right)=\partial_{n} u_{1}\left(q_{2}\right)=-\frac{1}{2} \partial_{n} u_{1}\left(q_{0}\right)$. We then observe from (9.21) that the matching conditions at $F_{0} q_{1}$ and $F_{0} q_{2}$ and

$$
\partial_{n} u_{k}\left(q_{1}\right)=\partial_{n} u_{k}\left(q_{2}\right)=-\frac{1}{2} \partial_{n} u_{k}\left(q_{0}\right)
$$

follow by induction, using the identity $\frac{1}{3}\left[1-\left(-\frac{1}{2}\right)\right]=\frac{1}{2}$. (This explains the choice of the constant $\frac{1}{3}$ in $(9.21)$.)

Lemma 9.3. The eigenfunction $u_{k}$ is orthogonal to all joint Dirichlet and Neumann eigenfunctions with the same eigenvalue.

Proof. When $k=2$, there is only one joint eigenfunction, looping around the inner triangle, and it is skew-symmetric. Since $u_{2}$ is symmetric, they are orthogonal. 


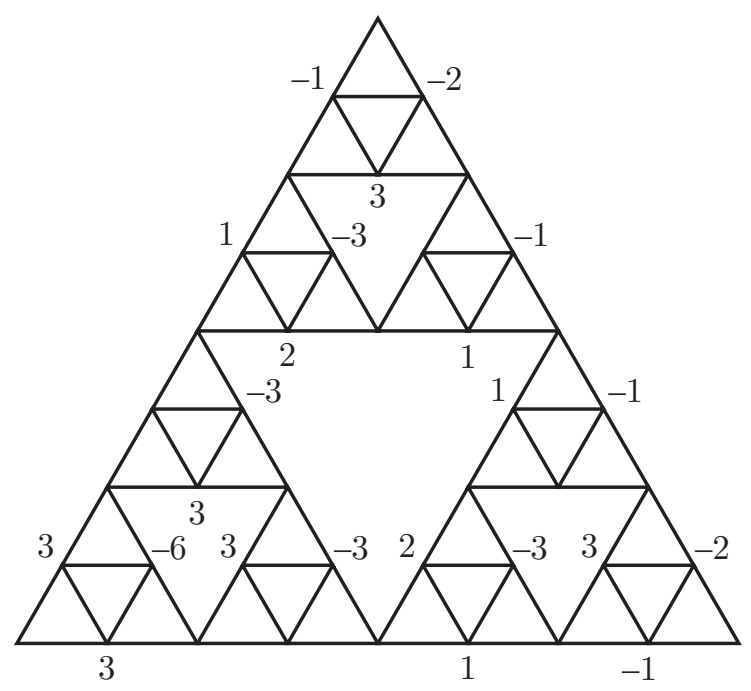

Figure 9.2. The values of $u_{3}$ on $V_{3}$ (normalized so all values are integers). By restricting to lower left triangles, one can read off the values of $u_{2}$ on $V_{2}$ and $u_{1}$ on $V_{1}$.

We prove the general case by induction, assuming that it is true for $k-1$. The above argument still shows that $u_{k}$ is orthogonal to the joint eigenfunction looping around the inner triangle. All the other basis elements for the joint eigenspace are supported in either $F_{0} K, F_{1} K$, or $F_{2} K$. Then we can use the induction hypothesis, since the orthogonality condition is preserved by reflection.

Having constructed the desired eigenfunctions, we now tackle the normalization problem. Figure 9.2 shows $u_{3}$ on $V_{3}$.

Lemma 9.4. For all $k \geq 1$, we have

$$
\int\left(u_{k} \circ R_{j}\right) u_{k} d \mu=-\frac{1}{2} \int\left|u_{k}\right|^{2} d \mu \quad \text { for } j=1,2,
$$

and

$$
\int\left|u_{k}\right|^{2} d \mu=\left(\frac{5}{9}\right)^{k-1} \int\left|u_{1}\right|^{2} d \mu .
$$

Proof. We will use Corollary 2.3 of [OSS], which tells us that

$$
\int\left|u_{k}\right|^{2} d \mu=\frac{a(\lambda)}{3^{k}} \sum_{x \in V_{k}}\left|u_{k}(x)\right|^{2}
$$

for a fixed constant $a(\lambda)$. We prove both identities by induction on $k$. When $k=1$, (9.24) is obvious, and we see that (9.23) holds because

$$
\sum_{x \in V_{1}} u_{1}(x) u_{1}\left(R_{j} x\right)=-\frac{1}{2} \sum_{x \in V_{1}} u_{1}(x)^{2} .
$$


Now assume both identities hold for $u_{k-1}$. Using (9.21), we have

$$
\begin{aligned}
\sum_{x \in V_{k}}\left|u_{k}(x)\right|^{2} & =\sum_{x \in V_{k-1}}\left|u_{k-1}(x)\right|^{2}+\frac{2}{9} \sum_{x \in V_{k-1}}\left|u_{k-1}(x)-u_{k-1}\left(R_{j} x\right)\right|^{2} \\
& =\frac{13}{9} \sum_{x \in V_{k-1}}\left|u_{k-1}(x)\right|^{2}-\frac{4}{9} \sum_{x \in V_{k-1}} u_{k-1}(x) u_{k-1}\left(R_{j} x\right) \\
& =\frac{5}{3} \sum_{x \in V_{k-1}}\left|u_{k-1}(x)\right|^{2} .
\end{aligned}
$$

Using (9.25) and the induction hypothesis yields (9.24). Similarly

$$
\begin{aligned}
\sum_{x \in V_{k}} u_{k}(x) u_{k}\left(R_{j} x\right)= & \sum_{x \in V_{k-1}} u_{k-1}(x) u_{k-1}\left(R_{j} x\right) \\
& +\frac{2}{9} \sum_{x \in V_{k-1}}\left[u(x)-u\left(R_{2} x\right)\right]\left[u\left(R_{2} x\right)-u\left(R_{1} x\right)\right] \\
= & -\frac{1}{2} \frac{5}{3} \sum_{x \in V_{k-1}}\left|u_{k-1}(x)\right|^{2},
\end{aligned}
$$

which proves (9.23).

Theorem 9.5. The ratio $\frac{\partial_{n} u\left(q_{0}\right)}{\lambda^{1 / 2}\|u\|_{2}}$ is the same for all $u_{k}$ constructed above. In particular, the estimate (9.18) holds for all normalized eigenfunctions.

Proof. Clearly, $\partial_{n} u_{k}\left(q_{0}\right)=\left(\frac{5}{3}\right)^{k-1} \partial_{n} u_{1}\left(q_{0}\right)$ and the eigenvalue of $u_{k}$ is $5^{k-1}$ times the eigenvalue of $u_{1}$, so (9.24) implies the ratio is constant.

Figure 9.3 shows a plot of the ratio $\partial_{n} u\left(q_{0}\right) / \sqrt{\lambda}$ on a log-log scale as a function of $\lambda$ for all eigenfunctions with $\partial_{n} u\left(q_{0}\right) \neq 0$, where the eigenvalues have $\varepsilon_{m}=-1$ for $m \geq 10$ (so there are 512 eigenvalues with $\lambda_{1}=2$ or 5 , etc.). It is clear from this figure that there is a rich self-similar type structure to the values $\partial_{n} u\left(q_{0}\right)$. In Figures 9.4, 9.5, and 9.6, we zoom in on the top half, quarter, and eighth of the data points. In Figure 9.7, we zoom in on the second quarter from the top, and in Figure 9.8 we display a mirror image of the same data. The similarities among Figures 9.4, 9.5, 9.6 and 9.8 are striking. At the present time, we have no explanation for this.

Next, we consider normal derivatives of the Dirichlet heat kernel. The heat kernel may be written as

$$
p_{t}(x, y)=\sum e^{-t \lambda_{j}} u_{j}(x) u_{j}(y)
$$

where $\left\{u_{j}\right\}$ denotes an orthonormal basis of Dirichlet eigenfunctions with associated eigenvalues $\left\{\lambda_{j}\right\}$. (Note this is different from our previous notation.) For any fixed value of $t>0$, we may obtain a good approximation by terminating the sum once $\lambda_{j} t$ becomes large. Looked at another way, if we take a partial sum up to $\lambda_{j} \leq N$, then we will have a reasonable approximation for values of $t$ such that $t N$ is reasonably large.

The normal derivatives of $p_{t}(x, y)$ that we are interested in may be written as

$$
\partial_{n} \partial_{n} p_{t}\left(q_{0}, q_{0}\right)=\sum e^{-\lambda_{j} t} \partial_{n} u_{j}\left(q_{0}\right)^{2} .
$$




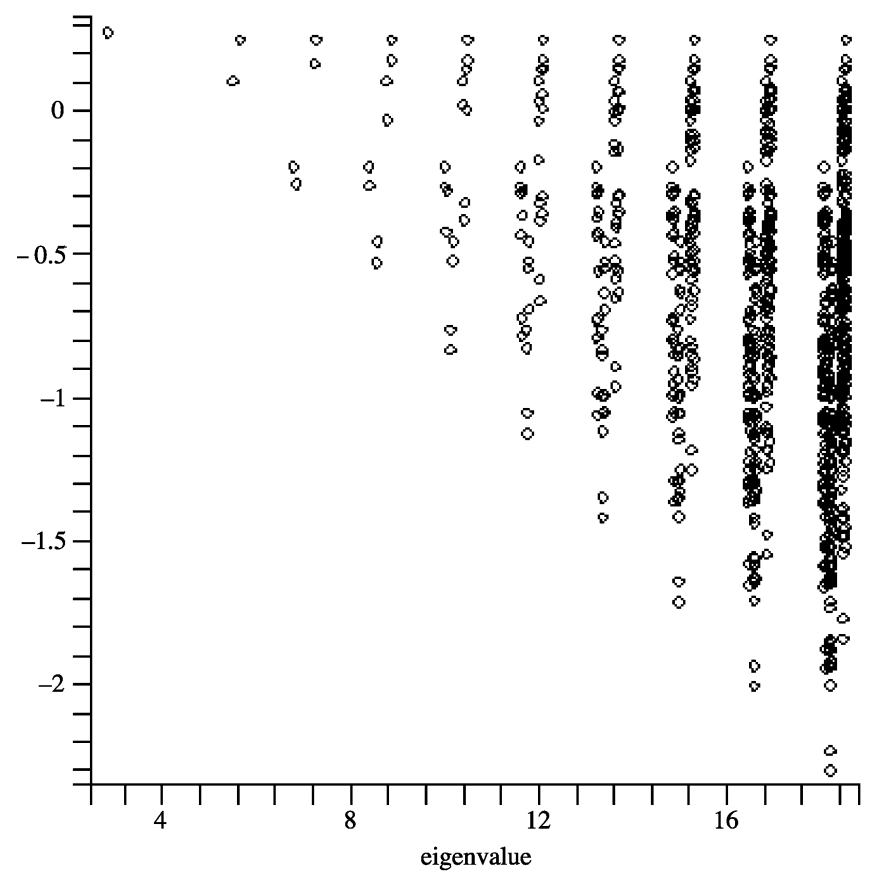

Figure 9.3. A log-log plot of the ratio $\partial_{n} u\left(q_{0}\right) / \sqrt{\lambda}$ as a function of $\lambda$.

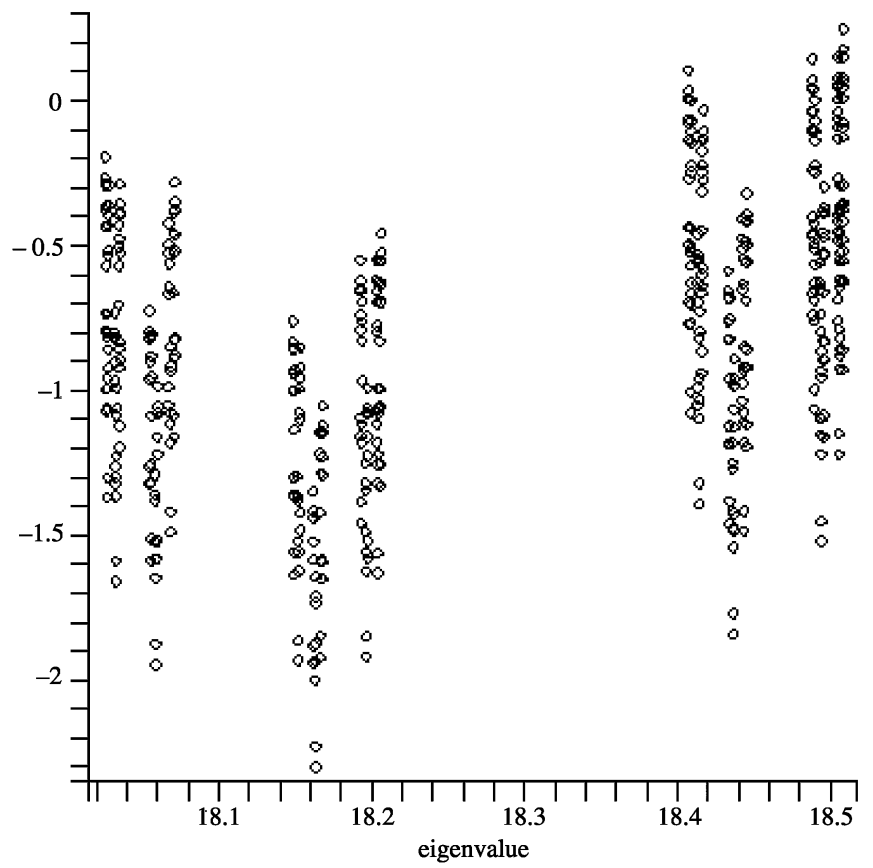

Figure 9.4. A zoom of the right-most vertical strip in Figure 9.3, representing the top half of the data points. 


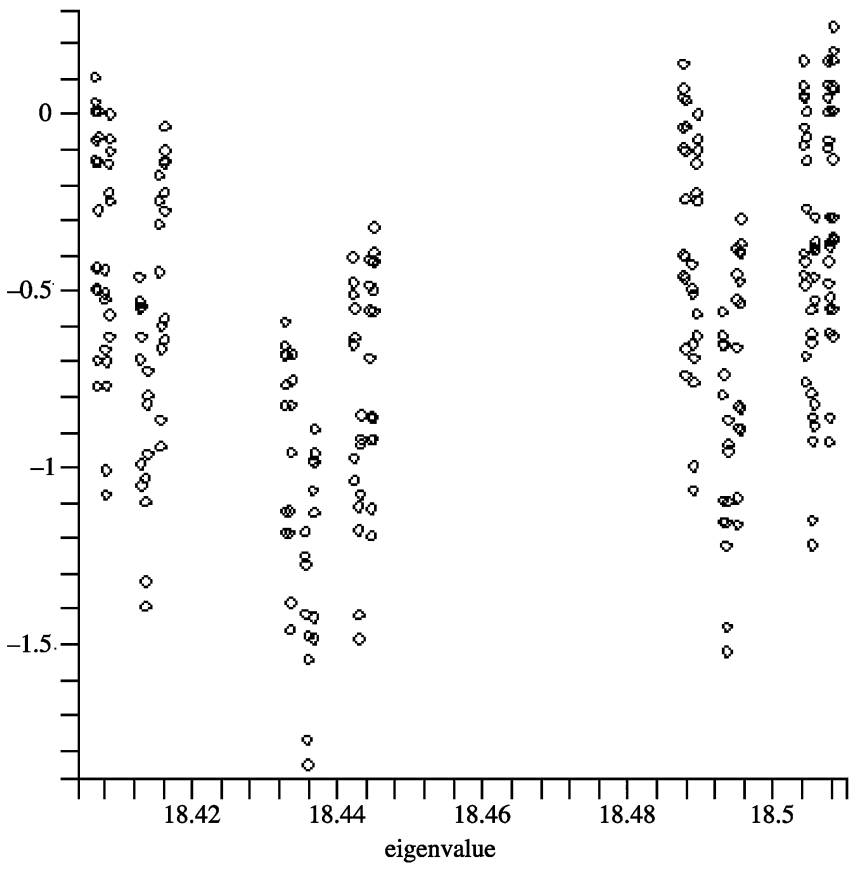

Figure 9.5. A zoom of the right half of Figure 9.4, representing the top quarter of data points in Figure 9.3.

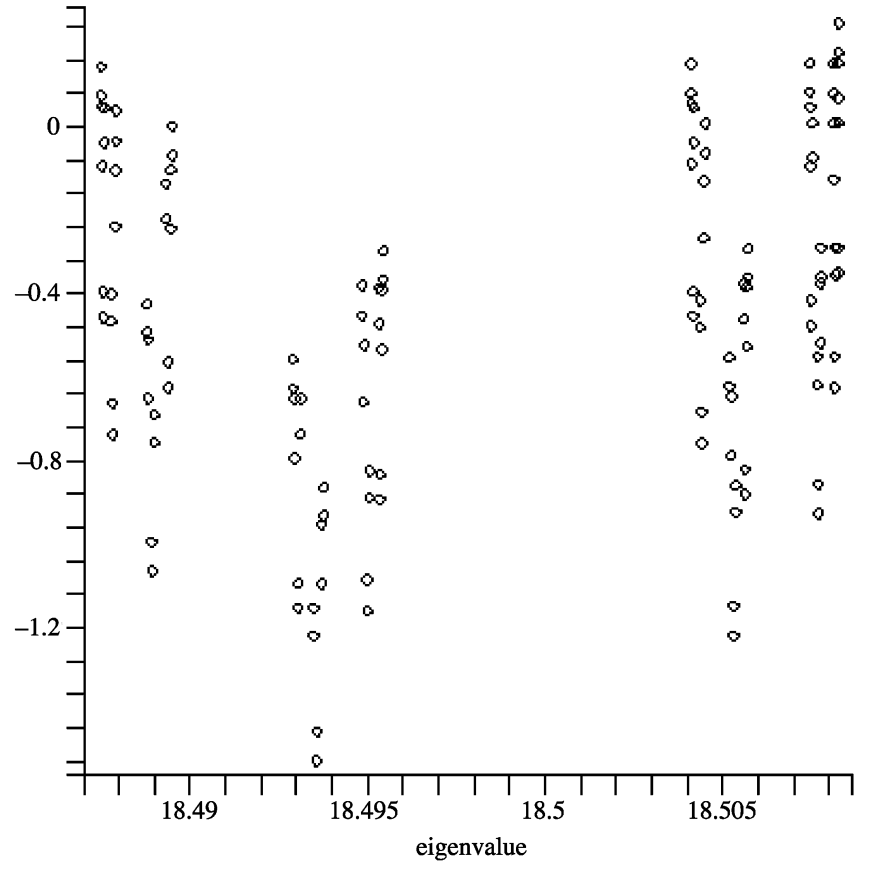

Figure 9.6. A zoom of the right half of Figure 9.5, representing the top eighth of data points in Figure 9.3. 


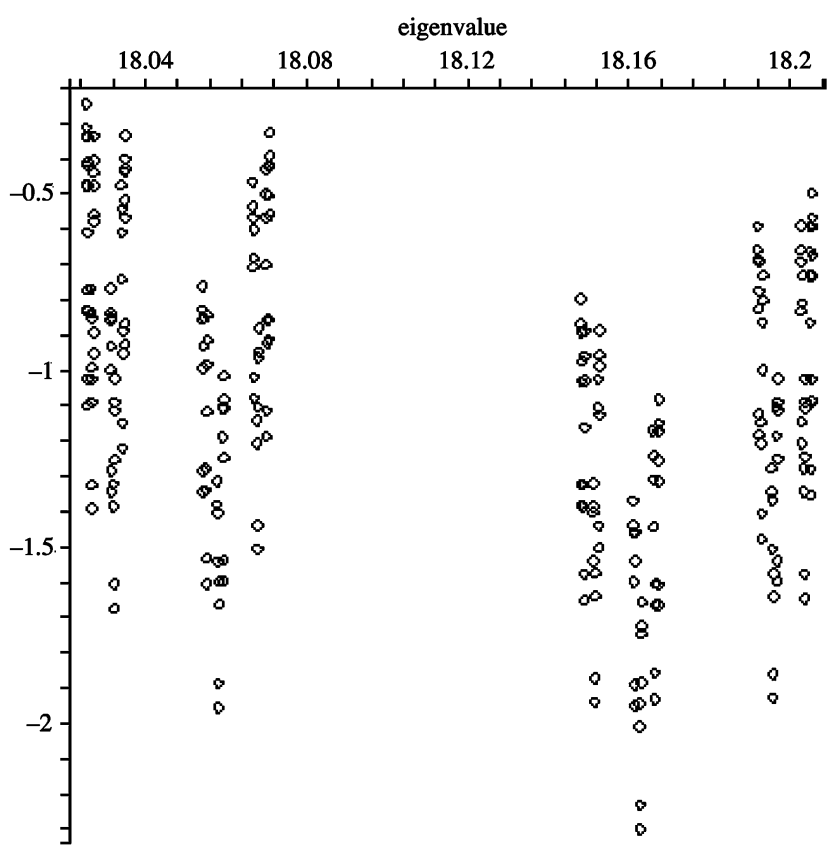

Figure 9.7. A zoom of the left half of Figure 9.4, representing the third quarter of data points in Figure 9.3.

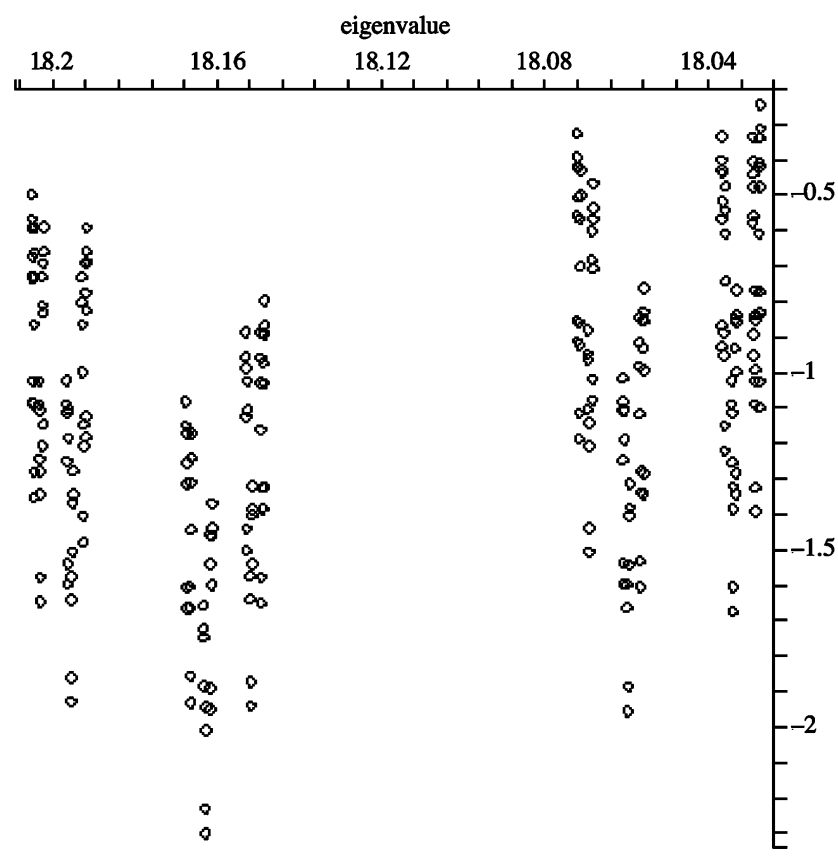

Figure 9.8. The same data points as in Figure 9.7, but with the order of the horizontal axis reversed. 


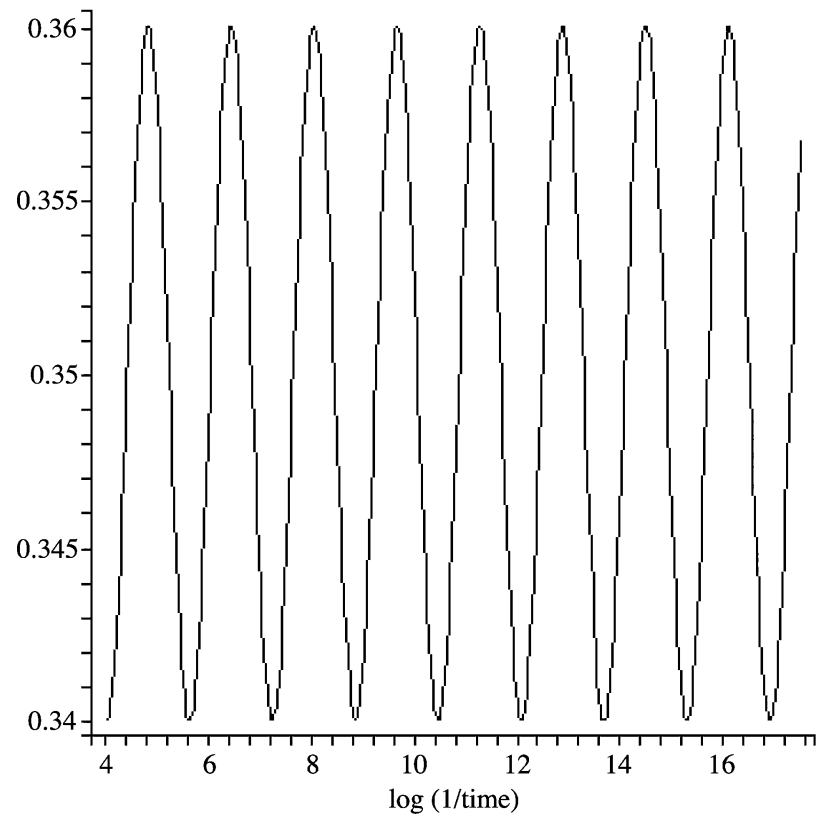

Figure 9.9. The graph of (9.30) as a function of $\log (1 / t)$.

(In other words, we take normal derivatives with respect to both variables at the same boundary point $q_{0}$.) Estimates for the heat kernel are known ( $\overline{\mathrm{Ba}}$, [Ki2]$)$; in particular, we have sharp upper bounds on the diagonal

$$
p_{t}(x, x) \leq c t^{-\log 5 / \log 3} \text { for } 0<t \leq 1,
$$

but the behavior as $x$ approaches the boundary has not been understood. In [S6], it was conjectured that

$$
\partial_{n} \partial_{n} p_{t}\left(q_{0}, q_{0}\right) \leq c t^{-\log \frac{25}{3} / \log 5} \text { for } 0<t \leq 1 .
$$

This estimate has important consequences for analysis on the product $\mathrm{SG} \times \mathrm{SG}$.

We are now in a position to present strong evidence that (9.29) holds and is sharp, in the sense that similar lower bounds hold. We approximate (9.27) by taking all the values of $\partial_{n} u_{j}\left(q_{0}\right)$ and $\lambda_{j}$ included in the data in Figure 9.3. In Figure 9.9, we show the graph of

$$
t^{\log \frac{25}{3} / \log 5} \partial_{n} \partial_{n} p_{t}\left(q_{0}, q_{0}\right)
$$

using this approximation, as a function of $\log (1 / t)$, so the behavior as $t \rightarrow 0$ is transformed to $\log (1 / t) \rightarrow \infty$. In the range $6 \leq \log (1 / t) \leq 16$ the function (9.30) exhibits a striking periodicity, with period $\log 5$.

Conjecture 9.6. (a) There exists a positive, continuous, periodic (of period $\log 5$ ) function $g$ such that

$$
t^{\log \frac{25}{3} / \log 5} \partial_{n} \partial_{n} p_{t}\left(q_{0}, q_{0}\right)-g(\log (1 / t)) \rightarrow 0
$$

as $t \rightarrow 0$. 
(b) For every nonboundary point $x \in K$, there exists a positive, continuous, periodic (of period $\log 5$ ) function $g_{x}$ such that

$$
t^{\log 5 / \log 3} p_{t}(x, x)-g_{x}(\log (1 / t)) \rightarrow 0
$$

as $t \rightarrow 0$.

There is ample precedent for asymptotic periodicity, as for example in the Weyl ratio for the spectrum (although the periodic function in that case is discontinuous). See [FS], KL], ASST]. Conjecture 9.6 (b) will be proven in AS.

\section{ACKNOWLEDGMENT}

We are grateful to Pavel Gyrya for helpful discussions.

\section{REFERENCES}

[ASST] B. Adams, S. A. Smith, R. Strichartz and A. Teplyaev, The spectrum of the Laplacian on the pentagasket, Trends in Mathematics: Fractals in Graz 2001, Birkhauser, 2003, pp. 1-24. MR2091699

[AS] A. Allan and R. Strichartz, Spectral operators on the Sierpinski gasket, in preparation.

[A-LS] C. Avenancio-Leon and R. Strichartz, Local behavior of harmonic functions on the Sierpinski gasket, preprint.

[Ba] M. Barlow, Diffusion on fractals, Lecture Notes Math., vol. 1690, Springer, 1998. MR.1668115 (2000a:60148)

[BST] O. Ben-Bassat, R. Strichartz and A. Teplyaev, What is not in the domain of the Laplacian on Sierpinski gasket type fractals, J. of Functional Analysis 166 (1999), 197-217. MR 1707752 (2001e:31016)

[DSV] K. Dalrymple, R. Strichartz and J. Vinson, Fractal differential equations on the Sierpinski gasket, J. Fourier Anal. Appl. 5 (1999), 203-284. MR1683211 (2000k:31016)

[FS] M. Fukushima and T. Shima, On a spectral analysis for the Sierpinski gasket, Potential Anal. 1 (1992), 1-35. MR.1245223 (95b:31009)

[GRS] M. Gibbons, A. Raj and R. Strichartz, The finite element method on the Sierpinski gasket, Constructive Approx. 17 (2001), 561-588. MR.1845268 (2002i:28010)

[Ki1] J. Kigami, A harmonic calculus on the Sierpinski spaces, Japan J. Appl. Math. 8 (1989), 259-290. MR1001286 (91g:31005)

[Ki2] J. Kigami, Analysis on Fractals, Cambridge University Press, New York, 2001. MR1840042 (2002c:28015)

[KL] J. Kigami and M. L. Lapidus, Weyl's problem for the spectral distribution of Laplacians on p.c.f. self-similar fractals, Comm. Math. Phys. 158 (1993), 93-135. MR.1243717 (94m:58225)

[KSS] J. Kigami, D. Sheldon and R. Strichartz, Green's functions on fractals, Fractals 8 (2000), 385-402. MR1810881 (2002f:31021)

[NSTY] J. Needleman, R. Strichartz, A. Teplyaev and P.-L. Yang, Calculus on the Sierpinski gasket I: polynomials, exponentials and power series, J. Functional Anal. 215 (2004), 290-340. MR2150975

[OSS] R. Oberlin, B. Street and R. Strichartz, Sampling on the Sierpinski gasket, Experimental Mathematics 12 (2003), 403-418. MR2043991(2005b:28018)

[OSY] A. Öberg, R. Strichartz and A. Yingst, Level sets of harmonic functions on the Sierpinski gasket, Ark. Mat. 40 (2002), 335-362. MR1948069 (2005b:28017)

[S1] R. Strichartz, Fractals in the large, Can. J. Math. 50 (1998), 638-657. MR.1629847 (99f:28015)

[S2] R. Strichartz, Analysis on fractals, Notices American Mathematical Society 46 (1999), 1199-1208. MR 1715511 (2000i:58035)

[S3] R. Strichartz, Some properties of Laplacians on fractals, J. Functional Anal. 164 (1999), 181-208. MR1695571 (2000f:35032)

[S4] R. Strichartz, Taylor approximations on Sierpinski gasket-type fractals, J. Functional Anal. 174 (2000), 76-127. MR.1761364 (2001i:31018) 
[S5] R. Strichartz, Fractafolds based on the Sierpinski gasket and their spectra, Trans. Amer. Math. Soc. 355 (2003), 4019-4043. MR1990573 (2004b:28013)

[S6] R. Strichartz, Analysis on products of fractals, Trans. Amer. Math. Soc. 357 (2005), no. 2, 571-615. MR2095624 (2005m:31016)

[S7] R. Strichartz, Differential equations on fractals: a tutorial, Princeton University Press, 2006 (to appear).

[SU] R. Strichartz and M. Usher, Splines on fractals, Math. Proc. Cambridge Phil. Soc. 129 (2000), 331-360. MR1765920 (2001c:28016)

[T1] A. Teplyaev, Spectral analysis on infinite Sierpinski gaskets, J. Functional Anal. 159 (1999), 537-567. MR1658094 (99j:35153)

[T2] A. Teplyaev, Gradients on fractals, J. Functional Anal. 174 (2000), 128-154. MR.1761365 (2001h:31012)

Department of Mathematics, University of Michigan, Ann Arbor, Michigan 481091109

Current address: Division of Applied Mathematics, Brown University, Providence, Rhode Island 02912

E-mail address: Nitsan_Ben-Gal@brown.edu

Department of Mathematics, University of California, Los Angeles, California 90095

Current address: Department of Applied Physics \& Applied Mathematics, Columbia University, New York, New York 10027

E-mail address: ams2134@columbia.edu

Department of Mathematics, Malott Hall, Cornell University, Ithaca, New York $14853-4201$

E-mail address: str@math.cornell.edu

Department of Mathematics, SUny Binghamton, Binghamton, New York 13902-6000

Current address: Department of Physics, SUNY, Stony Brook, New York 11794

E-mail address: cyoung@grad.physics.sunysb.edu 